This document is confidential and is proprietary to the American Chemical Society and its authors. Do not copy or disclose without written permission. If you have received this item in error, notify the sender and delete all copies.

\title{
Experimental and Theoretical Determination of Dissociation Energies of Dispersion-Dominated Aromatic Molecular Complexes
}

\begin{tabular}{|r|l|}
\hline Journal: & Chemical Reviews \\
\hline Manuscript ID & cr-2015-006525.R1 \\
\hline Manuscript Type: & Thematic Review \\
\hline Date Submitted by the Author: & n/a \\
\hline Complete List of Authors: & $\begin{array}{l}\text { Frey, Jann; Universität Bern, Dept. für Chemie und Biochemie } \\
\text { Holzer, Christof; Karlsruhe Institute of Technology, Institute of Physical } \\
\text { Chemistry, Theoretical Chemistry Group } \\
\text { Klopper, Wim; KIT Karlsruhe, Institut Nanotechnology, } \\
\text { Leutwyler, Samuel; Universität Bern, Dept. für Chemie und Biochemie }\end{array}$ \\
\hline
\end{tabular}

\section{SCHOLARONE ${ }^{\text {m }}$}

Manuscripts 


\title{
Experimental and Theoretical Determination of
}

\section{Dissociation Energies of Dispersion-Dominated}

\section{Aromatic Molecular Complexes}

\author{
Jann A. Frey, ${ }^{\dagger}$ Christof Holzer ${ }^{\ddagger}$ Wim Klopper ${ }^{\ddagger}$ and Samuel Leutwyler ${ }^{*} \dagger$ \\ Departement für Chemie und Biochemie, Universität Bern, Freiestrasse 3, CH-3012 Bern, \\ Switzerland, and Institute of Physical Chemistry, Karlsruhe Institute of Technology (KIT), \\ Fritz-Haber-Weg 2, D-76131 Karlsruhe, Germany \\ E-mail: klopper@kit.edu
}

\begin{abstract}
The dissociation energy $\left(D_{0}\right)$ of an isolated and cold molecular complex in the gas-phase is a fundamental measure of the strength of the intermolecular interactions between its constituent moieties. Accurate $D_{0}$ values are important for the understanding of intermolecular bonding, for benchmarking high-level theoretical calculations and for the parametrization of force-field models used in fields ranging from crystallography to biochemistry. We review experimental and theoretical methods for determining gas-phase $D_{0}$ values of $\mathrm{M} \cdot \mathrm{S}$ complexes, where $\mathrm{M}$ is a (hetero)aromatic molecule and $\mathrm{S}$ is a closed-shell "solvent" atom or molecule. The experimental methods discussed involve M-centered $\left(S_{0} \rightarrow S_{1}\right)$ electronic excitation, which is often followed by ionization to the $\mathrm{M}^{+} \cdot \mathrm{S}$ ion. The $D_{0}$ is measured by depositing a defined amount of vibrational energy in the neutral ground state, giving $M^{\neq} \cdot S$, the neutral

\footnotetext{
${ }^{*}$ To whom correspondence should be addressed Germany

†Departement für Chemie und Biochemie, Universität Bern, Freiestrasse 3, CH-3012 Bern, Switzerland

Institute of Physical Chemistry, Karlsruhe Institute of Technology (KIT), Fritz-Haber-Weg 2, D-76131 Karlsruhe,
\end{abstract}


$S_{1}$ excited state, giving $\mathrm{M}^{*} \cdot \mathrm{S}$, or the $\mathrm{M}^{+} \cdot \mathrm{S}$ ion ground state. The experimental methods and their relative advantages and disadvantages are discussed. Based on the electronic structure of $\mathrm{M}$ and S, we classify the M.S complexes as type I, II or III, and discuss characteristic properties of their respective potential-energy surfaces that affect or hinder the determination of $D_{0}$. Current theoretical approaches are reviewed, which comprise methods based on a KohnSham reference determinant as well as wave function-based methods based on coupled-cluster theory. 


\section{Contents}

1 Introduction $\quad 8$

2 Excitation and Ionization of M.S Complexes: A Classification $\quad 11$

2.1 Type I Complexes . . . . . . . . . . . . . . . . . . . . . . . . 12

2.2 Type II Complexes . . . . . . . . . . . . . . . . . . . . . . . . 12

2.3 Type III Complexes $\ldots \ldots \ldots$. . . . . . . . . . . . . . . . . . . . 14

3 Experimental Techniques for the Determination of Dissociation Energies of Dispersion$\begin{array}{ll}\text { dominated Dimers } & 15\end{array}$

3.1 Dispersed Fluorescence Methods (DIS) . . . . . . . . . . . . . . 15

3.2 Two-color Appearance Energies and Ion Breakdown Measurements . . . . . . . . 16

3.3 MATI-based Dissociation Energy Measurements of M·S Ions . . . . . . . . . . . . 17

3.4 Velocity Map Imaging $(\mathrm{VMI}) \ldots \ldots \ldots$

3.5 Stimulated Emission Pumping/Resonant Two-photon Ionization (SEP-R2PI) . . . . 19

4 Quantum Chemical Challenges for Calculating Dispersive Interactions 21

4.1 Coupled-cluster Theory for Large Systems . . . . . . . . . . . . . . . . . . . 22

4.2 Explicitly-correlated Coupled-cluster Theory . . . . . . . . . . . . . . . . 24

4.3 Multilevel Schemes . . . . . . . . . . . . . . . . . . . . 25

4.4 MP2 Complete Basis-set Limit . . . . . . . . . . . . . . . . . . . . 26

4.5 Spin-component-scaled Methods . . . . . . . . . . . . . . . . . 28

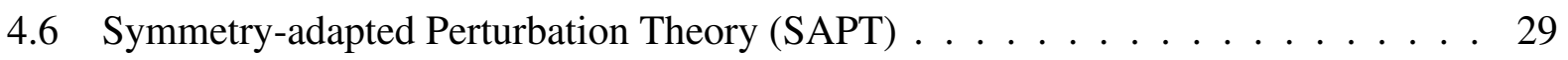

4.7 Dispersion-corrected Density-functional Theory . . . . . . . . . . . . . 30

4.8 Random-phase Approximation (RPA) . . . . . . . . . . . . . . 30

4.9 Quantum Monte Carlo and Semiempirical Methods . . . . . . . . . . . . . . . . 32

4.10 Excited States $\left(S_{1}\right)$ of Weakly Interacting Complexes . . . . . . . . . . . 32

4.11 Zero-point Vibrational Energy . . . . . . . . . . . . . . 33 
5 Examples of Binding and Dissociation Energies of Dispersively Bound Complexes 34

5.1 Type I Complexes: Two Systems with Localized HOMOs. . . . . . . . . . . . . . 34

$5.1 .1 \mathrm{Ar}_{2}, \mathrm{Kr}_{2}, \mathrm{Xe}_{2},\left(\mathrm{~N}_{2}\right)_{2}$ and $(\mathrm{CO})_{2} \ldots \ldots \ldots \ldots$

$5.1 .2\left(\mathrm{H}_{2} \mathrm{O}\right)_{2},\left(\mathrm{D}_{2} \mathrm{O}\right)_{2}, \mathrm{H}_{2} \mathrm{O} \cdot \mathrm{HCl}$ and $\left(\mathrm{NH}_{3}\right)_{2} \ldots \ldots \ldots \ldots$

5.2 Type II Complexes: A System with Delocalized HOMO and a System with a Localized HOMO . . . . . . . . . . . . . . . . . . . . 36

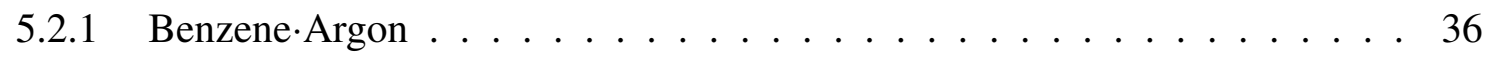

5.2.2 Complexes with Carbazole . . . . . . . . . . . . . . 38

5.3 Type III Complexes: Two Systems with Delocalized HOMOs . . . . . . . . . . . . 39

5.3.1 Benzene Dimer . . . . . . . . . . . . . . . 39

5.3.2 Toluene Dimer . . . . . . . . . . . . . . . . 42

5.3 .3 Indole Benzene . . . . . . . . . . . . . . 43

5.3.4 Complexes with Anisole . . . . . . . . . . . . . . . 45

6 Summary and Outlook 46 


\section{Biographies}

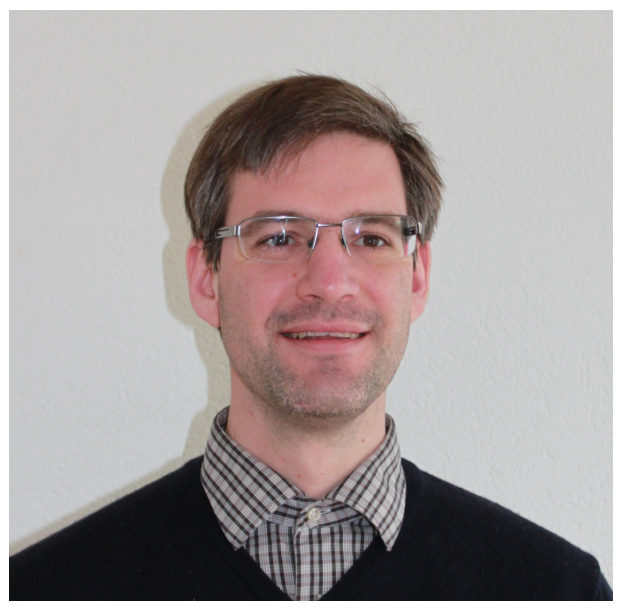

Jann Frey was born in Bern, Switzerland, in 1977. He studied chemistry at the University of Bern where he received his diploma in 2001 and his $\mathrm{Ph}$. D. in 2006. He worked as a postdoctoral researcher in the groups of Prof. John. P. Simons (Univ. of Oxford, 2007) and Prof. Peter Chen (ETH Zürich,2008-2010). He currently teaches chemistry at Gymnasium Liestal and spent a research sabbatical (2014-2015) in the Leutwyler research group. His research interests are in the field of weakly bound complexes.

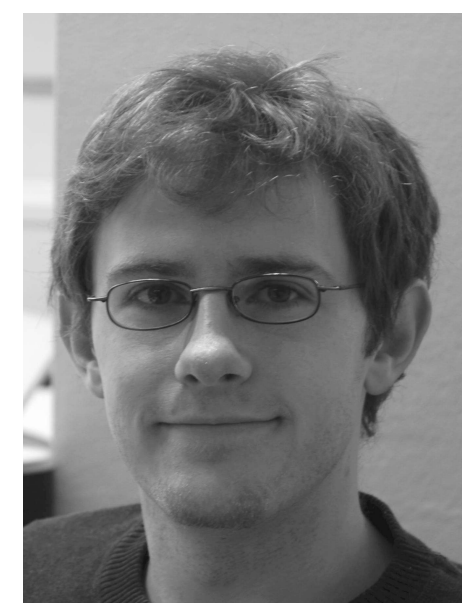

Christof Holzer was born in Graz, Austria, in 1989. He studied chemistry at the Graz University of Technology (2009-2015). He received his MSc. in 2015 from the Graz University of Technology under the supervision of Nadia Mösch-Zanetti. In 2015 he joined the research group of Wim 
Klopper at the Karlsruhe Institute of Technology. His research interests include the development of new (explicitly correlated) methods to improve the description of weakly bonded systems.

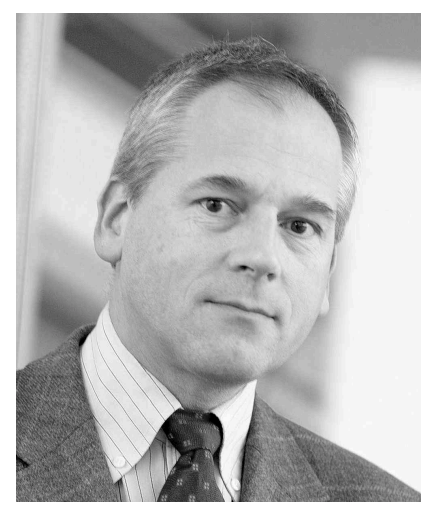

Wim Klopper was born in Opperdoes, The Netherlands, in 1961. He studied chemistry at the University of Leiden (1979-1982) and at the Ruhr-Universität Bochum (1982-1985), where he received his Ph.D. in 1989 under the supervision of Werner Kutzelnigg. He was post-doctoral research associate in the groups of Jan Almlöf (University of Minnesota, 1991) and Hans Peter Lüthi (ETH Zurich, 1993-1996) and he spent three years (1996-1999) at the University of Oslo as Førsteamanuensis (Associate Professor) and three years (1999-2002) at the University of Utrecht as Akademie Onderzoeker of the Royal Netherlands Academy of Arts and Sciences (KNAW). In September 2002, he accepted the position of Professor of Theoretical Chemistry at the Universität Karlsruhe (TH) and in 2003 he became Group Leader at the Institute of Nanotechnology at the Forschungszentrum Karlsruhe (since 2009, the Universität Karlsruhe (TH) and the Forschungszentrum Karlsruhe have merged into the Karlsruhe Institute of Technology). Wim Klopper has received the Venia Legendi for Theoretical Chemistry (ETH Zurich, 1998), the Hans G. A. Hellmann Prize of the Arbeitsgemeinschaft Theoretische Chemie (1999), and the Medal of the International Academy of Quantum Molecular Science (1999). He became Member of the International Academy of Quantum Molecular Science in 2011. His research interests include the further development of explicitly correlated electronic structure methods and the accurate computation of molecular energies as well as properties. 


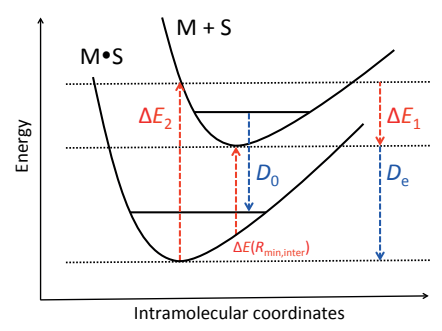

Figure 1: TOC Figure

Samuel Leutwyler was born in Switzerland in 1952. He studied chemistry at the University of Bern and received his Ph.D. in 1979 under the supervision of Ernst Schumacher. He was post-doctoral research associate in the research group of Uzi Even and Joshua Jortner (Tel-Aviv University, 1979-1980). He spent four years (1980-1984) at the University of Basel in the groups of J. P. Maier and E. Heilbronner, obtaining a venia legendi (Habilitation) in Physical Chemistry in 1984. He then moved to the University of Bern, where he is Professor of Physical Chemistry since 1990. He was awarded the Nernst-Haber-Bodenstein-Preis of the Deutsche Bunsen-Gesellschaft (1986), the Ruzicka-Preis für Chemie (1989) of the Eidg. Technische Hochschulen (ETH Schulrat) and the Robert-Bunsen Vorlesung of the Deutsche Bunsengesellschaft (2015). His research interests include UV and IR molecular-beam laser spectroscopy of nucleic acid base pair analogues and of van der Waals and H-bonded complexes and clusters, photoinduced proton and hydrogen atom transfer in clusters and high-resolution ultrafast rotational coherence spectroscopy. 


\section{Introduction}

Noncovalent interactions between molecules are pivotal for all chemical and biochemical processes, because the mutual approach of two neutral molecules at distances significantly larger than $\approx 4 \AA$ is driven by intermolecular interactions that extend over medium- to long-range intermolecular distances. Noncovalent interactions are at the heart of molecular recognition, ${ }^{1}$ and important areas of research like supramolecular chemistry, crystal engineering, biochemical signal transduction or selective catalysis are governed by such processes. A quantitative understanding of intermolecular interactions therefore serves as a basis for the understanding of these complex fields.

The ground state dissociation energy $D_{0}$ and well depth $D_{e}$ are among the most important parameters characterizing intermolecular interactions and the associated intermolecular potential energy surfaces (PESs). However, even today, accurate experimental gas-phase dissociation energies are only known for a very limited number of dispersively bound molecular dimers and hetero-dimers of chemical interest. ${ }^{2}$

Already in 1930, London identified three types of intermolecular interactions: ${ }^{3}$ (1) Electrostatic interactions between two dipolar (or in general multipolar) molecules. (2) Inductive interactions that arise when a dipolar (or quadrupolar) molecule induces a dipole or quadrupole moment in a nearby molecule. Since the induced moments are always oriented in such a way as to give a stabilizing interaction with the inducing molecule, inductive interactions are always binding. (3) The dispersive interaction, which - in a semi-classical picture - arises from very rapid temporal fluctuations of the electrons in the charge distribution around a closed-shell atom or molecule $A$ that induce dipolar (or higher multipolar) charge motions in a neighboring atom or molecule $B$, and vice versa from $B$ on $A$.

Non-covalently bonded systems are usually classified according to the dominating attractive interactions in their minimum-energy region into hydrogen-bonded (H-bonded) and dispersion-bonded (or van der Waals) (vdW) complexes. However, not all intermolecular complexes are covered by this classification (such as $\mathrm{NH}_{3} \cdots \mathrm{Cl}_{2}$ ), and demarcations between these groups are not always 
sharp. H-bonded complexes are dominated by electrostatic interactions between well-defined Hdonor and acceptor atoms. The potential minima are well defined with steep walls that are typically separated by high interconversion barriers. In contrast, the potential minima of dispersion-bonded complexes are shallower and less well separated, so experiments often need to consider a number of isomers, and theory needs to characterize not only multiple minima but also the barriers between them, as well as the zero-point vibrational energy (ZPVE) of the intermolecular vibrations. The ZPVE can be larger than the barriers between the minima, leading to large-amplitude zero-point motions. Second, for H-bonded complexes the electrostatic contribution to the interaction energy usually converges with affordable computational efforts (i.e., MP2 at the complete basis set limit), while the dispersion terms require higher order (e.g., $\operatorname{CCSD}(\mathrm{T}))$ calculations. Any uncertainties introduced by approximative treatments of the dispersive contribution — be it by corrected density functionals (DFT-D) or by adding $\Delta(\mathrm{CCSD}(\mathrm{T})-\mathrm{MP} 2)$ correction values evaluated in small basis sets - only affect a fraction of the total interaction energy of H-bonded systems, but a major part in dispersion-bonded complexes. Third, the dissociation energy of dispersion-bonded complexes is typically smaller than that of hydrogen-bonded dimers.

The research on non-covalently bonded gas-phase complexes has been summarized in several reviews. Most reviews have focused on H-bonded systems. ${ }^{4-7}$ Exceptions are the reviews of Topp (1993), who reports on structures and vibrational predissociation dynamics of complexes of small molecules with chromophores such as perylene or 2,5-diphenylfuran determined by fluorescence spectroscopy, ${ }^{8}$ and Kendler and Haas (1997), ${ }^{9}$ discussing the binding energies of vdW complexes with Ar. In 2015, Hobza and co-workers published a thorough comparison study between theoretical and experimental dissociation energies of 11 hydrogen bonded and 11 dispersive bonded complexes, finding an average agreement of $6 \% .{ }^{10}$

Below, we review experimental and theoretical approaches to determining the dissociation energies of dispersion-bonded M.S complexes that consist of an aromatic chromophore, denoted $\mathrm{M}$, and a "solvent" atom or molecule, denoted S. When comparing calculated and experimental interactions, it is important to note the (subtle) differences between various definitions of the energies. These 
definitions are illustrated in Figure 2 and the particular energies are the following:

(1) The intermolecular interaction energy $\Delta E\left(R_{\text {min,inter+intra }}\right)$, which corresponds to the energy difference between the fully optimized complex (or dimer) and the $\mathrm{M}$ and $\mathrm{S}$ monomers in the structures that they adopt in the complex. This energy contribution is also referred to as two-body energy $\Delta E_{2}$. This energy does not include the deformation energy $\Delta E_{\mathrm{def}}$ of the monomers, which comprises the energy differences between the monomers in their isolated, relaxed geometries and their structures in the dimer. The latter contribution is necessarily positive and is also denoted the one-body energy $\Delta E_{1}$. Furthermore, the interaction energy does not take into account the ZPVE of the complex, nor the changes of the monomer ZPVEs upon dimerization. Although the interaction energy is not experimentally accessible, it is valuable, since it is a direct measure of the stabilization arising from the interaction of the $\mathrm{M}$ and $\mathrm{S}$ wave functions. A further decomposition of the interaction energy into electrostatic, dispersive, inductive and repulsive exchange interactions can be performed by symmetry-adapted perturbation-theory (SAPT). Here the M-S geometry is first fully optimized using a long-range corrected DFT method or correlated quantum-chemical method. The SAPT interaction energy $\Delta E_{\mathrm{SAPT}}$ and its contributions are calculated at this geometry using a high-quality perturbation-theoretical method. Note that $\Delta E_{\mathrm{SAPT}}$ needs to be corrected by the monomer deformation energies and the ZPVE of the complex and monomers in order to be comparable with experiment.

(2) The intermolecular electronic binding energy $D_{e}\left(R_{\text {min,inter+intra }}\right)$, which is evaluated relative to the energies of $\mathrm{M}$ and $\mathrm{S}$ in their optimized monomer structures, $D_{e}=-\Delta E_{1}-\Delta E_{2}$. Note that $D_{e}\left(R_{\text {min,inter+intra }}\right)$ is smaller in magnitude than $\Delta E\left(R_{\text {min,inter+intra }}\right)$ since it contains the (always positive) deformation energy $E_{\text {def. }}$. For weakly bound dimers, the deformation of $\mathrm{M}$ and $\mathrm{S}$ may be negligible, leading to deformation energies $<0.5 \mathrm{~kJ} / \mathrm{mol}$, but $E_{\mathrm{def}}$ increases for larger $D_{e}$ values. For example, $E_{\mathrm{def}}$ values of more than $10 \%$ of $D_{e}$ have been reported for H-bonded nucleobase dimers. ${ }^{11}$ Because $D_{e}\left(R_{\text {min,inter+intra }}\right)$ does not contain any ZPVE contributions, it usually overestimates the intermolecular interaction strength (cf. Figure 2) and is also not directly measurable. However, it corresponds to the minimum of the $3 N-6$-dimensional PES of the complex. 
(3) The intermolecular dissociation energy $D_{0}\left(R_{\text {min,inter+intra }}\right)$. Here the vibrational zero-point energies of all the intramolecular and intermolecular vibrations of the complex, $\mathrm{ZPVE}_{\mathrm{tot}}\left(R_{\text {min,inter+intra }}\right)$, as well as the ZPVE of M and S need to be calculated separately at the respective geometries $R_{\min , \text { inter+intra }}, R_{\min , \mathrm{M}}$ and $R_{\min , \mathrm{S}}$. The zero-point energy change $\Delta \mathrm{ZPVE}=$ $\mathrm{ZPVE}_{\mathrm{tot}}-\mathrm{ZPVE} \mathrm{M}_{\mathrm{M}}-\mathrm{ZPVE}_{\mathrm{S}}$ is subtracted from $D_{e}$ to give the dissociation energy $D_{0}$ of the complex. In our view, this is the theoretical quantity that should be compared to experimental $D_{0}$ values. Note that the ZPVE of M.S contains the intermolecular ZPVE of the six intermolecular vibrational coordinates plus the change of the intramolecular $3 N_{\mathrm{M}}-6$ and $3 N_{\mathrm{S}}-6$ ZPVEs of the M and S moieties. The latter are often a large (and unrecognized!) contribution to $\triangle \mathrm{ZPVE}$, and may outweigh the intermolecular ZPVE contribution. The change of intramolecular ZPVE is especially important in $\mathrm{H}$-bonded complexes, because the ZPVE of the $\mathrm{X}-\mathrm{H}$ bond is large and in most cases reduced by $\mathrm{H}$-bond formation.

(4) The fixed-fragments interaction energy $\Delta E\left(R_{\text {min,inter }}\right)$ for a complex or dimer that is geometryoptimized with respect to its intermolecular but not its intramolecular coordinates. This amounts to the hypothetical interaction energy between two non-deformed monomers in their respective monomer geometries. $\Delta E\left(R_{\text {min,inter }}\right)$ is mostly evaluated for the construction of intermolecular potential energy surfaces. Even for small molecules, the large number of points needed to accurately probe the full six-dimensional intermolecular fixed-fragments interaction-energy surface precludes a geometry relaxation of the complex at every point.

\section{Excitation and Ionization of M.S Complexes: A Classifica- tion}

It is useful to divide M.S complexes into the following three types, which depend on the electronic structure of $\mathrm{M}$ and $\mathrm{S}$ : 


\subsection{Type I Complexes}

Neither M nor S possesses delocalized $\pi$-electrons. While this property excludes aromatic systems - the focus group of this review - we include Type I complexes because the same experimental techniques can be employed for the determination of their dissociation energies as for Type II and III complexes discussed below. Some of the methodological advantages and drawbacks are better documented for Type I complexes than for the other two groups.

Assuming that the adiabatic ionization energy (AIE) of $\mathrm{M}$ is lower than that of $\mathrm{S}$, ionization typically occurs by removal of an electron from a non-bonding $n$-orbital (e.g., $\left.n_{\mathrm{N}}, n_{\mathrm{O}}, n_{\mathrm{S}}\right) \mathrm{HOMO}$ of M, leaving a localized "hole" on the heteroatom. This leads to large geometry changes between $\mathrm{M} \cdot \mathrm{S}$ and $\mathrm{M}^{+} \cdot \mathrm{S}$. In terms of vibronic spectroscopy, the Franck-Condon factors (squares of vibrational overlap integrals) between the neutral and ionic ground states become very small. Experimental schemes for determining $D_{0}$ that rely on thermochemical cycles involving ionization and the ion ground state $D_{0}^{+}$, see Figure 3, can therefore be problematic. They are not recommended for type-I and III complexes (see below).

\subsection{Type II Complexes}

Only M has delocalized $\pi$-electrons. The lowest excited singlet state $S_{1}$, denoted $\mathrm{M}^{*}$, is usually a ${ }^{1} \pi \pi^{*}$ excitation that lies in the visible or near-UV region. Solvent molecules $\mathrm{S}$ whose frontier orbitals are $\sigma$ - or non-bonding orbitals have high-lying (far-UV) electronic excitations corresponding to $\sigma \rightarrow \sigma^{*}, n \rightarrow \sigma^{*}$ or Rydberg excitations. For this reason, many common solvents $\mathrm{S}$ are transparent down to $\approx 200 \mathrm{~nm}$. In this case, the $S_{0} \rightarrow S_{1}$ excitation of M.S (M*S) remains M-centered, and since the $\pi$-electron system is delocalized, the electron density change at each atom is quite small. Therefore, the M.S geometry, vibrational frequencies and dissociation energy undergo small changes upon ${ }^{1} \pi \pi^{*}$ excitation, as is schematically shown in Figure 4. With regard to ionization, the electron is mainly removed from the $\pi$-HOMO of $\mathrm{M}$ if the AIE of $\mathrm{M}$ is lower than that of S, and analogous considerations apply for the $\mathrm{M}^{+} \cdot \mathrm{S}$ ion ground state. In general, the electronic excitation energies change only moderately when an aromatic $\mathrm{M}$ is complexed with a non-aromatic closed- 
shell S molecule. The small geometry changes ensure good overlaps between the initial and final vibronic wave functions (i.e., large Franck-Condon factors) in excitation. This opens the door to the investigation of the properties of $\mathrm{M} \cdot \mathrm{S}$ and $\mathrm{M}^{*} \cdot \mathrm{S}$ using the toolbox of visible/UV tunable laser experiments that have been developed over the last forty years.

Care must however been taken when investigating the $\mathrm{M}^{+} \cdot \mathrm{S}$ state of such complexes when $\mathrm{S}$ is not a noble gas atom: upon ionization, the charge on $\mathrm{M}^{+}$leads to an additional charge-multipole interaction with S (if S is polar or quadrupolar) and in every case to charge-induced multipole interactions. These may lead to large geometry changes between the neutral and ion ground states. Well known examples are the neutral complexes whose geometries are dominated by $\mathrm{X}-\mathrm{H} \cdots \pi$ nonclassical hydrogen-bonding interactions, as in the $\mathrm{M} \cdot \mathrm{HF}, \mathrm{M} \cdot \mathrm{HCl}, \mathrm{M} \cdot \mathrm{H}_{2} \mathrm{O}$ and $\mathrm{M} \cdot$ methanol complexes. Upon ionization the charge on $\mathrm{M}^{+}$renders the $\mathrm{X}-\mathrm{H} \cdots \pi$ hydrogen bond repulsive, the positive $\mathrm{H}$ atom of the $\mathrm{H}-\mathrm{X}$ bond is repelled and the stable ion ground state minimum has a completely different geometry. This creates several problems for the analysis: (1) The vertical ionization (i.e., at the neutral ground-state geometry) is highly efficient, but this can lead to energies close to or above the dissociation energy $D_{0}$ of the $\mathrm{M}^{+} \cdot S$ ion ground state, leading to direct dissociation or vibrational predissociation (VP). (2) Even if vertical ionization leads to an energy below $D_{0}$, it may be difficult or impossible to experimentally access the ion ground state minimum (i.e., the adiabatic ionization energy), so that the AIE(M.S) cannot be measured. (3) Due to the larger $D_{0}$ in the ion combined with the geometry change, the nascent $\mathrm{M}^{+}$and $\mathrm{S}$ fragments arising from the $\mathrm{M}^{+} \cdot \mathrm{S}$ dissociation may be vibrationally and rotationally hotter than those arising from dissociation on the ground-state surface. It also takes longer for the internal energy in the ion ground state to randomize before VP, giving rise to a wide distribution of time delays ( $1-10,000 \mathrm{~ns})$ between the ionization of M.S and the instant of its decay. ${ }^{12}$ The dissociation threshold can then only be obtained using unimolecular dissociation models for the kinetic energy release (KER). These involve extensive theoretical modeling and calculations and are subject to uncertainties that are not small and often hard to quantify. ${ }^{12}$ 


\subsection{Type III Complexes}

Both $\mathrm{M}$ and $\mathrm{S}$ have delocalized $\pi$-electron systems. This interaction type occurs in many dimers of chemical and biochemical interest (e.g., $\pi$-stacked aromatics, DNA bases, charge-transfer complexes, etc.), which are therefore widely studied. Two common geometry types exist, denoted T-shaped and $\pi$-stacked (with variations such as slipped-stacked). Since the AIEs of M and S are closer together (or identical for self-dimers $\mathrm{M}_{2}$ ) than for type II complexes, the ionization must be viewed as removal of electronic charge from the HOMOs of both $\mathrm{M}$ and $\mathrm{S}$. The ion ground state involves stabilizing charge-resonance contributions of the type $\mathrm{M}^{+} \cdot \mathrm{S} \leftrightarrow \mathrm{M} \cdot \mathrm{S}^{+}$, which are strongest for symmetric self-dimers $(\mathrm{M} \cdot \mathrm{M})^{+} .{ }^{13}$ Charge-resonance stabilization also remains important in heterodimer ions. The most stable ionic geometry is typically a $\pi$-stacked structure which maximizes the charge-resonance interactions. If the neutral structure is T-shaped, the geometry changes completely upon ionization; if the neutral is $\pi$-stacked, the M.S interplanar distance contracts strongly upon ionization. In both cases the large differences between the neutral and ionic geometries result in small Franck-Condon factors in ionization. These considerations show that considerable problems arise when trying to determine the experimental dissociation energies of type I and III complexes using ionizative methods.

The review is organized as followed: In Section 3 we review and describe experimental techniques for determining ground state dissociation energies $D_{0}\left(S_{0}\right)$ of $\mathrm{M} \cdot \mathrm{S}$ van der Waals complexes. Section 4 summarizes the challenges of a quantum chemical calculation of dissociation energies. In Section 5 we present prominent examples of experimentally and theoretically assessed dissociation energies of vdW complexes. 


\section{Experimental Techniques for the Determination of Dissocia- tion Energies of Dispersion-dominated Dimers}

There has been work on the measurement of $\mathrm{M} \cdot \mathrm{S}$ dissociation energies, $D_{0}(\mathrm{M} \cdot \mathrm{S})$, since the invention of supersonic-jet spectroscopy. This involves (1) producing the M.S complexes in the supersonic-jet expansion, (2) cooling them to their lowest $\left(v^{\prime \prime}=0\right)$ vibrational level, and (3) performing different types of vibronic spectroscopic and/or photoionization measurements. One-step photoionization techniques typically involve a vacuum-ultraviolet (VUV) laser, a VUV lamp or a synchrotron light source (the latter two being monochromatized with a VUV monochromator), generating light in the $7-12 \mathrm{eV}$ range. The two-step measurements typically involve two tunable UV lasers, where the first laser resonantly excites the M moiety of M.S and the second laser photoionizes the excited $\mathrm{M}^{*} \cdot \mathrm{S}$ complex. By suitably combining vibronic or vibrational excitation frequencies and photoionization thresholds of $\mathrm{M}, \mathrm{S}$ and $\mathrm{M} \cdot \mathrm{S}$ and applying thermochemical cycles, as shown in Figure 5, the dissociation energies $D_{0}\left(\mathrm{M}^{+} \cdot \mathrm{S}\right)$ and $D_{0}(\mathrm{M} \cdot \mathrm{X})$ can be determined. Alternatively, vibrationally excited $\mathrm{M} \cdot \mathrm{S}$ complexes, denoted $\mathbf{M}^{\neq} \cdot \mathrm{S}$, can be populated via infrared (IR) absorption, by stimulated Raman pumping or by vibronic pump-and-dump (stimulated-emissionpumping $=\mathrm{SEP})$ techniques. These methods allow to directly determine $D_{0}(\mathrm{M} \cdot \mathrm{X})$ without passing through the ion ground state.

\subsection{Dispersed Fluorescence Methods (DIS)}

This method was pioneered by Levy and co-workers in the early 1980 s to determine the dissociation energies of $\mathrm{I}_{2} \cdot \mathrm{X}(\mathrm{X}=\mathrm{Ne}, \mathrm{Ar}, \mathrm{Kr}, \mathrm{Xe}) \mathrm{vdW}$ complexes. ${ }^{14}$ A series of dispersed fluorescence spectra of the $\mathrm{I}_{2} \cdot \mathrm{X}$ complex are recorded while exciting the $\tilde{X} \rightarrow \tilde{B}$ electronic origin $\left(0_{0}^{0}\right)$ band of the $\mathrm{I}_{2}$ moiety to increasing vibrational quantum number $v^{\prime}$ of the $\tilde{B}$ excited state. If the energy of the $\tilde{B} ; v^{\prime}$ level exceeds the dissociation energy $D_{0}^{\prime}(\tilde{B})$, the $\mathrm{I}_{2}^{*} \cdot \mathrm{X}$ complex undergoes excited state VP, i.e., $I_{2}^{*} \cdot X \rightarrow I_{2}^{*}+X$. If the VP rate exceeds the luminescence rate of the $\tilde{B}$ state, the emission from bare $\mathrm{I}_{2}^{*}$ is observed which can be spectrally discriminated from that of $\mathrm{I}_{2}^{*} \cdot \mathrm{X}$. The lowest vibronic 
level $v^{\prime}$ of $\mathrm{I}_{2}^{*} \cdot \mathrm{X}$ that predissociates to bare $\mathrm{I}_{2}^{*}$ provides an upper limit of $D_{0}^{\prime}$. A corresponding upper limit to the ground-state dissociation energy $D_{0}^{\prime \prime}$ is obtained from $D_{0}^{\prime}$ from the spectral shift $\delta v=D_{0}^{\prime}-D_{0}^{\prime \prime}$ of the $0_{0}^{0}$ band origins of $\mathrm{I}_{2}$ and of $\mathrm{I}_{2} \cdot \mathrm{X}$. A schematic illustration of the method is given in Figure 6.

This method has been extended to $\mathrm{M} \cdot \mathrm{X}$ complexes such as s-tetrazine $\cdot A r$, bracketing $D_{0}$ between $3.0-4.3 \mathrm{~kJ} / \mathrm{mol}){ }^{15}$ An important limit to the DIS method is the existence of excited states above the dissociation limit with lifetimes larger than the experimental time-frame, as it has been observed for example for the benzene.argon complex. ${ }^{16}$

The method requires the emission of $\mathbf{M}^{*} \cdot \mathrm{S}$ to differ sufficiently from that of $\mathrm{M}^{*}$, such that the two spectra can be unambiguously separated. Note that emission from the highly excited complex can be shifted to the same region as that of the monomer dissociation product, which can lead to dissociation being incorrectly deduced as having occurred. ${ }^{17}$ For larger systems or for complexes with large dissociation energies, the spectra of both $\mathrm{M}^{*}$ and $\mathrm{M}^{*} \cdot \mathrm{S}$ become more complicated, which often leads to overlapping emission spectra. To overcome this limitation, a two-laser scheme has been introduced by Wittmeyer and Topp ${ }^{18}$ in which the first laser vibronically excites the investigated complex. If enough energy for VP in the excited state is deposited into the complex, the formation of vibrationally excited $\mathbf{M}^{\neq}$ground state fragments is expected. These hot fragments are probed by a second laser which is tuned to a hot band and fired $\approx 100$ ns after the excitation laser. $^{18}$

\subsection{Two-color Appearance Energies and Ion Breakdown Measurements}

This technique involves pumping the $S_{0} \rightarrow S_{1} 0_{0}^{0}$ band of $\mathrm{M} \cdot \mathrm{S}$, after which the photoionization efficiency (PIE) curve of the excited $\mathrm{M}^{*} \cdot \mathrm{S}$ state is recorded up to energies that are high enough for VP to occur in the $\mathrm{M}^{+} \cdot \mathrm{S}$ ion ground state. At the VP threshold the $\mathrm{M}^{+} \cdot \mathrm{S}$ ion signal disappears and the signal of the daughter $\mathrm{M}^{+}$ion appears; both ion signals are simultaneously monitored. A schematic illustration is given in Figure 7. Since ionization produces both $\mathrm{M}^{+} \cdot \mathrm{S}$ ions and photoelectrons and the energy carried away by the photoelectron is not measured in appearance energy measurements, 
this unknown partition of energy between $\mathrm{M}^{+}, \mathrm{S}$ and the electron leads to a considerable indeterminacy. In practice, the VP thresholds in both the $\mathrm{M}^{+} \cdot \mathrm{S}$ and $\mathrm{M}^{+}$ion signals are often unclear, and both are extrapolated to the same VP threshold. Since 2007, Mikami and co-workers have applied this technique to determine the $D_{0}\left(\mathrm{M}^{+} \cdot \mathrm{S}\right)$ of $\mathrm{CH} \cdots \pi$ bonded complexes such as benzene.ethene, benzene acetylene, benzene $\cdot \mathrm{CH}_{2} \mathrm{Cl}_{2}$ and benzene $\cdot \mathrm{CHCl}_{3} \cdot{ }^{19-23}$

To discern between stable and metastable $\mathrm{M}^{+} \cdot \mathrm{S}$ ions, Neusser and co-workers have developed a method to determine the appearance energies of $\mathrm{M}^{+}$ions, using a reflectron time-of-flight mass spectrometer (RETOF). ${ }^{24}$ The metastable ions that decay in the drift-region of the RETOF have a different kinetic energy than the stable ions. The voltages of the reflector are now adjusted in a way that the flight times of the ions are not corrected for the differences in kinetic energy. $\mathrm{M}^{+}$ daughter ions produced by VP in the drift tube appear between the peaks of the stable ions. The appearance energy is determined from the breakdown of the metastable ion signals upon reducing the ionization energy. Breakdown measurements (BM) have been used to determine the dissociation energy of the benzene dimer ${ }^{24}$ as well as the homo- and heterodimers of benzene, toluene and para-difluorobenzenes. ${ }^{25}$

\subsection{MATI-based Dissociation Energy Measurements of M.S Ions}

The mass-analyzed threshold ionization (MATI) method is a photoionization mass-spectrometric technique that allows to accurately measure the excess vibrational energy $E_{\text {exc }}$ that is deposited in the ground state of a molecular ion $\mathrm{M}^{+}$. This is done by tuning the photoionization light source (laser or VUV photon source) and simultaneously measuring the production of the photoions that are produced simultaneously to threshold (or zero-kinetic energy=ZEKE) photoelectrons. In the MATI technique only the ions are recorded. In a more evolved (but less sensitive) variant, the threshold photoelectrons and photoions are recorded in coincidence (TPEPICO). When measuring ions coincidentally with ZEKE photoelectrons, the vibrational energy $E_{\text {exc }}$ of the $\mathrm{M}^{+} \cdot \mathrm{S}$ ion is given by the photon energy minus the adiabatic ionization energy (AIE) of M, AIE(M), see Figure 5 .

Neusser and coworkers pioneered an application of MATI for measuring the dissociation energy 
$D_{0}\left(\mathrm{M}^{+} \cdot \mathrm{S}\right)$, by measuring (1) the AIE of $\mathrm{M} \cdot \mathrm{S}$ and (2) the dissociation threshold of the $\mathrm{M}^{+} \cdot \mathrm{S}$ ion ground state, $D_{0}\left(\mathrm{M}^{+} \cdot \mathrm{S}\right){ }^{26-29}$

The ionization frequency is increased, thereby depositing increasing vibrational energy in the $\mathrm{M}^{+}$centered vibrational ladder of the $\mathrm{M}^{+} \cdot \mathrm{S}$ ion. At (or closely above) the dissociation threshold of $\mathrm{M}^{+} \cdot \mathrm{S}$, the $\mathrm{M}^{+} \cdot \mathrm{S} \rightsquigarrow \mathrm{M}^{+}+\mathrm{S}$ process leads to a breakoff of the $\mathrm{M}^{+} \cdot \mathrm{S}$ ion signal and - correlated with the breakoff - the appearance of the $\mathrm{M}^{+}$daughter ion signal; both mother and daughter ions are simultaneously monitored in the mass spectrometer. Since MATI selects for those ions that appear together with ZEKE (near-threshold) photoelectrons, the breakoff energy/appearance energy is much better defined than in the appearance energy measurements (Section 3.2).

Combining $D_{0}\left(\mathrm{M}^{+} \cdot \mathrm{S}\right)$ with the separately measured adiabatic ionization energies $\mathrm{AIE}\left(\mathrm{M}^{+}\right)$and $\operatorname{AIE}\left(\mathrm{M}^{+} \cdot \mathrm{S}\right)$ yields the dissociation energy $D_{0}(\mathrm{M} \cdot \mathrm{S})$ via the thermochemical cycle shown in Figure 5. ${ }^{6,26,27,30,31}$ Alternatively, the neutral $D_{0}(\mathrm{M} \cdot \mathrm{S})$ can be determined by subtracting AIE(M) from $\mathrm{AE}\left(\mathrm{M}^{+}\right)$, see also Figure 5. ${ }^{32}$ Neusser and co-workers have also determined dissociation energies of hydrogen-bonded and $\pi$-hydrogen bonded complexes of aromatic molecules with an accuracy of $0.1-0.6 \mathrm{~kJ} / \mathrm{mol}^{6,29,33}$

\subsection{Velocity Map Imaging (VMI)}

Since 2000, the groups of Lawrance ${ }^{16,17,34,35}$ and Becucci ${ }^{36}$ have applied the velocity map imaging (VMI) technique to determine the $D_{0}$ values of aromatic $\mathrm{M} \cdot \mathrm{S}$ complexes and self-dimers $\mathrm{M}_{2}$. VMI is an enhanced resolution variant of ion imaging, for a detailed description, see ref. 16. The VMI technique is also discussed in Section 5.1.2 in the context of dissociation energy determination of H-bonded dimers (type I complexes).

The M.S dissociation energy is measured (a) in the $S_{1}$ state or (b) in the ion ground state. VMI schemes of both variants (a,b) are shown in Figure 8. In Figure 8(a), the M.S complex is excited into an $S_{1}$ state vibronic level that lies slightly above $D_{0}\left(S_{1}\right)$, leading to $S_{1}$ state vibrational predissociation $\mathrm{M}^{*} \cdot \mathrm{S} \rightarrow \mathrm{M}^{*}+\mathrm{S}$. The excited chromophore $\mathrm{M}^{*}$ is then ionized by a second laser. In case (b), the excitation laser pumps M.S into the $v^{\prime}=0$ level of $S_{1}$. The ionization laser is tuned to an 
energy above $D_{0}$ of the $\mathrm{M}^{+} \cdot \mathrm{S}$ ion ground state, leading to VP in the ion ground state, $\mathrm{M}^{+} \cdot \mathrm{S} \rightarrow \mathrm{M}^{+}$ $+\mathrm{S}$. In both cases, the translational recoil energy of the $\mathrm{M}^{+}$fragment is recorded using VMI. The sharing of the translational recoil energy between the detected $\mathrm{M}^{+}$and non-detected $\mathrm{S}$ fragments is taken into account. However, the rotational energy deposited in the M and S fragments is not taken into account (for $\mathrm{S}=\mathrm{X}=\mathrm{Ar}, \mathrm{Kr}$ there is no rotational energy). The maximum translational energy $E_{\text {trans }}(\max )$ measured by VMI is assumed to be the excess energy $E_{\text {excess }}$ that is deposited into the complex by the dissociation laser. The dissociation energy of the investigated state corresponds to $D_{0}=E_{\mathrm{vib}}-E_{\text {trans }}(\max ) .{ }^{16}$ From the spectral shift of the $S_{0} \rightarrow S_{1} 0_{0}^{0}$ band (for a) or the AIE (for b) the ground-state dissociation energy $D_{0}\left(S_{0}\right)$ is obtained via thermochemical cycles. The Lawrance group has studied para-difluorobenzene ( $p$-DFB) with solvents $\mathrm{X}=\mathrm{Ar}, \mathrm{Kr}$ and $\mathrm{H}_{2} \mathrm{O} .{ }^{16,17,34,35} \mathrm{Be}-$ cucci and co-workers have applied VMI to the anisole dimer. ${ }^{36}$ (Anisole) $)_{2}$ is a type-III complex which is expected to undergo large geometry changes after $S_{0} \rightarrow S_{1}$ excitation; indeed the $D_{0}$ measured by VMI ${ }^{36}$ has recently been questioned and revised, based on calculations. ${ }^{37}$ In ref. 37 , the possible existence of a low-lying excited state of the dimer ion is discussed, which could act as a sink for the internal energy. The discrepancy between the $D_{0}$ values reported in refs. 36 and 37 might be related to the existence of different dissociation channels, where the favored channel does not produce the ion fragment in its ground state. As Tables 1 and 2 show, the VMI method has so far delivered very accurate dissociation energies, with errors in the range of $0.04-0.1 \mathrm{~kJ} / \mathrm{mol}$.

\subsection{Stimulated Emission Pumping/Resonant Two-photon Ionization (SEP- R2PI)}

Starting in 1994, the Leutwyler group developed the stimulated-emission pumping/resonant twophoton ionization (SEP-R2PI) method for measuring ground-state $D_{0}$ values of type II M-S complexes. ${ }^{38-41}$ Similar to the ionization-based appearance potential, ion breakdown, MATI and VMI methods described in Sections 3.2 - 3.4, the method is based on the detection of the $\mathrm{M}^{+} \cdot \mathrm{S}$ ion and is mass- (and isotope-) specific. In contrast to the other ionization-based methods, the SEPR2PI method involves excitation of $S_{0}$ state vibrational levels followed by VP on the ground-state 
potential-energy surface. This avoids the problems that arise from large geometry changes of M.S between the $S_{0}$ and lowest ion states.

The SEP-R2PI method involves vibronic pump and “dump" steps $S_{0} \rightarrow S_{1} \rightarrow S_{0}^{\neq}$akin to stimulatedemission pumping (SEP), ${ }^{42}$ which produces vibrationally excited ground-state $\mathbf{M}^{\neq} \cdot \mathrm{S}$, followed by detection of either vibrationally excited $\mathrm{M}^{\neq} \cdot \mathrm{S}$ or the $\mathrm{M}$ fragment, using one-color resonant-twophoton ionization (1C-R2PI). The detection occurs after a time delay $\Delta t$ that is long enough for $\mathrm{M}^{\neq} \cdot \mathrm{S}$ to undergo intramolecular vibrational redistribution and $\mathrm{VP}(\Delta t=0.1-10 \mu \mathrm{s}) .{ }^{39-41}$ The pump, dump and probe steps are induced by three independently tunable UV lasers, as shown in Figure 4. Laser (1) pumps the jet-cooled M.S at the $S_{0} \rightarrow S_{1}$ electronic origin; laser (1) also ionizes a small fraction of the excited molecules, see Figure 3, providing a convenient monitor signal. Laser (2) stimulates "dump" transitions from the $S_{1} ; v^{\prime}=0$ state back down to a vibrationally excited $v^{\prime \prime}>0$ level of the $S_{0}$ state. Because of the high density of the M-S intermolecular vibrations, rapid coupling among the vibrations ensues, leading to intramolecular vibrational energy redistribution (IVR, wiggly arrows in Figure 4). If laser (2) dumps population into vibrational levels lying above $D_{0}\left(S_{0}\right)$, see arrow (2') in Figure 4, the metastable $\mathbf{M}^{\neq} \cdot \mathrm{S}$ complex undergoes VP. After a delay of up to $10 \mu \mathrm{s}$, which allows VP to go to completion, the presence or absence of vibrationally excited $S_{0}^{\neq}$state complexes is probed by laser (3).

In origin-probed dump spectroscopy, both the pump laser (1) and probe laser (3') are tuned to the $0_{0}^{0}$ band of the M.S complex. Scanning the dump frequency (2) yields a signal decrease at every resonant dump transition to the $S_{0}$ state, irrespective of whether the ground-state vibrational level is above or below $D_{0}\left(S_{0}\right)$. (ii) In hot-band probed dump spectroscopy the probe laser (3) is tuned away from the $0_{0}^{0}$ band to hot-band or sequence-band regions, thereby only probing vibrationally hot levels. A signal increase occurs at every resonant dump transition to the $S_{0}$ state, until vibrational levels $v^{\prime \prime}$ are reached that lie above $D_{0}\left(S_{0}\right)$; at this energy the M.S complex vibrationally predissociates and no more bands are observed.

$D_{0}\left(S_{0}\right)$ is thereby bracketed between the highest vibrational level observed in the hot-band-probed dump spectrum (lower limit of $D_{0}$ ) and the lowest-frequency vibration that appears in the origin- 
probed but not in the hot-band-probed dump spectrum (upper limit of $D_{0}$ ). When taking into account the frequency shift $\delta \tilde{v}$ of the $S_{0} \rightarrow S_{1}$ origin of M.S relative to that of M, the $D_{0}\left(S_{1}\right)$ is obtained from $D_{0}\left(S_{1}\right)=D_{0}\left(S_{0}\right)-\delta \tilde{v} \cdot{ }^{38-41,43}$

The SEP-R2PI method has been applied to dispersively bound M.S complexes, ${ }^{39,43}$ to complexes involving nonclassical $\mathrm{OH} \cdots \pi$ hydrogen bonds, to aromatic and aliphatic acceptors, ${ }^{41,44}$ and to classically H-bonded complexes. ${ }^{40,41}$ The dissociation energies $D_{0}\left(S_{0}\right)$ of 10 complexes have been determined with a relative accuracy of $\approx 1 \%,{ }^{38-41,43,44}$ and are given in Table 3.

\section{Quantum Chemical Challenges for Calculating Dispersive In- teractions}

In the current issue of this journal on noncovalent interactions, a number of articles provide reviews of quantum chemical methods, computations, and benchmarks. Examples of earlier reviews are found in Refs. 45 and 46. In the present chapter, we will briefly discuss and illustrate some of the methods currently in use, thereby putting emphasis on how these methods could advantageously employ explicitly-correlated wave functions (Slater-type geminals, F12 methods). Such geminal wave functions can not only be employed in coupled-cluster theory but, for example, also within the framework of density-functional theory (DFT) in conjunction with the random-phase approximation, in dispersion-corrected double-hybrid DFT, in spin-component-scaled (SCS) methods, or in symmetry-adapted perturbation theory (SAPT), as we will see below.

We will focus our discussion on noncovalent interactions between molecules in closed-shell singlet ground states. Many of the methods discussed in this chapter can be applied to open-shell systems as well, but concerning the corresponding computational challenges, we refer to the recent work by Bozkaya and co-workers ${ }^{47,48}$ on orbital-optimized perturbation theory. See also Refs. 49 and 50. Approaches to dimers in excited states (excimers) will be briefly mentioned, but explicitlycorrelated wave functions are not yet being widely employed for calculations on excited states (for 
example in the framework of explicitly-correlated linear-response coupled-cluster theory). ${ }^{51-54}$

We will be very short on semiempirical and quantum Monte Carlo methods, because these methods are being comprehensively reviewed elsewhere in this issue. Finally, we note that it is possible to distinguish between methods that are true first-principles methods - the results of which can be improved in a systematic manner - and between methods that are more empirical in nature, that is, which contain fitted parameters (e.g., in functionals of DFT, in dispersion corrections, or in form of scaling factors). Coupled-cluster methods certainly belong to the first category while for example spin-component-scaled and DFT-based methods belong to the second category. Since the computational demands of the two categories are often very different, it may be advantageous to apply a combination of methods, for example by using a more empirical method for computations of a global PES and high-level coupled-cluster computations at selected points on that surface.

\subsection{Coupled-cluster Theory for Large Systems}

Today, highly accurate computations of the bond energies of dispersion-dominated systems are possible using approaches based on coupled-cluster theory, for example the coupled-cluster approach with iterative single and double substitutions (CCSD) augmented with noniterative, perturbative triple substitutions, that is $\operatorname{CCSD}(\mathrm{T}),{ }^{55-57}$ or its variant known as quadratic configuration interaction with singles, doubles, and noniterative triples, QCISD(T). ${ }^{55,57}$ With respect to computational details of these methods, we refer to the recent short reviews presented in Refs. 58 and 59, respectively. The $\operatorname{CCSD}(\mathrm{T})$ method — or alternatively, QCISD $(\mathrm{T})$ - is known as the "gold standard" for work on weakly bound, dispersion-dominated systems such as the benzene dimer and related systems. Such systems are small enough to allow for large basis sets such as the augmented correlation-consistent polarized valence triple-zeta basis (aug-cc-pVTZ) ${ }^{60,61}$ or larger.

The CCSD(T) approach can be applied to much larger (than the benzene dimer) hydrogen-bonded and dispersion-dominated vdW complexes by means of composite and fragment-based methods. For a recent review on such methods (of which there are numerous), see Ref. 62. Fragments can be individual molecules (monomers) or parts thereof (e.g., localized orbitals), and computations 
are performed on the individual fragments, on pairs of fragments, on triples of fragments, and so forth. Examples are the incremental scheme of Stoll, ${ }^{63-66}$ the cluster-in-molecule (CIM) approach of Li, ${ }^{67-69}$ the fragment molecular orbital (FMO) method of Kitaura, ${ }^{70-73}$ and the divideexpand-consolidate (DEC) coupled-cluster method. ${ }^{74-76}$ Furthermore, fragment-based methods can be combined with embedding techniques to yield embedded many-body expansions. ${ }^{77-79}$ In 2007, Friedrich, Hanrath, and Dolg reported a fully automated implementation of the incremental scheme ${ }^{80}$ which has since then been developed further to include explicitly-correlated coupledcluster computations. ${ }^{81-83}$

In 1983, the "localizability of dynamic electron correlation" was recognized by Pulay and exploited by means of using localized occupied Hartree-Fock orbitals in conjunction with a local virtual space that consists of atomic orbitals projected against the occupied space and each other. ${ }^{84}$ This has led to the development of local coupled-cluster (LCC) methods, ${ }^{85-89}$ which can be applied to very large systems such as vdW complexes with large chromophores. LCC methods rely on pair and domain approximations, and in particular the pair approximation has recently received attention with respect to its application to long-range intermolecular interactions. ${ }^{90-92}$ The pair approximation depends on the distance between localized occupied orbitals, and for weak intermolecular interactions, it is crucial to compute the more distant pairs (also) accurately. ${ }^{90-92}$ LCC methods have been improved by introducing pair (or pseudo) natural orbitals (PNO) in conjunction with domain approximations. ${ }^{93-98}$ Some recent applications to large systems, including the S66 test set, ${ }^{99,100}$ can be found in Refs. 101, 102, and 103. Also various explicitly-correlated variants of (PNO-based) local correlation methods have been implemented. ${ }^{101,104-108}$ In view of the progress made in recent years, one is tempted to conclude that basically from today on, explicitlycorrelated - that is, near complete-basis-set limit - CCSD(T) calculations can be performed with computational effort (computation time and other computer resources) that scales linearly with the size of the molecular system. This would render more approximate and much less accurate approaches redundant, except perhaps for optimizing the required equilibrium structures as long as analytical nuclear gradients have not yet been implemented for the (explicitly-correlated) 
PNO-based LCC methods.

\subsection{Explicitly-correlated Coupled-cluster Theory}

Explicitly-correlated coupled-cluster methods can not only be applied in a standard manner (i.e, with canonical Hartree-Fock orbitals) to rather small complexes such as the Li.benzene complex, ${ }^{109}$ but also to systems with ca. 20 atoms (with PNO-based local or incremental methods, the systems can of course be much larger). For example, in very recent work focusing on $\mathrm{N}-\mathrm{H} \cdots \pi$ interactions, CCSD(T)/aug-cc-pVTZ computations were performed for the imidazole benzene (ImBz) and pyrrole·benzene (PyBz) complexes, see Figure 9. ${ }^{110}$

In this work, the CCSD computations were performed in the large aug-cc-pVQZ basis (augmented correlation-consistent polarized valence quadruple-zeta) ${ }^{60,61}$ in conjunction with explicitly-correlated double excitations in the "(F12)" approximation ${ }^{111}$ (see Refs. 112, 113 and 114 for recent, comprehensive reviews of explicitly-correlated F12 wave functions with Slater-type geminals). This level of theory is known as the $\operatorname{CCSD}(\mathrm{F} 12) / a u g-c c-p V Q Z$ approach, and corrections for noniterative, perturbative triple substitions were computed in the aug-cc-pVTZ basis. Following Ref. 115, the latter were corrected for basis-set incompleteness by scaling the triples energy $E_{(\mathrm{T})}$ by the factor $x=E_{\mathrm{MP} 2-\mathrm{F} 12}^{\mathrm{corr}} / E_{\mathrm{MP} 2}^{\mathrm{corr}}$, where $E_{\mathrm{MP} 2-\mathrm{F} 12}^{\mathrm{corr}}$ is the explicitly-correlated second-order Møller-Plesset correlation energy and $E_{\mathrm{MP} 2}^{\mathrm{corr}}$ its conventional counterpart. Hence, the overall level of theory applied in Ref. 110 was

$$
E_{\mathrm{CCSD}(\mathrm{F} 12)+\left(\mathrm{T}^{*}\right)}=E_{\mathrm{CCSD}(\mathrm{F} 12) / \text { aug-cc-pVQZ }}+E_{\left(\mathrm{T}^{*}\right) / \text { aug-cc-pVTZ}}
$$

with

$$
E_{\left(\mathrm{T}^{*}\right) / \text { aug-cc-pVTZ }}=x E_{(\mathrm{T}) / \text { aug-cc-pVTZ }} ; \quad x=E_{\mathrm{MP} 2-\mathrm{F} 12 / \text { aug-cc-pVQZ }}^{\mathrm{corr}} / E_{\mathrm{MP} 2 / \text { aug-cc-pVTZ }}^{\mathrm{corr}} \cdot
$$

At this level of theory, the intermolecular electronic binding energies were determined to be $D_{e}=$ 22.7 and $20.4 \mathrm{~kJ} / \mathrm{mol}$ for imidazole·benzene and pyrrole·benzene, respectively, with an estimated 
uncertainty of at most a few tenths of a kJ/mol (sometimes denoted as "subchemical accuracy"). Concerning equilibrium structures, the intermolecular distances $R$ of the two complexes were optimized at the level given in Eq. (1). Computations of a scan in terms of one single intermolecular coordinate are possible today without too much computational effort, even including counterpoise corrections, ${ }^{116,117}$ but it is less straightforward to compute a counterpoise-corrected intermolecular PES at this high level of theory. Furthermore, large parts of the low-lying PES of a given dimer are required to relate the computed $D_{e}$ value to the experimentally determined dissociation energy $D_{0}$.

\subsection{Multilevel Schemes}

In attempts to alleviate the restrictions due to the very high computational costs of CCSD(T) computations on large systems, it has become common practice for computational studies of weak intermolecular interactions to approach the ab initio limit of a complete basis set (CBS) using a multilevel scheme. For example, the CBS limit of the CCSD(T) level may be approached by adding a second-order correction for the basis-set incompleteness error,

$$
\begin{gathered}
E_{\mathrm{CCSD}(\mathrm{T}) / \mathrm{CBS}} \approx E_{\mathrm{CCSD}(\mathrm{T}) / \mathrm{small} \text { basis }}+\Delta E_{\mathrm{MP} 2 / \mathrm{CBS}}, \\
\Delta E_{\mathrm{MP} 2 / \mathrm{CBS}}=E_{\mathrm{MP} 2 / \mathrm{CBS}}-E_{\mathrm{MP} 2 / \text { small basis }} .
\end{gathered}
$$

Note that, fully equivalently, Eq. (3) can also be written as

$$
\begin{gathered}
E_{\mathrm{CCSD}(\mathrm{T}) / \mathrm{CBS}} \approx E_{\mathrm{MP} 2 / \mathrm{CBS}}+\Delta E_{\mathrm{CCSD}(\mathrm{T}) / \text { small basis }} \\
\Delta E_{\mathrm{CCSD}(\mathrm{T}) / \mathrm{small} \text { basis }}=E_{\mathrm{CCSD}(\mathrm{T}) / \mathrm{small} \text { basis }}-E_{\mathrm{MP} 2 / \text { small basis }}
\end{gathered}
$$

Ref. 118 provides an example of an application of the multilevel scheme, in which the MP2 CBS limit (obtained from explicitly-correlated R12 theory), the CCSD correlation energy, and the (T) triples correction were computed in different basis sets. Recently, Pitoňák and co-workers ${ }^{119}$ have proposed a variant of Eqs. (4) and (5), denoted MP2.5, in which third-order Møller-Plesset 
perturbation theory is applied in place of $\operatorname{CCSD}(\mathrm{T})$ theory, and in which the higher-order $\Delta E$ contribution is scaled with a factor $1 / 2$,

$$
E_{\mathrm{MP} 2.5 / \mathrm{CBS}} \approx E_{\mathrm{MP} 2 / \mathrm{CBS}}+\frac{1}{2}\left(E_{\mathrm{MP} 3 / \text { small basis }}-E_{\mathrm{MP} 2 / \text { small basis }}\right)
$$

This MP2.5 approach has proven to be quite successful, taking advantage of the error cancelation between the MP2 (overestimation) and MP3 (underestimation) binding energies of dispersiondominated weakly interacting systems. Alternatively, the overestimation of MP2 theory and thus of the MP2/CBS limit can be corrected by means of an interference correction, which consists of applying individual scaling factors $0 \leq c_{i j} \leq 1$ to the pair energies $e^{(i j)}$ of the localized, occupied orbitals $i$ and $j$,

$$
\begin{gathered}
E_{\mathrm{CCSD}(\mathrm{T}) / \mathrm{CBS}} \approx E_{\mathrm{CCSD}(\mathrm{T}) / \mathrm{small} \text { basis }}+\Delta E_{\mathrm{MP} 2+\mathrm{INT} / \mathrm{CBS}} \\
\Delta E_{\mathrm{MP} 2+\mathrm{INT} / \mathrm{CBS}}=\frac{1}{2} \sum_{i j} c_{i j}\left[e_{\mathrm{MP} 2-\mathrm{CBS}}^{(i j)}-e_{\mathrm{MP} 2 / \text { small basis }}^{(i j)}\right] .
\end{gathered}
$$

Recently, ${ }^{120}$ this interference-corrected perturbation theory has been assessed with respect to the S22B database. ${ }^{121,122}$ It was also applied recently in Ref. 110 and in computations on noncovalent interactions of carbon dioxide with functional groups of metal-organic frameworks. ${ }^{123}$ Promising results were obtained, but a disadvantage of the interference-corrected approach is that it is not invariant with respect to rotations among the occupied orbitals (i.e., localized Hartree-Fock orbitals were used for that reason).

\subsection{MP2 Complete Basis-set Limit}

Often, the CBS limit at the MP2 level is obtained from MP2 computations in large (augmented) correlation-consistent basis sets followed by extrapolations to the basis set limit. In this respect, the most accurate procedure is to extrapolate individually the Hartree-Fock total energy on the one 
hand and the MP2 correlation energy on the other, as recommended in Refs. 124 and 125,

$$
\begin{gathered}
E_{\mathrm{HF} / X} \approx E_{\mathrm{HF} / \mathrm{CBS}}+A \exp (-a X), \\
E_{\mathrm{MP} 2 / X}^{\mathrm{corr}} \approx E_{\mathrm{MP} 2 / \mathrm{CBS}}^{\mathrm{corr}}+B X^{-3} .
\end{gathered}
$$

Here, $X$ is the cardinal number of the (augmented) correlation-consistent basis set. Alternatively, the MP2/CBS limit may be approached by applying the explicitly-correlated MP2 method in a (large) aug-cc-pVXZ basis,

$$
E_{\mathrm{MP} 2 / \mathrm{CBS}} \approx E_{\mathrm{HF} / X}+E_{\mathrm{MP} 2-\mathrm{F} 12 / X}^{\mathrm{corr}}+E_{\mathrm{CABS} \text { singles }}
$$

Here, $E_{\mathrm{MP} 2-\mathrm{F} 12 / X}^{\mathrm{corr}}$ approximates the CBS limit of the MP2 correlation energy while the term $E_{\mathrm{CABS}}$ singles corrects for the basis set incompleteness error in the Hartree-Fock total energy. This correction is computed from single excitations into the complementary auxiliary basis set (CABS) that is used in the explicitly-correlated methods for the resolution-of-the-identity approximation in the evaluation of many-electron integrals. An example of the use of Eq. (12) is provided by the seminal work of Sinnokrot et al. ${ }^{126}$ on the benzene dimer (in which, however, the energy contribution $E_{\mathrm{CABS} \text { singles }}$ was not needed and hence not computed, because a very large, optimized basis set of Hartree-Fock-limit quality was used for the MP2-F12 calculations). Furthermore, when using different basis sets for the MP2-F12 calculations, a Schwenke-type extrapolation can be applied to the F12 values obtained in a series of basis sets. ${ }^{127}$

By means of using Eq. (3), large weakly interacting complexes can be treated. In Ref. 128, for example, an octadecane dimer, a stacked guanine trimer, a stacked circumcoronene-adenine dimer, a stacked circumcoronene-GC dimer (GC = hydrogen-bonded guanine-cytosine Watson-Crick pair), a parallel-displaced stacked coronene dimer, two GC pairs arranged as in DNA, and a trimer of phenylalanine were studied at this level.

Besides extensively long computation times and large storage requirements, an additional problem occurs when trying to approach the MP2 CBS limit using aug-cc-pVXZ basis sets: numerical in- 
stabilities occur caused by (almost) linear dependent basis functions. In Ref. 128, this problem was avoided by using plain cc-pVXZ basis set for the extrapolation in conjunction with an empirical scaling factor to account for the difference between the aug-cc-pVXZ and cc-pVXZ sets. Also the "seasonal plantings" of diffuse functions as proposed by Papajak and co-workers ${ }^{129}$ may help to circumvent the numerical instabilities.

\subsection{Spin-component-scaled Methods}

Grimme's spin-component-scaled electron-correlation methods, ${ }^{130}$ for example SCS-MP2, are widely used in computational studies of dispersion-dominated weakly interacting complexes. These are based on the insight that low-order wave function expansions treat the correlation effects of electron pairs with opposite spin (OS) and same spin (SS) differently. In these methods, the pair energies of orbital pairs with OS and SS are scaled with individual factors $c_{\mathrm{OS}}$ and $c_{\mathrm{SS}}$, respectively,

$$
\begin{gathered}
E_{\mathrm{SCS}}^{\mathrm{corr}}=c_{\mathrm{OS}} E_{\mathrm{OS}}^{\mathrm{corr}}+c_{\mathrm{SS}} E_{\mathrm{SS}}^{\mathrm{corr}} \\
E_{\mathrm{OS}}^{\mathrm{corr}}=\sum_{i \bar{j}} e^{(i \bar{j})} ; \quad E_{\mathrm{SS}}^{\mathrm{corr}}=\frac{1}{2} \sum_{i j} e^{(i j)}+\frac{1}{2} \sum_{\bar{i} \bar{j}} e^{(\bar{i} \bar{j})} .
\end{gathered}
$$

Here, $i$ refers to an $\alpha$ spin orbital, and $\bar{i}$ to a $\beta$ spin orbital. Table 1 of Ref. 130 shows a dozen different spin-component-scaled electron-correlation methods, for example the scaled-opposite-spin method (SOS-MP2) and methods optimized for noncovalent interactions (SCS-MI-MP2, SCSNMP2, SCS-MP2-vdW). It is straightforward to optimize the coefficients $c_{\mathrm{OS}}$ and $c_{\mathrm{SS}}$ for a particular system under study.

The authors of the present review article have used the spin-component-scaled methods (mostly the original SCS-MP2 method for ground states and the SCS-CC2 method for excited states) extensively in the past, in particular for geometry optimizations. ${ }^{110,118,131,132}$ In Ref. 133, the importance of the spin-component-scaled second-order perturbation theory was emphasized for $\pi$ stacked aromatic systems while the MP2 method itself was providing excellent results for strong hydrogen bonds. An important advantage of the spin-component-scaled electron-correlation meth- 
ods is that these methods can easily and successfully be used for the treatment of low-lying valence excited states of large, weakly interacting chromophores. ${ }^{134}$ Examples are methods such as SCSCC2, SCS-ADC(2), or SCS-CIS(D). ${ }^{135-138}$

\subsection{Symmetry-adapted Perturbation Theory (SAPT)}

The starting point for SAPT is the (antisymmetrized) product of the wave functions (or Kohn-Sham determinants) ${ }^{139-141}$ of the weakly interacting monomers of the complex of interest. Then, the parts of the Hamiltonian that describe the interaction are treated as perturbation. There are various variants of SAPT that provide both the theoretical framework for analyzing intermolecular interactions and a practical tool for calculating these. ${ }^{142-145}$ For example, Sherrill has recently shown that the energy-component analysis via SAPT is very useful in understanding noncovalent $\pi$-stacking interactions and their substituent and heteroatom effects. ${ }^{146}$ Ahnen and co-workers ${ }^{110}$ have recently applied the DFT-SAPT ${ }^{147}$ approach as implemented by Heßelmann and co-workers, ${ }^{148,149}$ using the PBE0 hybrid functional ${ }^{150}$ including an asymptotic correction (PBE0AC) ${ }^{151}$ to the $\operatorname{ImBz}$ and PyBz complexes. See Table 4.

Also SAPT computations suffer from the very slow basis-set convergence that occurs when expanding dynamic-correlation functions in an orbital-product basis. One possible remedy would be to employ explicitly-correlated F12-type functions for the corresponding energy contributions, but in Ref. 110 we have simply scaled the relevant dispersion energies, $E_{\text {disp }}^{(2)}$ and $E_{\text {exch-disp }}^{(2)}$, by the ratio of the corresponding $\operatorname{CCSD}(\mathrm{F} 12)+\left(\mathrm{T}^{*}\right)$ and $\operatorname{CCSD}(\mathrm{T})$ electron-correlation contributions (in the same basis) to the interaction energy. Alternatively, a two-point $X^{-3}$ extrapolation has been applied to the aug-cc-pVDZ and aug-cc-pVTZ energies. For ImBz and PyBz, the scaled PBE0ACSAPT/aug-cc-pVTZ interaction energies were only $0.2-0.3 \mathrm{~kJ} / \mathrm{mol}$ larger in magnitude than the best estimates. 


\subsection{Dispersion-corrected Density-functional Theory}

A recent account of dispersion-corrected density-functional theory ${ }^{152}$ for aromatic interactions in complex systems is provided by Ref. 153. The most recent version of Grimme's dispersion correction is denoted "D3", which is used in conjunction with Becke-Johnson damping: D3(BJ). As an example, Table 5 shows results for the ImBz and PyBz complexes, based on GGA, hybrid, and double-hybrid functionals (GGA = generalized gradient approximation). Concerning the latter, Table 5 also shows results obtained by replacing the MP2-type correlation contribution to the double-hybrid functional by the corresponding MP2-F12 one, denoted as B2PLYP-F12, with and without frozen-core $(1 s)$ orbitals. ${ }^{110}$ Using a frozen core is unconventional but yields excellent results and a much reduced basis-set superposition errors (BSSE).

The performance of the DFT-D3(BJ) approach is impressive. The TPSSh-D3(BJ) counterpoisecorrected interaction energies of $\mathrm{ImBz}$ and $\mathrm{PyBz}$, for example, agree with the best estimates to within $0.2 \mathrm{~kJ} / \mathrm{mol} .{ }^{110}$ Table 5 also reveals that the BSSE is quite large in the aug-cc-pVTZ basis at the double-hybrid level, due to the MP2-type correlation contribution. This large BSSE is significantly reduced by computing an MP2-F12-type correlation contribution, but overall, the choice of exchange-correlation functional in DFT-D3 appears to be of little relevance for noncovalent interactions when the functional is properly dispersion corrected. Therefore, in view of computational efficiency, it seems most promising to use GGA functionals.

To conclude this subsection, we note that similarly accurate results can be obtained using the density-dependent interatomic vdW potential of Tkatchenko and Scheffler ${ }^{154,155}$ or the dispersion correction of Becke and Johnson based on the position-dependent dipole moment of the exchange hole. $^{156-158}$

\subsection{Random-phase Approximation (RPA)}

This approximation offers a computationally efficient route to the parameter-free computation of dispersion-dominated intermolecular interactions in the framework of DFT. For example, Toulouse and co-workers have developed a range-separated DFT with RPA especially for applications to 
noncovalent intermolecular interactions. ${ }^{159,160}$ For an excellent recent review on the subject, see Ref. 161. However, as it is the case for DFT-based SAPT (vide supra), expanding short-range dynamic-correlation functions in an orbital-product basis leads to slow basis-set convergence. ${ }^{162}$ Moreover, methods that go beyond the "direct" random-phase approximation (i.e., methods that go beyond using the Hartree kernel only) exhibit an unphysical short-range behavior of the correlated pair function in large basis sets. ${ }^{163}$ Recent developments include a self-consistent treatment of electron correlation in "direct" RPA (dRPA), ${ }^{164}$ the analytical calculation of nuclear gradients, ${ }^{165}$ the implementation of quasi-relativistic two-component (spin-orbit including) RPA methods, ${ }^{166,167}$ and a linear-scaling implementation. ${ }^{168}$

Addressing the basis-set problems, an explicitly-correlated F12-type correction to the dRPA correlation energy has recently been implemented. ${ }^{169}$ The corresponding method is denoted dRPA(functional) $+\mathrm{F} 12$, where "functional" is the exchange-correlation functional used in the underlying Kohn-Sham calculation. Also a dRPA(functional)(F12) method has been implemented, which is an explicitly-correlated direct ring-coupled-cluster-doubles method, drCCD(F12), with KohnSham orbitals. ${ }^{170}$ Table 6 shows corresponding dRPA(PBE)+F12 and -(F12) results for ImBz and PyBz.

We expect that F12 corrections will also dramatically improve the basis-set convergence of the correlation energy in the random-phase approximation when exchange terms are taken into account, as for example in calculations with the approximate exchange kernel (AXK) ${ }^{171}$ or in the second-order screened exchange (SOSEX) approximation. ${ }^{172,173}$

We conclude this subsection by pointing out that the range-separated RPA method of Toulouse and co-workers ${ }^{159,160}$ does not suffer from a slow basis set convergence, because in this method only a long-range RPA correlation energy is computed. ${ }^{174}$ 


\subsection{Quantum Monte Carlo and Semiempirical Methods}

Finally, also semiempirical methods may be applied to dispersion-dominated weakly interacting complexes when empirical corrections for dispersion (and possibly also hydrogen bonding) are added. Examples are the methods PM6, AM1 and OM3 ${ }^{175}$ as well as the SCC-DFTB-D method. ${ }^{175-177}$ These methods were shown to reach the quality of current DFT-D approaches for noncovalent interactions. ${ }^{175}$

Furthermore, it has recently been shown that the fixed-nodes diffusion Monte Carlo (FN-DMC) ap-

proach may be seen as an interesting alternative to the "gold standard" CCSD(T)/CBS method. ${ }^{178-180}$

\subsection{Excited States $\left(S_{1}\right)$ of Weakly Interacting Complexes}

While there are numerous methods available for the computation of the binding energy of a weakly interacting complex in its ground state $\left(S_{0}\right)$, this is no longer true for weakly interacting complexes in their first singlet excited state $\left(S_{1}\right)$. Appropriate methods are the (SCS-)CC2 linear-response coupled-cluster method or multireference approaches such as the CASSCF/CASPT2 approach (complete-active-space self-consistent field plus second-order perturbation theory). Recent examples are found in Refs. 181 and 182 respectively. Also time-dependent density-functional theory (TD-DFT) may be applicable when using appropriate functionals, as shown for example by Huenerbein and Grimme for organic excimers of pyrene and benzene and an exciplex of styrene with trimethylamine. ${ }^{183}$

In our own work, we have used the linear-response SCS-CC2 method as implemented in the program system TURBOMOLE. ${ }^{184,185}$ Due to its efficient implementation using the resolution-ofthe-identity approximation, it is today possible to compute the harmonic vibrational frequencies of a trimer consisting of imidazole and two benzene molecules at the SCS-CC2/def2-QZVPP 186 level, see Figure 10.

After optimizing the geometry of the (benzene) $)_{2} \cdot$ imidazole trimer in its ground state at the SCSMP2/def2-QZVPP level and its first singlet excited state at the SCS-CC2/def2-QZVPP level, the 0-0 transition is found at $4.72 \mathrm{eV}$ when including the (harmonic) zero-point vibrational energy 
(ZPVE). It is assigned to the $1{ }^{1} B_{2 u} \leftarrow 1{ }^{1} A_{1 g} 0-0$ transition of benzene. ${ }^{187}$ The energy difference at the individual equilibrium structures ( $T_{e}$ value) amounts to $4.91 \mathrm{eV}$. In the present review article, however, we are not concerned with the computations of binding energies of weakly interacting complexes in excited states.

\subsection{Zero-point Vibrational Energy}

Possible methods to compute the anharmonic zero-point vibrational energy (ZPVE) of weakly interacting complexes include quantum diffusion Monte Carlo (as recently applied to the $\mathrm{H}_{2} \cdot \mathrm{Li}^{+}$.benzene system) ${ }^{188}$ or methods that diagonalize the nuclear Hamiltonian in a discrete-variablerepresentation (DVR) basis, as pioneered by Bačić and co-workers in the 1990s (using summed empirical atom-atom Lennard-Jones potentials) ${ }^{189-193}$ and as for example recently done for PESs of aromatics with rare gases $\mathrm{R}$ in ground and excited states. ${ }^{194-196}$

Such methods need a full-dimensional intermolecular PES, which for example can be computed using the modified Shepard interpolation of Bettens and co-workers. ${ }^{197}$ Note, however, that these PESs represent the calculated fixed-fragments interaction energy $\Delta E\left(R_{\min , \text { inter }}\right)$, which neglects the geometrical relaxation of $\mathrm{M}$ and $\mathrm{S}$. Thus the intermolecular electronic binding energy $D_{e}$ is not the minimum of this PES, but needs to be calculated separately. For type-II and type-III complexes with an aromatic $\mathrm{M}$ and a rare gas $\mathrm{R}$, the (reduced) masses are relatively large, and the total ZPVE of the three intermolecular vibrations is in the range of $30-50 \mathrm{~cm}^{-1}$. We estimate the error of the harmonic treatment to be $5-10 \mathrm{~cm}^{-1}$; this is small relative to current error in calculated $D_{e}$. Hobza and coworkers have recently shown that $\triangle \mathrm{ZPVE}$ corrections terms for dispersion-bound complexes are small, both harmonic and anharmonic $D_{0}$ values being in good agreement with experimental results. ${ }^{10}$

Alternatively, vibrational second-order perturbation theory (VPT2) may be applied, as for example done for the (hydrogen-bonded) formic acid dimer in Ref. 198 using anharmonicity constants computed at the DFT level. Vibrational configuration interaction (VCI) can also be applied to molecular complexes, as demonstrated recently by Panek and Jacob for the intramolecular vibra- 
tions of the water tetramer using localized modes. ${ }^{199}$ It can of course be used in conjunction with the explicitly-correlated coupled-cluster approach. ${ }^{200}$

\section{Examples of Binding and Dissociation Energies of Disper- sively Bound Complexes}

\subsection{Type I Complexes: Two Systems with Localized HOMOs.}

\subsection{1 $\mathrm{Ar}_{2}, \mathrm{Kr}_{2}, \mathrm{Xe}_{2},\left(\mathrm{~N}_{2}\right)_{2}$ and $(\mathrm{CO})_{2}$}

Due to their simplicity and significance for understanding non-covalent interactions, noble-gas homo- and heterodimers and the related dimers $\left(\mathrm{N}_{2}\right)_{2}$ and $(\mathrm{CO})_{2}$ are paradigmatic examples of type I complexes. The ionization properties and ion ground-state $D_{0}$ values of $\mathrm{Ar}_{2},\left(\mathrm{~N}_{2}\right)_{2}$ and $(\mathrm{CO})_{2}$ have been reviewed by Weitzel and Mähnert. ${ }^{201}$

The ground-state ${ }^{1} \Sigma_{g}^{+}$potential curve of $\mathrm{Ar}_{2}$ has been extracted from the VUV absorption spectrum, ${ }^{202}$ yielding $D_{e}=1.18 \mathrm{~kJ} / \mathrm{mol}, D_{0}=1.03 \mathrm{~kJ} / \mathrm{mol}$ and a minimum-energy distance $R_{e}\left({ }^{1} \Sigma_{g}^{+}\right)=$ $3.761 \AA .{ }^{203}$ Measuring the $\mathrm{Ar}_{2}^{+2} \Sigma_{u}^{+}$ground state and the AIE of $\mathrm{Ar}_{2}$ has proven challenging: Due to charge-resonance stabilization in the ${ }^{2} \Sigma_{u}^{+}$state, the equilibrium distance shortens to $R_{e}\left({ }^{2} \Sigma_{u}^{+}\right)$ $=2.32 \AA,{ }^{204}$ and the Franck-Condon overlap between the ${ }^{1} \Sigma_{g}^{+}$and ${ }^{2} \Sigma_{u}^{+}$state $v=0$ wave functions is essentially zero, see Figure 3. Despite this, two research groups have been able to probe $\mathrm{Ar}_{2}^{+}$ground state vibrational levels from $v^{+}=3-52$ using pulsed-field ionization zero electronic kinetic energy (PFI-ZEKE) spectroscopy. ${ }^{204-206}$ The Franck-Condon factors to vibrations with $v^{+}<25$ are negligible, ${ }^{204}$ and the appearance of these bands has been explained by "channel interactions" with high-lying Rydberg series of $\mathrm{Ar}_{2}$ that converge to electronically excited states of $\mathrm{Ar}_{2}^{+}$. Although the detailed mechanism is not clear, the presence of these low- $v$ bands in the PFI-ZEKE spectrum allows to fit the potential energy curve, yielding AIEs of $14.455 \mathrm{eV}^{205}$ or $14.457 \mathrm{eV}^{206}$ (the differences stem from the different fitting approaches). ${ }^{206}$ The resulting dissociation energy of $\mathrm{Ar}_{2}^{+}$is $D_{0}=127 \mathrm{~kJ} / \mathrm{mol}$, which is $>100 \mathrm{x}$ larger than that of the $\mathrm{Ar}_{2}{ }^{1} \Sigma_{g}^{+}$ground 
state. As expected from the poor Franck-Condon factors around the spectroscopic origin, ionization energies determined just by the appearance of photoions or photoelectrons tend to overestimate the AIE.

Similar observations have been made for $\mathrm{Kr}_{2}$ and $\mathrm{Xe}_{2}:{ }^{207,208}$ While the ionizative Franck-Condon factors are expected to give spectroscopic access only to vibrational states $\mathrm{v}^{+} \approx 30-60$, channel interactions with high Rydberg states allowed to identify lower vibrational levels when using the PFI-ZEKE technique. In these cases, even the ion ground state $v^{+}=0$ level was identified, allowing a very accurate description of the cation potential curve. Results of comparable quality have also been obtained for the KrXe and ArXe noble-gas heterodimers. ${ }^{209,210}$ This renders PFI-ZEKE a valuable tool for the determination of the AIEs of type I complexes, utilizing thermochemical cycles for the neutral ground state dissociation energies.

\subsection{2 $\left(\mathrm{H}_{2} \mathrm{O}\right)_{2},\left(\mathrm{D}_{2} \mathrm{O}\right)_{2}, \mathrm{H}_{2} \mathrm{O} \cdot \mathrm{HCl}$ and $\left(\mathrm{NH}_{3}\right)_{2}$}

While the water dimer is H-bonded and is outside the topic of this review, it represents a further type I complex. Furthermore, Reisler and co-workers have recently performed elegant velocity map imaging (VMI) measurements of the dissociation energies of $\left(\mathrm{H}_{2} \mathrm{O}\right)_{2}$ and $\left(\mathrm{D}_{2} \mathrm{O}\right)_{2}$ that beautifully illustrate the power of the VMI technique. ${ }^{211,212}$

The water dimers are dissociated by pumping the $\mathrm{O}-\mathrm{H}$ stretch vibration in the ground state using an IR laser pulse, followed by IVR and vibrational predissociation. The two $\mathrm{H}_{2} \mathrm{O}$ fragments are then ionized using a 2+1 REMPI scheme (resonance enhanced multiphoton ionization) and their velocity distribution is measured by VMI. Since the rotational levels of $\mathrm{H}_{2} \mathrm{O}$ are well-separated, and because the excess energy of the IR photon above $D_{0}$ suffices only to populate $v=1$ of the bending vibration of one of the $\mathrm{H}_{2} \mathrm{O}$ fragments, the rotational and vibrational energies of the ionized fragments are determined state-specifically and their respective translational energy distribution yields detailed information on the dissociation process. Apart from $D_{0}$, the other unknown parameters are the rotational and vibrational energy of the co-fragments. However, these define the shape of the velocity distributions, and could be established by fitting the velocity distribu- 
tion curves obtained by monitoring the velocity distribution of the $\mathrm{H}_{2} \mathrm{O}$ fragments in four different rovibrational states. ${ }^{211}$ The dissociation energies of $D_{0}=13.2 \pm 0.11 \mathrm{~kJ} / \mathrm{mol}$ for $\left(\mathrm{H}_{2} \mathrm{O}\right)_{2}$ and $D_{0}=14.9 \pm 0.12 \mathrm{~kJ} / \mathrm{mol}$ for $\left(\mathrm{D}_{2} \mathrm{O}\right)_{2}$ are in excellent agreement with theory. ${ }^{213,214}$ Remarkably, the calculated binding energy $D_{e}$ amounts to $20.8-21.0 \mathrm{~kJ} / \mathrm{mol},{ }^{215}$ being ca. $60 \%$ larger than $D_{0}$ ! The water dimer is an impressive example of the importance of ZPVE when comparing theory to experiment. Note also that the dissociation energy changes by $\approx 13 \%$ upon deuteration.

In contrast to other examples of VMI discussed in Section 3.4, the ground state $D_{0}$ is here determined directly from the dissociation kinematics plus detailed spectroscopic knowledge of the monomer and dimer rotational and vibrational energies, without employing thermochemical cycles. The technique has therefore only been applied to small hydride dimers and complexes with well-separated rotational vibrational states. Other complexes studied by VMI so far are $\mathrm{H}_{2} \mathrm{O} \cdot \mathrm{HCl}$ $\left(D_{0}=15.95 \pm 0.12 \mathrm{~kJ} / \mathrm{mol}\right)^{216}$ and $\left(\mathrm{NH}_{3}\right)_{2}\left(D_{0}=7.89 \mathrm{~kJ} / \mathrm{mol}\right) .{ }^{217}$

An analogous approach has been applied earlier by the group of Roger Miller, who determined the dissociation energies of a large number of small complexes using photofragment angular distribution (PHOFAD) or position-sensitive translational spectroscopy (POSTS) methods. ${ }^{218}$ In this approach, the kinetic energy distribution of the photofragment moieties was probed by recording their deflection angle from the molecular beam axis using a movable liquid He-cooled bolometer. The results of these studies are compiled in Table 1. Due to the quality of the data, it is recommended that this approach should be exploited whenever it is feasible.

\subsection{Type II Complexes: A System with Delocalized HOMO and a System with a Localized HOMO}

\subsubsection{Benzene·Argon}

Measurements of the ground-state dissociation energy of benzene-argon (Bz-Ar) have been performed by Neusser and co-workers using ion breakdown measurements $\left(D_{0}=1.6 \pm 0.5 \mathrm{~kJ} / \mathrm{mol}\right)^{32}$ and later by pulsed field mass analyzed threshold ionization (PFI-MATI) spectroscopy $\left(D_{0}<4.1\right.$ 
$\mathrm{kJ} / \mathrm{mol})^{27,219}$. More recently, Lawrance and co-workers ${ }^{34}$ have provided a more accurate $D_{0}=$ $3.77 \pm 0.08 \mathrm{~kJ} / \mathrm{mol}$ using velocity map imaging (VMI). While the lower limit of the PFI-MATI measurement agrees with the VMI value, the breakdown measurement ${ }^{32}$ yields a much smaller dissociation energy (cf. Table 2).

In the VMI experiment, the dissociation energy has been determined from the cycle $D_{0}(\mathrm{Bz} \cdot \mathrm{Ar})$ $=\mathrm{D}_{0}^{+}\left(\mathrm{Bz}^{+} \cdot \mathrm{Ar}\right)+\mathrm{AIE}(\mathrm{Bz})$. The AIEs of $\mathrm{Bz} \cdot \mathrm{Ar}$ and $\mathrm{Bz}$ were previously determined using PFIZEKE spectroscopy. ${ }^{220}$ The cation dissociation energy $D_{0}^{+}$was determined by ionizing $\mathrm{Bz} \cdot \mathrm{Ar}$ into a vibrational state above the $D_{0}^{+}\left(\mathrm{Bz}^{+} \cdot \mathrm{Ar}\right)$ dissociation limit. Thereby, the excess energy was chosen as small as possible to prevent the formation of vibrationally excited fragments. As an intermediate state for the two-photon ionization, the $6^{1}$ level of the $S_{1}$ state, $521 \mathrm{~cm}^{-1}$ above the electronic origin was chosen. VMI was then used to measure the translational energy distribution of the $\mathrm{Bz}^{+}$fragments. Subtracting the $\mathrm{AIE}(\mathrm{Bz} \cdot \mathrm{Ar})$ and the highest observed kinetic energy of the fragment from the excitation energy yielded $D_{0}^{+}=5.81 \pm 0.05 \mathrm{~kJ} / \mathrm{mol}$. This approach assumes that the $\mathrm{Bz}^{+}$is formed in the $N^{+}=0$ rotational level during VP. Thus, the $D_{0}^{+}$value evaluated by VMI is an upper limit.

The intermolecular potential of Bz·Ar has been constructed based on CCSD(T)/aug-cc-pVDZ calculations, resulting in a fixed-fragments interaction energy $\Delta E\left(R_{\min , \text { inter }}\right)=-4.66 \mathrm{~kJ} / \mathrm{mol} .{ }^{221}$ This value agrees with the value of $-4.65 \mathrm{~kJ} / \mathrm{mol}$ reported in Ref. 222 , which was obtained by combining the finite-basis MP2 and $\operatorname{CCSD}(\mathrm{T})$ values of -5.54 and $-3.57 \mathrm{~kJ} / \mathrm{mol}$, respectively, with the infinite-basis MP2 limit of $-6.62 \mathrm{~kJ} / \mathrm{mol}$. Assuming a negligible deformation of benzene by the interaction with the Ar atom, this value may be identified also as the binding energy $D_{e}$. Zero-point correction of the $D_{e}$ using the three intermolecular frequencies calculated from the intermolecular potential of Ref. 221 leads to $D_{0}=3.83 \mathrm{~kJ} / \mathrm{mol}$, which is in excellent agreement with the results of Lawrance and co-workers. ${ }^{34}$ 


\subsubsection{Complexes with Carbazole}

Ground-state dissociation energies $D_{0}$ of complexes of carbazole with noble gas atoms (Ne, $\mathrm{Ar}, \mathrm{Kr}$, $\mathrm{Xe})$ and small molecules $\left(\mathrm{CO}, \mathrm{N}_{2}, \mathrm{CH}_{4}\right)$ have been measured by the stimulated-emission pumping/resonant two-photon ionization (SEP-R2PI) method. ${ }^{38,39,43}$ To illustrate a comparison of experiment with calculation, we have optimized the equilibrium structures of these complexes (and of course also those of the carbazole molecule, $\mathrm{CO}, \mathrm{N}_{2}$, and $\mathrm{CH}_{4}$ ) at the SCS-CC2/aug-cc-pVTZ level, see Section 4.5. Figure 11 shows the optimized equilibrium structures of carbazole. $\mathrm{CO}$ and carbazole $\cdot \mathrm{CH}_{4}$.

At these equilibrium structures, we have performed single-point energy calculations at the (SCS)MP2-F12/cc-pVTZ-F12 $223-225$ level using the TURBOMOLE program ${ }^{184,185,226}$ and at the DFTSAPT/aug-cc-pVTZ level with the MOLPRO program. ${ }^{227,228}$ Concerning DFT-SAPT, we have added a $\Delta$ F12 correction that has been obtained by performing an MP2-F12 calculation in which only those second-order pair energies are taken into account where the two occupied orbitals are localized on different fragments (using MOLPRO's Kohn-Sham orbitals and orbital energies). We refer to this level as SAPT-F12(MP2). The PBE0AC functional has been applied using experimental values for the ionization energies. ${ }^{151}$ ZPVEs have been computed in the harmonic approximation at the PBE-D3/def2-TZVP level. ${ }^{229,230}$ The computed dissociation energies $D_{0}$ are summarized in Figure 12. Clearly, dissociation energies are strongly overestimated at the MP2F12 level while the spin-component-scaled SCS-MP2-F12 energies are much improved for these dispersion-dominated weakly interacting vdW complexes.

Table 7 compares the MP2-F12, SCS-MP2-F12, and SAPT-F12(MP2) results with the experimental $D_{0}$ values. The agreement of the SAPT-F12(MP2) results with the experimental values is quite satisfactory, the largest deviation being $-1 \mathrm{~kJ} / \mathrm{mol}$ for carbazole $\cdot \mathrm{CO}$. Note, however, that the experimental uncertainty for this complex is $\pm 0.38 \mathrm{~kJ} / \mathrm{mol}$. The individual contributions to the SAPT-F12(MP2) binding energies are shown in Table 8.

As in Ref. 38, we plot the SAPT-F12(MP2) dispersion energy $E_{\text {disp }}^{(2)}$ and the computed binding energy $D_{e}$ of the complexes of carbazole with the noble gases X against the static polarizability $\alpha_{\mathrm{X}}$ 
of these atoms in Figure 13. Clearly these energies depend linearly on the atomic polarizabilities, in accord with London's formula. The polarizabilities $\alpha_{X}$ were multiplied with the ionization energy factor $I_{X} I_{\mathrm{Carb}}\left(I_{\mathrm{X}}+I_{\mathrm{Carb}}\right)^{-1}$ with $I_{\mathrm{Carb}}=7.57 \mathrm{eV}$ and $I_{\mathrm{X}}=21.55,15.75,14.00$, and $12.13 \mathrm{eV}$ for $\mathrm{Ne}, \mathrm{Ar}, \mathrm{Kr}$, and $\mathrm{Xe}$, and the polarizabilities were divided by the sixth power of the interfragment distances $R=3.11,3.32,3.40$, and $3.43 \AA$ that were obtained at the SCS-CC2/aug-cc-pVTZ level. Note that the electronic binding energy $D_{e}$ is plotted.

Further examples of experimentally determined and computed dissociation energies of type II complexes with small molecules are compiled in Table 9.

\subsection{Type III Complexes: Two Systems with Delocalized HOMOs}

We denote dispersively bound self-dimers $\mathbf{M}_{2}$ of aromatic molecules as type III complexes (cf. Table 10). Several problems arise when trying to determine their dissociation energies, which are exemplified using the benzene dimer, $\mathrm{Bz}_{2}$.

\subsubsection{Benzene Dimer}

Multiple ground-state structures: Due to the weakness and weak directionality of dispersive intermolecular interactions, the PESs of type III complexes are relatively shallow and exhibit multiple minima, rendering notions such as that of "structure" problematic. For decades $\mathrm{Bz}_{2}$ was predicted to have a "stacked" or "slipped-stacked" minimum-energy structure with parallel benzene planes. However, already in 1975, molecular-beam electric-deflection measurements showed that isolated and supersonically cooled $\mathrm{Bz}_{2}$ is polar, and a T-shaped structure was suggested. ${ }^{231}$ In 1993 , a Tshaped structure with a distance of $5.5 \AA$ between the centers-of-mass of the "T-stem" and "T-cap" $\mathrm{Bz}$ moieties was inferred from the microwave rotational spectrum of $\mathrm{Bz}_{2} .{ }^{232}$ In 2006, Podeszwa et al. ${ }^{233}$ calculated a 6-dimensional intermolecular PES for $\mathrm{Bz}_{2}$ using DFT-based symmetry-adapted perturbation theory (SAPT) and predicted the tilted T-shaped geometry M2 shown in Figure 14 as the global minimum. The M2 minimum (with $D_{e}=11.6 \mathrm{~kJ} / \mathrm{mol}$ ) was calculated to be slightly below the slipped-stacked local minimum denoted M1 $\left(D_{e}=11.5 \mathrm{~kJ} / \mathrm{mol}\right)$ and much lower than a 
previously unknown twisted edge-to-edge local minimum denoted $\mathbf{M 3}\left(D_{e}=7.6 \mathrm{~kJ} / \mathrm{mol}\right)$.

Large-amplitude motions, structural interconversions and internal tunneling processes: The symmetrictop character of the microwave spectrum ${ }^{232}$ is surprising because any rigid $\mathrm{Bz}_{2}$ structure is an asymmetric top. Indeed, the microwave spectrum exhibits multiple splittings that reflect rapid structural interconversion processes, ${ }^{234-236}$ which occur in the vibrational ground state and interchange the $\mathrm{Bz}$ monomers between 48 degenerate (but structurally distinct) forms of M2. ${ }^{234}$ Based on an improved third-order DFT-SAPT PES and supplementary CCSD(T) calculations, van der Avoird and co-workers identified three distinct tunneling paths that interconvert energetically degenerate forms of $\mathbf{M} 2$ (hindered cap $C_{6}$ internal rotation, tilt tunneling of the stem Bz, cap turnover). ${ }^{234,235}$ Furthermore, The $\mathrm{Bz}_{2}$ DFT-SAPT PES of Podeszwa et al. ${ }^{233}$ revealed four lowlying index-1 saddle points, denoted $\mathbf{S 1}$ to $\mathbf{S 4}$, that lie merely $0.1-0.5 \mathrm{~kJ} / \mathrm{mol}$ above the minima, indicating that structural interconversions between M1 and M2 are also facile.

Large intermolecular vibrational zero-point energies: Based on the calculation of the vibration-rotation-tunneling levels of $\mathrm{Bz}_{2},{ }^{234,235}$ the vibrational zero-point energy of the six intermolecular vibrations is predicted to be ZPVE $=1.26 \mathrm{~kJ} / \mathrm{mol}$. This amounts to nearly $11 \%$ of the calculated well depth of the T-shaped M2 minimum fixed-fragment interaction energy of $\Delta E\left(R_{\text {min,inter }}\right)=11.6 \mathrm{~kJ} / \mathrm{mol} .^{234,235}$ As shown in Figure 15 , the ZPVE places the lowest $v=0$ intermolecular vibrational level of $\mathrm{Bz}_{2}$ well above the $\mathbf{S 1}$ to $\mathbf{S} 4$ saddle points of the intermolecular PES.

Large differences between the PESs of the neutral and ion ground states: As shown schematically in Figure 15 for $\mathrm{Bz}_{2}$, the neutral ground-state PES of $\mathrm{M}_{2}$ and the ion ground PES of $\mathrm{M}_{2}^{+}$can differ dramatically. The ground-state minimum-energy structure of $\mathrm{Bz}_{2}$ is T-shaped while the $\mathrm{Bz}_{2}^{+}$ minimum-energy structure is slipped-stacked (M1).

Experimental dissociation energy of $\mathrm{Bz}_{2}$ : The dissociation energy of $\mathrm{Bz}_{2}$ has been measured by Hui and co-workers ${ }^{237}$ who reported $D_{0}=10.0 \pm 1.7 \mathrm{~kJ} / \mathrm{mol}$ and by Neusser and co-workers, ${ }^{25,238}$ who reported a distinctly smaller value, $D_{0}=6.75 \pm 1 \mathrm{~kJ} / \mathrm{mol}$. Two different thermochemical 
cycles where applied: Hui exploited the relationship given in Eq. (15), cf. Figure 5.

$$
D_{0}=D_{0}^{+}+\mathrm{AIE}\left(\mathrm{Bz}_{2}\right)-\mathrm{AIE}(\mathrm{Bz})
$$

Using one-photon VUV photoionization, Hui and co-workers measured $\mathrm{AIE}\left(\mathrm{Bz}_{2}\right)=8.690 \pm 0.023$ $\mathrm{eV}{ }^{237}$ To obtain the dissociation energy of the cation, $D_{0}^{+}$, they extrapolated the value $D_{0}^{+}=$ $71.1 \pm 6.3 \mathrm{~kJ} / \mathrm{mol}$ obtained by Field et al. ${ }^{13}$ from equilibrium measurements at $300 \mathrm{~K}$ down to $0 \mathrm{~K}$ using "reasonable estimates for the vibrational modes involved", ${ }^{237}$ resulting in $D_{0}^{+}=69.8 \pm 7.0$ $\mathrm{kJ} / \mathrm{mol}$. This uncertainty results in a very broad range for $D_{0}$ between 9.3 and $23.3 \mathrm{~kJ} / \mathrm{mol}$. Upon including the results of IR spectra of Nishima and Hanazaki ${ }^{239}$ who reported an upper limit for $D_{0}\left(\mathrm{Bz}_{2}\right)$ as $11.87 \mathrm{kcal} / \mathrm{mol}$, Hui and co-workers arrived at a final result of $D_{0}=10.0 \pm 1.7 \mathrm{~kJ} / \mathrm{mol}$. In contrast, Neusser and co-workers based their $D_{0}$ determination on the measurement of the appearance energy $(\mathrm{AE})$ of the $\mathrm{Bz}^{+}$daughter ion from the ion breakdown of $\left(\mathrm{Bz}_{2}\right)^{+}$, using the thermochemical cycle $D_{0}=\mathrm{AE}\left(\mathrm{Bz}^{+}\right)-\mathrm{AIE}(\mathrm{Bz})$ (see also Figure 5). From their measured $\mathrm{AE}\left(\mathrm{Bz}^{+}\right)=9.31 \mathrm{eV}$ and $\mathrm{AIE}(\mathrm{Bz})=9.24378 \pm 0.00007 \mathrm{eV}$ as above, ${ }^{240,241}$ they obtained a $\mathrm{Bz}_{2}$ dissociation energy of $D_{0}=6.75 \pm 1 \mathrm{~kJ} / \mathrm{mol}$. When compared to the calculated $D_{0}=10.34 \mathrm{~kJ} / \mathrm{mol}$ of Avoird and coworkers, ${ }^{234,235}$ this amounts to a difference of about $4 \mathrm{~kJ} / \mathrm{mol}$ or $50 \%$.

Repeated measurements of VUV one-photon or resonant two-color two-photon ionization (2CR2PI) photoionization efficiency (PIE) curves of $\mathrm{Bz}_{2}$ by the groups of Schlag and Baumgärtel resulted in values for $\mathrm{AIE}\left(\mathrm{Bz}_{2}\right)$ ranging from $8.865 \mathrm{eV}^{242}$ to $8.65 \mathrm{eV},{ }^{242-244}$ the latter value being slightly below the Hui and co-workers value of $8.690 \mathrm{eV} .^{237}$ However, when inserting the calculated $D_{0}^{+}=86.6 \mathrm{~kJ} / \mathrm{mol}$ of Krylov ${ }^{245}$ and the calculated ZPVE-corrected fixed-fragment interaction energy $-10.34 \mathrm{~kJ} / \mathrm{mol}$ of Avoird et al. ${ }^{234,235}$ into Eq. (15), one arrives at $\mathrm{AIE}\left(\mathrm{Bz}_{2}\right)=8.453 \mathrm{eV}$, which is $0.2 \mathrm{eV}$ below the lowest experimental value. The reason for this are the large changes between the $\mathrm{Bz}_{2}$ and $\left(\mathrm{Bz}_{2}\right)^{+}$minimum geometries, which lead to minute Franck-Condon overlaps in ionization, rendering both one-photon and two-photon ionization measurements of the AIE of $\mathrm{Bz}_{2}$ nearly impossible. The large errors of the AIE values has a devastating effect on all subsequent 
thermochemical cycles.

Therefore, experimental techniques that rely on measurements involving the neutral ground state are recommended. One approach has been presented by Gentry and co-workers who measured the photodissociation dynamics of $\mathrm{Bz}_{2}$ upon excitation of the $v_{18}$ level at $1038 \mathrm{~cm}^{-1}(12.4 \mathrm{~kJ} / \mathrm{mol})$, obtaining dissociation times of 2.5 ps. ${ }^{246}$ Felker and co-workers used stimulated vibrational Raman scattering (SRS) to populate the $v_{1}$ fundamental of the $\mathrm{Bz}_{2}$ complex at $992 \mathrm{~cm}^{-1}(11.87 \mathrm{~kJ} / \mathrm{mol}) .{ }^{247}$ The Raman-excited complex was subsequently detected by one-color R2PI. Employing a time delay of $\Delta t \approx 50 \mathrm{~ns}$ between the SRS pulses and the excitation/ionization laser pulse, no decrease of the vibrationally excited population was observed. This led Felker and co-workers to the conclusion that either VP occurs on $\gg 50 \mathrm{~ns}$ time scale or that $D_{0}>11.87 \mathrm{~kJ} / \mathrm{mol}$. This yields limits between $11.87<D_{0}<12.4 \mathrm{~kJ} / \mathrm{mol}$, which is in acceptable agreement with the calculated ZPVEcorrected fixed-fragment interaction energy of $-10.4 \mathrm{~kJ} / \mathrm{mol}{ }^{234,235}$ This value is based on the calculated $\Delta E\left(R_{\text {min,inter }}\right)$ of the 6-dimensional rigid-monomer PES and the anharmonic ZPVE of the six intermolecular vibrations, given as $105 \mathrm{~cm}^{-1}=1.26 \mathrm{~kJ} / \mathrm{mol}$ in ref. 234. However, $\mathrm{Bz}_{2}$ has 66 vibrations, of which the 60 intramolecular vibrations are neglected in the rigid-monomer treatment. The effect of dimer formation on these is not known; it could increase or decrease the $\triangle \mathrm{ZPVE}$.

\subsubsection{Toluene Dimer}

Despite their similar chemical structure, the conformational space of (toluene) ${ }_{2}$ and $\mathrm{Bz}_{2}$ differ markedly, since T-shaped structures of the toluene dimer are either calculated to be substantially less favorable ${ }^{248,249}$ than $\pi$-stacked conformers, or could not even be identified as minima. ${ }^{250}$ This change of conformational preferences has been ascribed to (1) the compensation of the repulsive quadrupole-quadrupole interactions (which disfavor stacked conformations in $\mathrm{Bz}_{2}$ ) by an attractive dipole-dipole interaction in stacked (toluene) 2 , and (2) the increase in the dispersive interaction due to the additional methyl group. ${ }^{249}$ A decomposition of the interaction energy ${ }^{248}$ shows that the electrostatic contribution barely changes, while there is an increase of the correlation energy 
by $\approx 40 \%$ on going from $\mathrm{Bz}_{2}$ to (toluene) $)_{2}$.

Three different local minima of stacked (toluene) $)_{2}$ have been identified with the $\mathrm{CH}_{3}$-groups of the toluene moieties either parallel ( $C_{2 h}$ symmetry), anti-parallel $\left(C_{s}\right)$ or at a $\approx 90^{\circ}$ angle ("crossisomer", $C_{2}$ symmetric). ${ }^{248,250}$ The interaction energies of these three isomers are calculated to be very similar. The most stable isomer is predicted to be either the cross $\left(\Delta E_{2}=-17.1 \mathrm{~kJ} / \mathrm{mol}\right.$, estimated $\operatorname{CCSD}(\mathrm{T}) / \mathrm{CBS})^{248}$ or the anti-parallel isomer with $\Delta E_{2}=-14.5 \mathrm{~kJ} / \mathrm{mol}(\mathrm{CCSD}(\mathrm{T}) / 6$ $\left.311++\mathrm{G}^{* *}\right) .{ }^{250}$ We note a considerable difference between the interaction energies evaluated with the two applied protocols.

The $0_{0}^{0}$ band of the $S_{0} \rightarrow S_{1}$ spectrum of (toluene) $)_{2}$ is only $\approx 100 \mathrm{~cm}^{-1}$ wide and consists of sharp transitions and an underlying broad absorption. ${ }^{251}$ Hole-burning spectroscopy revealed the presence of at least two different isomers, ${ }^{252}$ which were first assigned to a stacked and a T-shaped structure. ${ }^{251}$ More recent calculations ${ }^{248,250}$ suggest that two (or more) $\pi$-stacked isomers coexist in the molecular beam, but their identification is hindered by the absence of distinct spectroscopic features. The dissociation energy has been determined via breakdown measurement $(\mathrm{BM})$ as $D_{0}=$ $14.5 \pm 1 \mathrm{~kJ} / \mathrm{mol} .^{25}$ It remains unclear which of the isomers was been probed in that investigation. On the other hand, the experimental uncertainty of the $D_{0}$ is larger than the calculated energy differences between the various isomers, so even if the method was able to probe the different isomers, it could not resolve the differences of the dissociation energies.

\subsubsection{Indole-Benzene}

The indole benzene dimer provides a gas-phase model for the interactions between aromatic amino acids on protein structures, mimicking the tryptophan phenylalanine interaction. Employing MATI threshold ionization of indole benzene plus an appearance energy measurement of indole ${ }^{+}$, Neusser and co-workers determined the dissociation energy of indole benzene as $D_{0}=21.8 \pm 0.18 \mathrm{~kJ} / \mathrm{mol}^{33}$ and that of indole benzene- $d_{6}$ as $22.3 \pm 0.18 \mathrm{~kJ} / \mathrm{mol} .{ }^{29}$ These are among the most precise $D_{0} \mathrm{~s}$ reported to date. As discussed above, the interpretation of this value also requires the identification of the different structural isomers and of the most stable structure. In addition to the $\pi$-stacking 
and $\mathrm{CH} \cdots \pi$ interactions in (benzene) $)_{2}$ and (toluene $)_{2}$, indole benzene can also form an nonclassical NH $\cdots \pi$ hydrogen-bond. The 3D PES for parallel $\pi \cdots \pi$ structures has been calculated with the MP2 and SCS-MP2 methods and the aug-cc-pVDZ basis set, yielding two distinctly different minima structures for the two methods. Both structures are found to be weaker than the NH $\cdots \pi$ conformer at the SCS-CCSD(T)/CBS level of theory by more than $4 \mathrm{~kJ} / \mathrm{mol}$ and therefore are not expected to be observed in the supersonic jet. ${ }^{253}$ Due to the large positive electrostatic potential of the NH hydrogen, the interaction energy $\Delta E_{2}$ of the T-shaped $\mathrm{NH} \cdots \pi$ bonded conformer is nearly $5 \mathrm{~kJ} / \mathrm{mol}$ larger in magnitude than that of the $\mathrm{CH} \cdots \pi$ bonded conformers at the SCSCCSD[T]/CBS level of theory. ${ }^{253}$ The calculated interaction energy $\Delta E_{2}=-22.9 \mathrm{~kJ} / \mathrm{mol}$ of this conformer is slightly larger in magnitude than the measured $D_{0}$. However interaction energies are expected to be larger than the dissociation energy $D_{0}$, as discussed in Section 1 . On the other hand, Braun et al. calculated a dissociation energy $D_{0}=22.2 \mathrm{~kJ} / \mathrm{mol}$ at the MP2/CBS $+\Delta \mathrm{CCSD}[\mathrm{T}] / 6-$ $31 \mathrm{G}^{* *}(0.25,0.15)+\Delta \mathrm{ZPVE}\left(\mathrm{MP} 2 / 6-31 \mathrm{G}^{*}\right)$ level of theory for the $\mathrm{NH} \cdots \pi$ hydrogen-bonded Tshaped structure. ${ }^{29}$

Based on the excellent agreement between measured and calculated dissociation energies, this structure was assigned to be the observed complex. However, the structure was contested by Biswal and co-workers, ${ }^{254}$ who measured the supersonic jet IR spectrum in the NH stretch region of the indole moiety and compared it to the results of dispersion-corrected B97-D/TZVPP calculations. They found an additional T-shaped local minimum, labeled T', with the plane of the indole moiety tilted $\approx 60^{\circ}$ from that of benzene. The dissociation energies of the $\mathrm{T}$ - and $\mathrm{T}$ '-shaped isomers are very close (20.4 and $20.3 \mathrm{~kJ} / \mathrm{mol}$, respectively, including counterpoise corrections), indicating a very flat potential along the coordinate that interconverts the two conformers. Due to the better agreement of the experimental NH stretch frequency $\left(3479 \mathrm{~cm}^{-1}\right)$ with that calculated for the T'shaped isomer $\left(3487 \mathrm{~cm}^{-1}\right)$ than with the T-shaped structure $\left(3498 \mathrm{~cm}^{-1}\right)$, Biswal et al. concluded that the T' structure is observed. ${ }^{254}$ Summarizing, one concluded that even relatively strong and strongly directional $\mathrm{NH} \cdots \pi$ interactions may lead to two nearly degenerate isomers. In such cases, the comparison of calculated to the experimental dissociation energies must be made with great 
care. $^{254}$

\subsubsection{Complexes with Anisole}

In anisole, both the methoxy group and the $\pi$-electrons plane can act as H-bond acceptor sites. In addition to dispersive and $\mathrm{OH} \cdots \pi$ interactions that occur in complexes with benzene, $\mathrm{OH} \cdots \mathrm{O}$ hydrogen bonds can now also come into play. The subtle competition between the different possible interactions are the subject of recent spectroscopic studies in supersonic jets. ${ }^{255-259}$ For anisole.water ${ }^{256,257}$ and anisole.methanol ${ }^{259}$ it has been found that complexes with an $\mathrm{OH} \cdots \mathrm{O}$ hydrogen bond are more abundant than structures dominated by purely dispersive or $\mathrm{OH} \cdots \pi$ interactions. In contrast to these findings, the experimental rotational constants of anisole $\cdot \mathrm{NH}_{3}$ measured with high resolution LIF spectroscopy (laser induced fluorescence) fit best for a structure in which ammonia forms an $\mathrm{NH} \cdots \pi$ hydrogen bond to the anisole moiety. ${ }^{258,260}$ A comparison of the calculated binding energies of the $\mathrm{NH} \cdots \pi$ and $\mathrm{N}-\mathrm{H} \cdots \mathrm{O}$ complexes yields an unclear picture: depending on the method for the dissociation energy and structure calculation, either the $\mathrm{N}-\mathrm{H} \cdots \pi$ or the $\mathrm{N}-\mathrm{H} \cdots \mathrm{O}$ complex is expected to be more strongly bound.

The $D_{0}$ of anisole·benzene has been measured by dispersed fluorescence (see Section 3.1). ${ }^{255}$ The $D_{0}$ of the $S_{1}$ state was bracketed between 20.5 and $22.2 \mathrm{~kJ} / \mathrm{mol}$. Subtracting the redshift of $\delta v=354 \mathrm{~cm}^{-1}(4.2 \mathrm{~kJ} / \mathrm{mol})$ leads to a ground state $D_{0}=16.3-18.0 \mathrm{~kJ} / \mathrm{mol}$.

(Anisole) $)_{2}$ is a $\pi$-stacked centrosymmetric $\left(C_{i}\right)$ dimer. ${ }^{261}$ Due to symmetry restrictions, the $S_{1}\left(A_{g}\right)$ state cannot be excited by one-photon excitation, the lowest state that can be optically excited is the $S_{2}\left(A_{u}\right)$ state. An excitonic splitting between the $S_{1}$ and $S_{2}$ states occurs which has been measured to be $14 \mathrm{~cm}^{-1} .{ }^{262}$ The dissociation energy has been determined using VMI (velocity mapping ion imaging) in $2013,{ }^{36}$ however, the measured ground-state $D_{0}\left(S_{0}\right)$ of $47.0 \mathrm{~kJ} / \mathrm{mol}$ was in stark contrast to the calculated DFT (M05-2X/aug-cc-pVTZ) binding energy of $17.7 \mathrm{~kJ} / \mathrm{mol}{ }^{258}$ The experimental $D_{0}$ has recently been reinvestigated applying a two-color appearance-energy scheme (2C-AE). The revised dissociation energies are $21.5 \pm 1.2$ in the $S_{0}$ state and $48.1 \pm 2.4 \mathrm{~kJ} / \mathrm{mol}$ in the ion ground state. The neutral ground-state $D_{0}$ is now in good agreement with the DFT-D3 
zero-point-corrected $\operatorname{CCSD}(\mathrm{T}) / \mathrm{CBS}$ dissociation energy $D_{0}=21.1 \pm 0.2 \mathrm{~kJ} / \mathrm{mol} .^{37}$

\section{Summary and Outlook}

Above, we have discussed experimental and theoretical methods for measuring and calculating accurate dissociation energies $D_{0}$ of dispersively bound $\mathrm{M} \cdot \mathrm{S}$ complexes, where $\mathrm{M}$ is an aromatic or heteroaromatic molecule and S is a solvent molecule. In Section 2, we propose a classification of dispersively bound $\mathrm{M} \cdot \mathrm{S}$ complexes according to the frontier orbitals of $\mathrm{M}$ and $\mathrm{S}$ (with $\mathrm{M}_{2}$, that is, $\mathrm{M}=\mathrm{S}$ as a special case): As type-I we denote complexes in which the HOMO and LUMO of $\mathrm{M}$ and $\mathrm{S}$ are not $\pi$-electron orbitals, as type-II we classify complexes in which the HOMO and LUMO of M are both $\pi$-electron orbitals, but those of S are not, and as type-III we classify M.S complexes in which the HOMO and LUMO of both $\mathrm{M}$ and S are $\pi$-electron orbitals.

With regard to type-II complexes, we discussed in Sections 2 and 3 that the dissociation energy can be determined in either the $S_{0}$ ground state of $\mathrm{M} \cdot \mathrm{S}$, the $S_{1}$ excited state $\mathrm{M}^{*} \cdot \mathrm{S}$ or the ion ground state $\mathrm{M}^{+} \cdot \mathrm{S}$, giving $D_{0}(\mathrm{M} \cdot \mathrm{S}), D_{0}\left(\mathrm{M}^{*} \cdot \mathrm{S}\right)$ or $D_{0}\left(\mathrm{M}^{+} \cdot \mathrm{S}\right)$, respectively. The $S_{1}$ or ion ground state dissociation energies can be converted to $D_{0}(\mathrm{M} \cdot \mathrm{S})$ using a thermochemical cycle and further experimental information, that is, either the $S_{0} \rightarrow S_{1} 0_{0}^{0}$ excitation energy or the adiabatic ionization energy of M.

We point out in Sections 2, 3 and 5 that the electronic structure of type-I and type-III complexes gives rise to severe problems when determining the $D_{0}$ values via either the $S_{1}$ excited state or the ion ground state. The reason for this is that the change in electronic structure of M.S upon electronic excitation or ionization leads to major changes of the intermolecular potential energy surfaces of $\mathrm{M}^{*} \cdot \mathrm{S}$ or $\mathrm{M}^{+} \cdot \mathrm{S}$, relative to the ground state PES. If the minimum-energy geometries of the $S_{0}$ and $S_{1}$, or $S_{0}$ and ion states are sufficiently displaced, it becomes difficult or impossible to measure the $S_{0} \rightarrow S_{1} 0_{0}^{0}$ excitation energy or the adiabatic ionization energy of M.S. Then, the corresponding thermochemical cycle cannot be employed and $D_{0}\left(S_{0}\right)$ is not accessible. The intrinsic nature of these problems have not always been realized, sometimes leading to experimental pitfalls. 
Due to the recent improvements of theoretical methods for calculating intermolecular interactions and potential energy surfaces, the PESs of even large M.S complexes can now be computationally studied and potential problems diagnosed before the experiment.

Details of the experimental methods are discussed in Section 3: Most methods can be classified as predissociative, i.e., M.S is first cooled to its lowest $S_{0}$ state vibrational level and is then excited to a vibrational level that energetically lies above the dissociation energy $D_{0}$, so that vibrational predissociation (VP) can occur. The methods are categorized according to whether the vibrationally "hot" state that undergoes VP is (a) the $S_{0}$ state, (b) the $S_{1}$ excited state, or (c) the ion ground state $\mathrm{M}^{+}$.S. Predissociative methods on the ground state surface employing infrared excitation or Raman pumping can be employed for type-I and type-III complexes. For type-II complexes, stimulated-emission pumping, $S_{1}$-state or ionizative methods can be generally employed. Problems arise when type-II complexes approach type-III, as for example for heterodimers where M and $\mathrm{S}$ have similar electronic structure.

For noble-gas dimers (or heterodimers), which are type-I $\mathrm{M}_{2}$ or $\mathrm{M} \cdot \mathrm{X}$ complexes, $D_{0}$ can be obtained from the time-honored extrapolative method. This involves measuring vibronic or ionizative spectroscopic transitions associated with the (single) vibrational coordinate and extrapolating the vibrational energies of the $v^{\prime}, v^{\prime}+1, \ldots$ levels to the dissociation limit. If the functional shape of the potential-energy curve is known (often from theory), the parameters of the potential-energy curve can be determined from the frequency spacings between a series of vibrational or vibronic bands, if the vibrational quantum numbers $v^{\prime}$ are correctly established. This directly gives the well depth $D_{e}\left(S_{0}\right)$; via a calculation of the zero-point vibrational energy, $D_{0}$ is also obtained. Unfortunately, it is difficult to extend the spectroscopic-extrapolative method from diatomics to triatomic or larger $\mathrm{M} \cdot \mathrm{S}$ complexes, since high vibrational levels of multidimensional potential energy surfaces are anharmonically coupled, both between the intramolecular vibrational coordinates and between the intra- and intermolecular vibrational coordinates, and their energy differences do not follow simple extrapolation rules. In many cases, full variational calculations of the high-lying vibrational levels are prohibitively expensive. In such cases, predissociative methods on the ground state surface 
should be employed.

Today, quantum chemical computations of electronic binding energies $D_{e}$ can be performed with very high precision, rivalling or even surpassing the accuracy of experimental determinations of dissociation energies of dispersion-dominated aromatic molecular complexes. Computations at high levels of coupled-cluster theory may be performed with unprecedented accuracy for selected points on the relevant potential energy surfaces, using extrapolation techniques or explicitlycorrelated two-electron basis functions to approach the limit of a complete one-electron basis set. It may be particularly advantageous to combine various computational methods in a multilevel scheme. Computationally less demanding methods such as spin-component-scaled secondorder Møller-Plesset perturbation theory, dispersion-corrected density-functional theory, densityfunctional-based symmetry-adapted perturbation theory, or methods based on the random-phase approximation offer access to the computation of intermolecular potential energy surfaces and the computation of zero-point vibrational energies, which are required for a direct comparison between experimentally and theoretically determined dissociation energies.

Experimental and computational techniques have both reached a level of accuracy at which they can mutually enrich and reinforce their further methodological development.

\section{Acknowledgments}

We thank the Swiss National Science Foundation (SNSF) and the Deutsche Forschungsgemeinschaft (DFG) for financial support through the Priority Programme SPP 1807 “Control of London dispersion interactions in molecular chemistry" (grants DFG KL 721/5-1 and SNSF 200021E160404). 


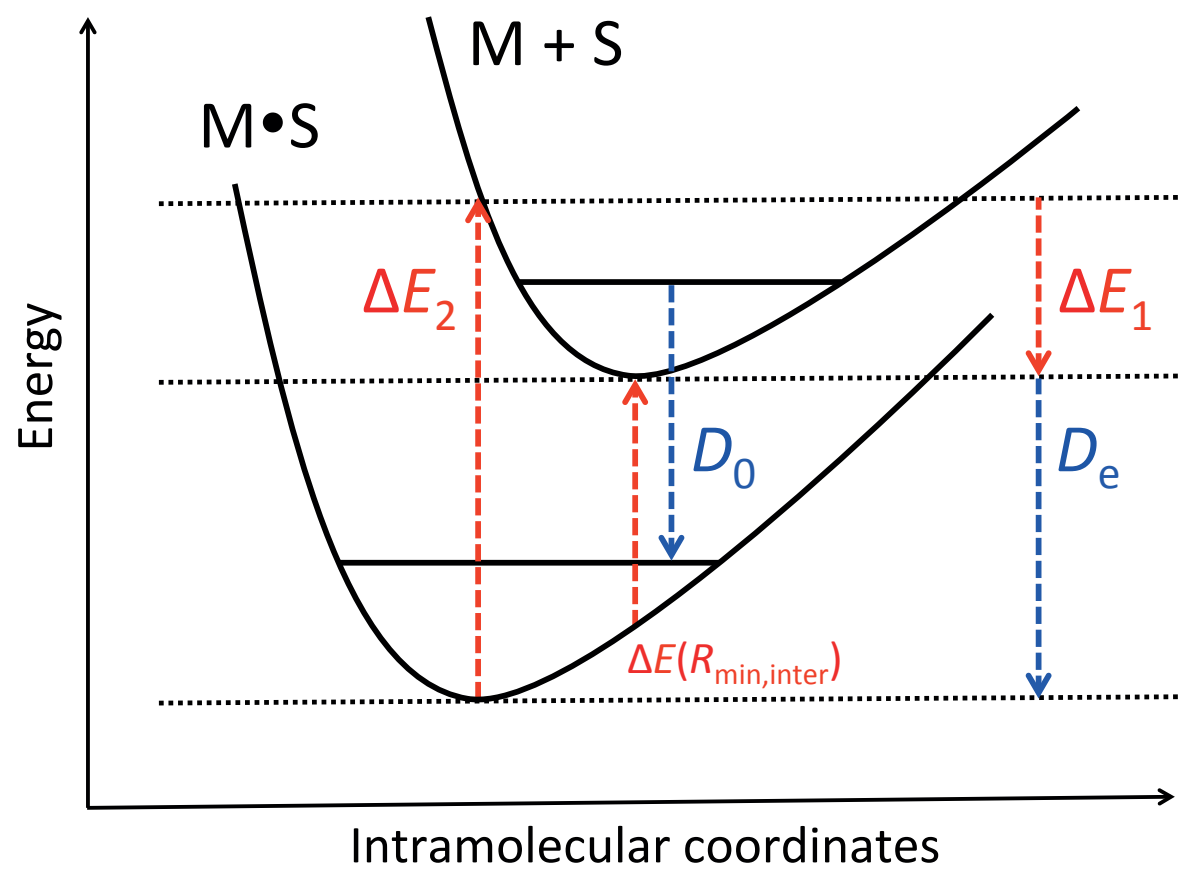

Figure 2: Illustration of the terminology and sign conventions used in the present review. Shown are the interaction energy $\Delta E_{2}=\Delta E\left(R_{\text {min,inter+intra }}\right)$, the electronic binding energy $D_{e}$, the dissociation energy $D_{0}$ and the fixed-fragments interaction energy $\Delta E\left(R_{\text {min,inter }}\right)$. Upgoing arrows refer to negative values, downgoing arrows refer to positive values. 


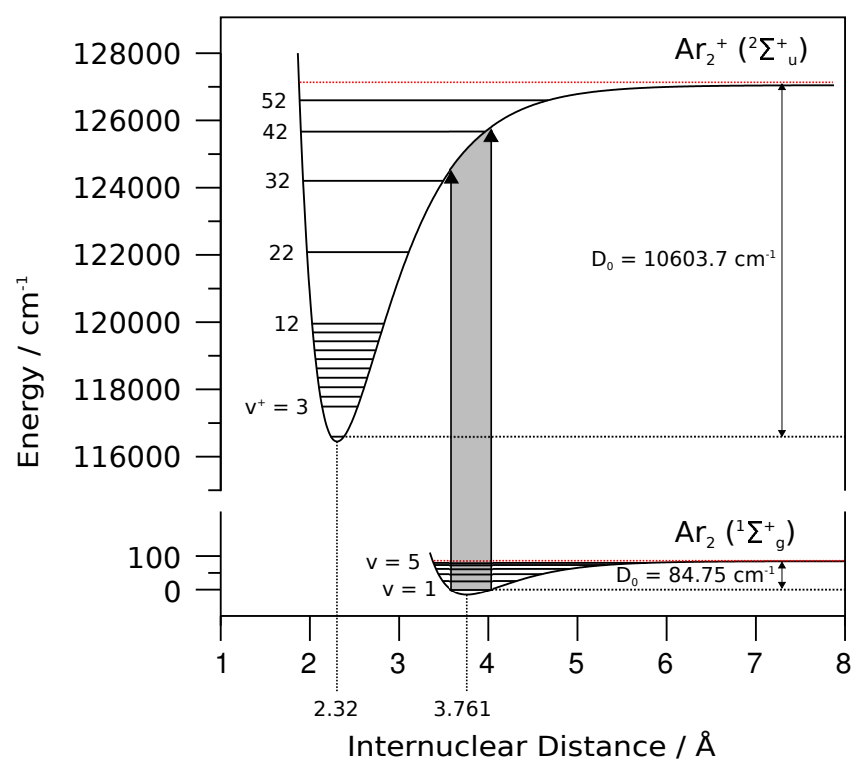

Figure 3: Neutral ${ }^{1} \Sigma_{g}^{+}$and ion ${ }^{2} \Sigma_{u}^{+}$ground state potentials of $\mathrm{Ar}_{2}$. The Franck-Condon window for ionization from the ${ }^{1} \Sigma_{g}^{+} v^{\prime \prime}=0$ vibration is indicated in grey. The energy axis of the ground state is magnified $10 \mathrm{x}$ with respect to the ion state. 


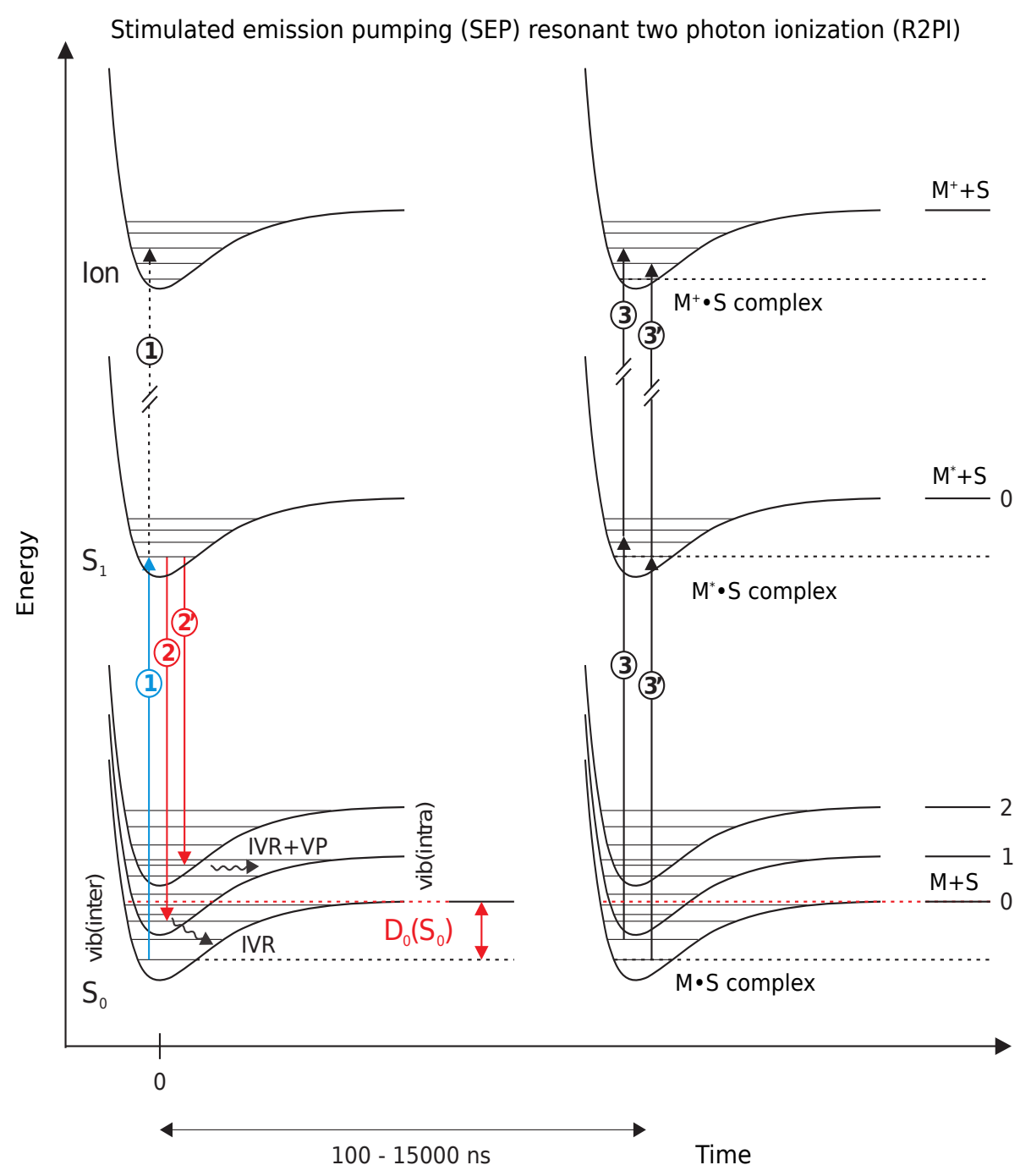

Figure 4: Schematic level diagram of the SEP-R2PI experiment, as applied to a type-II M-S complex. The potential energy curves indicate the $S_{0}$ state, $S_{1}$ excited-state and ion ground-state potentials plotted against the M $\cdots \mathrm{S}$ distance (intermolecular stretching coordinate). The intramolecular vibrational levels of $\mathrm{M}$ are indicated as horizontal bars connected to the vertically shifted Morsetype potentials, and are shown for the $S_{0}$ state only. The intermolecular stretching vibrational levels are plotted within the Morse potentials. The global horizontal axis indicates the time between the pump/dump steps and the two-photon ionization detection step, which is delayed by $100 \mathrm{~ns}$ to 15 $\mu \mathrm{s}$. 
Figure 5: Schematic level diagram of a type II M.S complex in its neutral and cationic ground states. Also indicated are the adiabatic ionization energy (AIE) of M.S and of M, as well as the appearance energy (AE) of the $\mathrm{M}^{+}$daughter ion. The scheme illustrates the thermochemical cycles $D_{0}+\mathrm{AIE}(\mathrm{M})=D_{0}^{+}+\mathrm{AIE}(\mathrm{M} \cdot \mathrm{S})$, as well as $D_{0}^{+}=\mathrm{AE}\left(\mathrm{M}^{+}\right)-\operatorname{AIE}(\mathrm{M} \cdot \mathrm{S})$. 
2

3

4

5

6

7

8

9

10

11

12

13

14

15

16

17

18

19

20

21

22

23

24

25

26

27

28

29

30

31

32

33

34

35

36

37

38

39

40

41

42

43

44

45

46

47

48

49

50

51

52

53

54

55

56

57

58

59

60

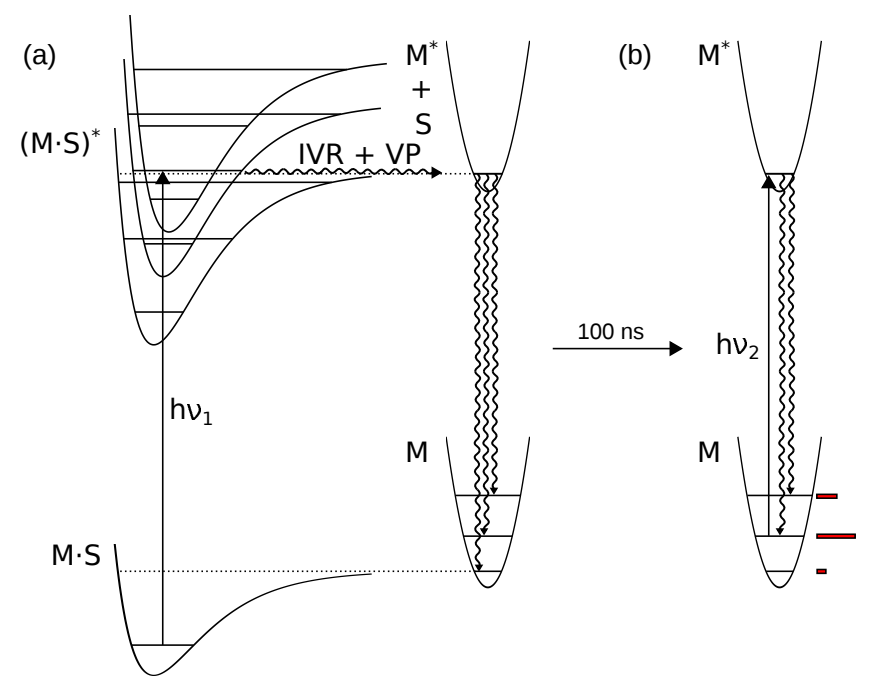

Figure 6: (a) Determination of the excited-state dissociation energy of an M.S complex by dispersed fluorescence. The $D_{0}$ of the ground state is then calculated using thermochemical cycles, see Figure 5.(b) A two-laser variant of the method described in ref. ${ }^{18}$, detection of the $\mathrm{M}^{*}$ dissociation product is performed on a "hot" level that is not populated in the cold supersonic jet environment. 


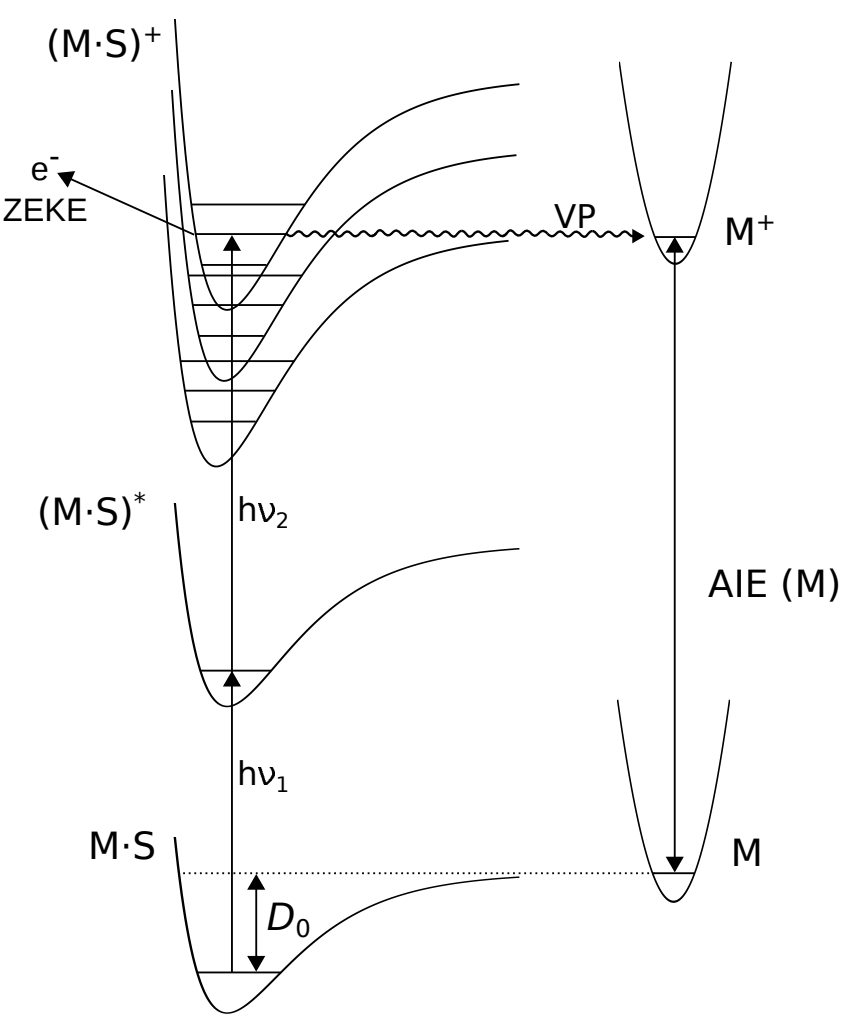

Figure 7: Determination of the ground-state dissociation energy $D_{0}$ of an M.S complex by dispersed appearance energy measurements, see section 3.2. Note that the energy of the departing photoelectron (not shown in the Figure9 is not measured but assumed to be zero near the (M.S) ${ }^{+}$ dissociation limit, which introduces considerable ambiguity in the measurement. 
1

2

3

4

5

6

7

8

10

11

12

13

14

15

16

17

18

19

20

21

22

23

24

25

26

27

28

29

30

31

32

33

34

35

36

37

38

39

40

41

42

43

44

45

46

47

48

49

50

51

52

53

54

55

56

57

58

59

60 (a)

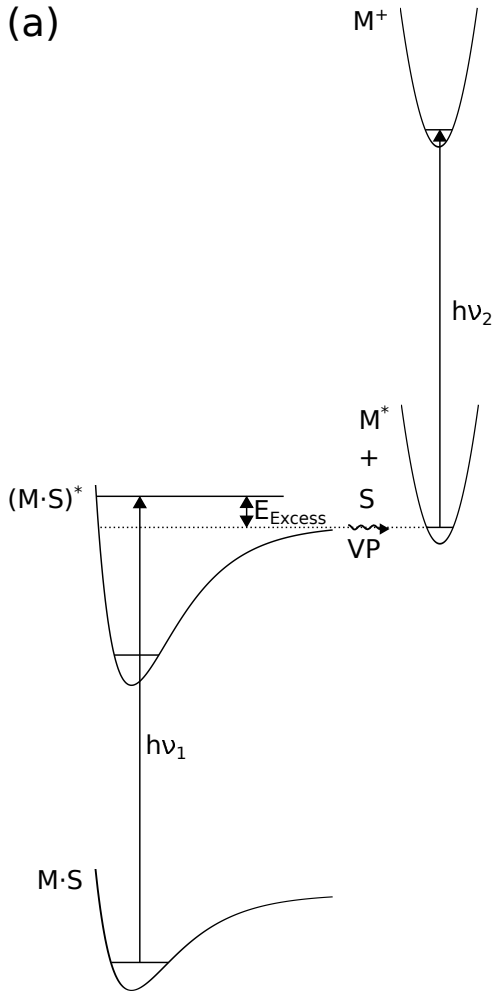

(b)

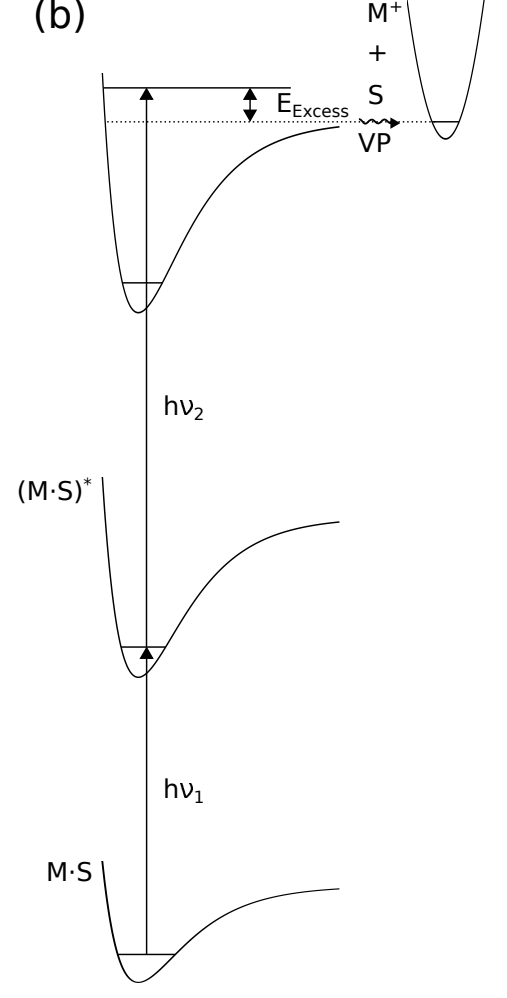

Figure 8: Determination of the dissociation energy in the (a) first excited or (b) ionic ground state using velocity map imaging (VMI). The excess energy $E_{\text {excess }}$ is assumed to correspond to the highest measured translational energy $E_{\text {trans }}$ of the detected ion fragment $\mathrm{M}^{+}$. The $D_{0}$ of the ground state is then calculated using thermochemical cycles, see Figure 5. 

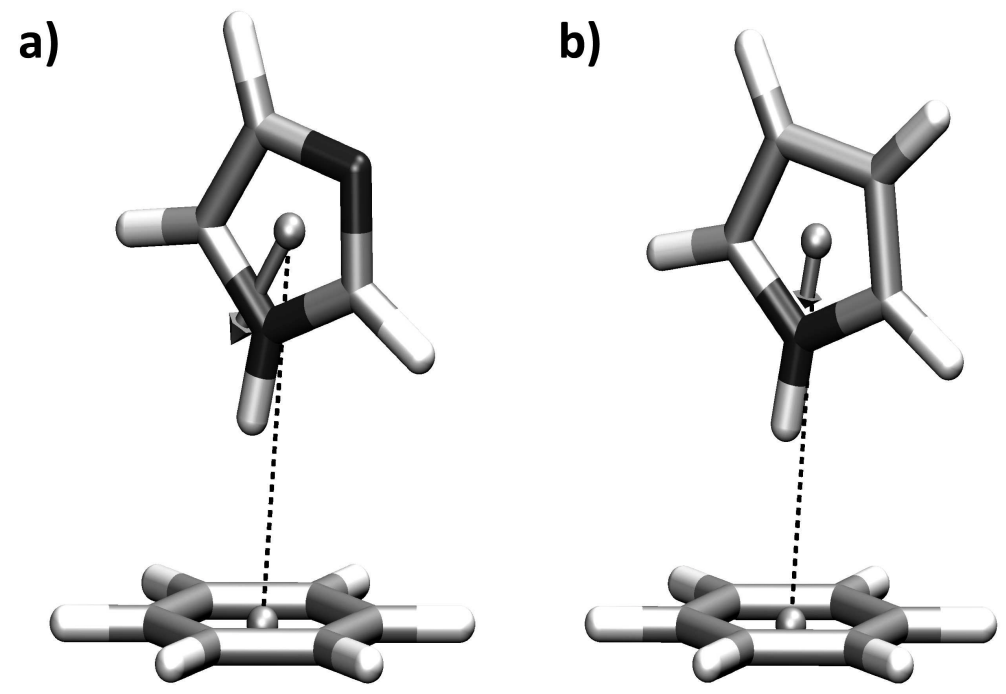

Figure 9: Optimized geometries of the (a) imidazole benzene and (b) pyrrole benzene complexes. $R_{1}=8.17 a_{0}\left(R_{2}=8.21 a_{0}\right)$ is the distance between the center-of-mass of the imidazole (pyrrole) molecule and the center-of-mass of the benzene molecule. The arrows represent the imidazole and pyrrole monomer dipoles $(2 \mu / e)$. Reprinted with permission from Ref. 110. Copyright 2014 Elsevier B.V. 
1

2

3

4

5

6

7

8

9

10

11

12

13

14

15

16

17

18

19

20

21

22

23

24

25

26

27

28

29

30

31

32

33

34

35

36

37

38

39

40

41

42

43

44

45

46

47

48

49

50

51

52

53

54

55

56

57

58

59

60
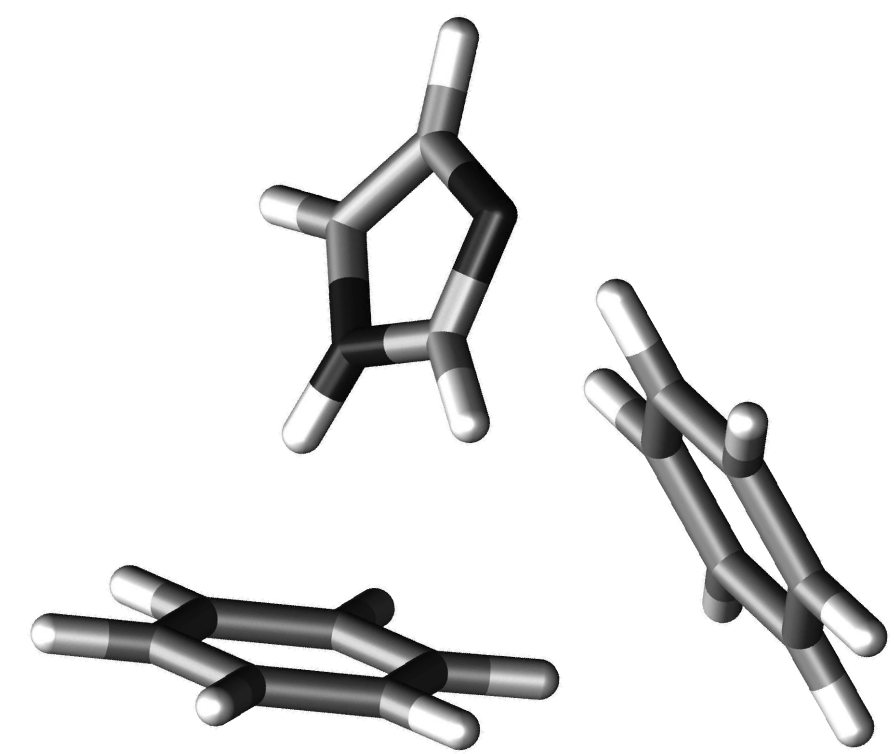

Figure 10: (Benzene) $)_{2}$ imidazole trimer, for which harmonic vibrational frequencies have been computed at the SCS-CC2/def2-QZVPP level in the $S_{1}$ state. 

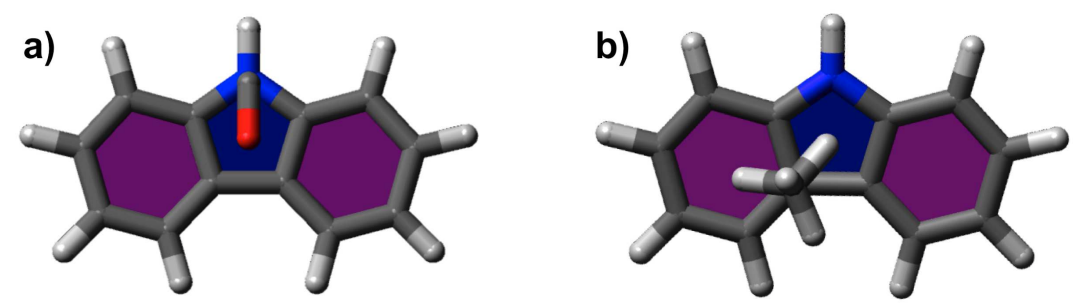

Figure 11: Optimized geometries of the (a) carbazole $\mathrm{CO}$ and (b) carbazole $\cdot \mathrm{CH}_{4}$ complexes obtained at the SCS-CC2/aug-cc-pVTZ level. 


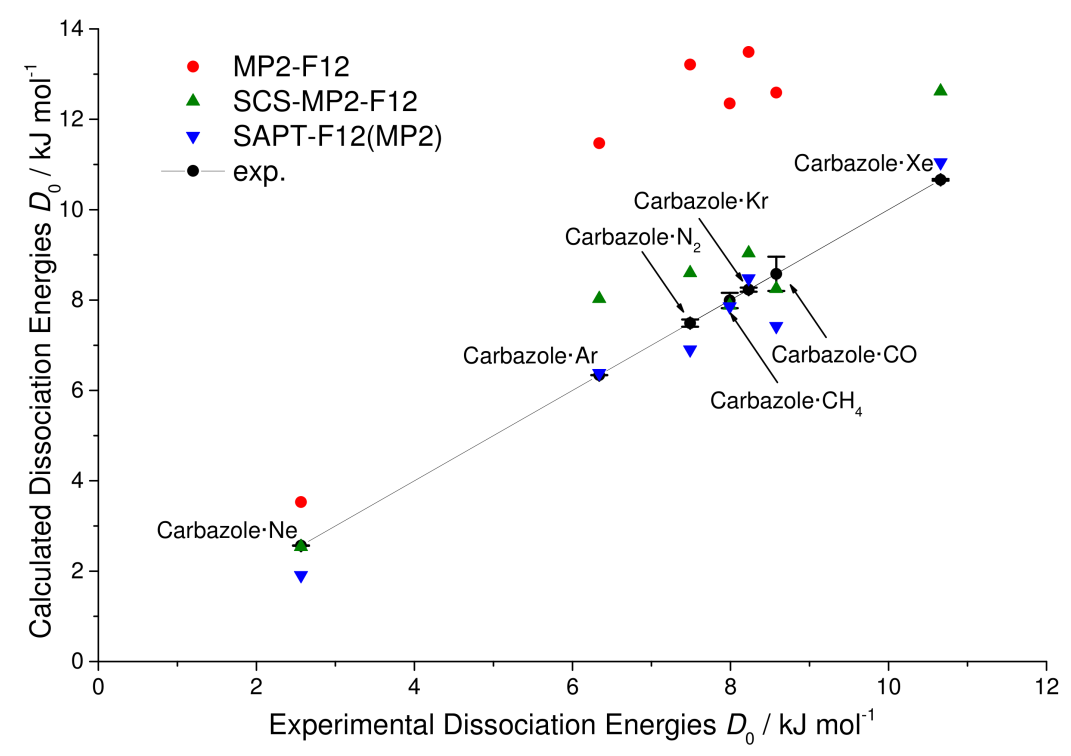

Figure 12: Comparison of experimental and calculated binding energies $D_{0}$ of carbazole.X complexes with $\mathrm{X}=\mathrm{Ne}, \mathrm{Ar}, \mathrm{Kr}, \mathrm{Xe}, \mathrm{CO}, \mathrm{N}_{2}$, and $\mathrm{CH}_{4}$. The MP2-F12 value for carbazole.Xe is 19.58 $\mathrm{kJ} / \mathrm{mol}$ and not shown. Harmonic ZPVE from PBE-D3/def2-TZVP computations. 


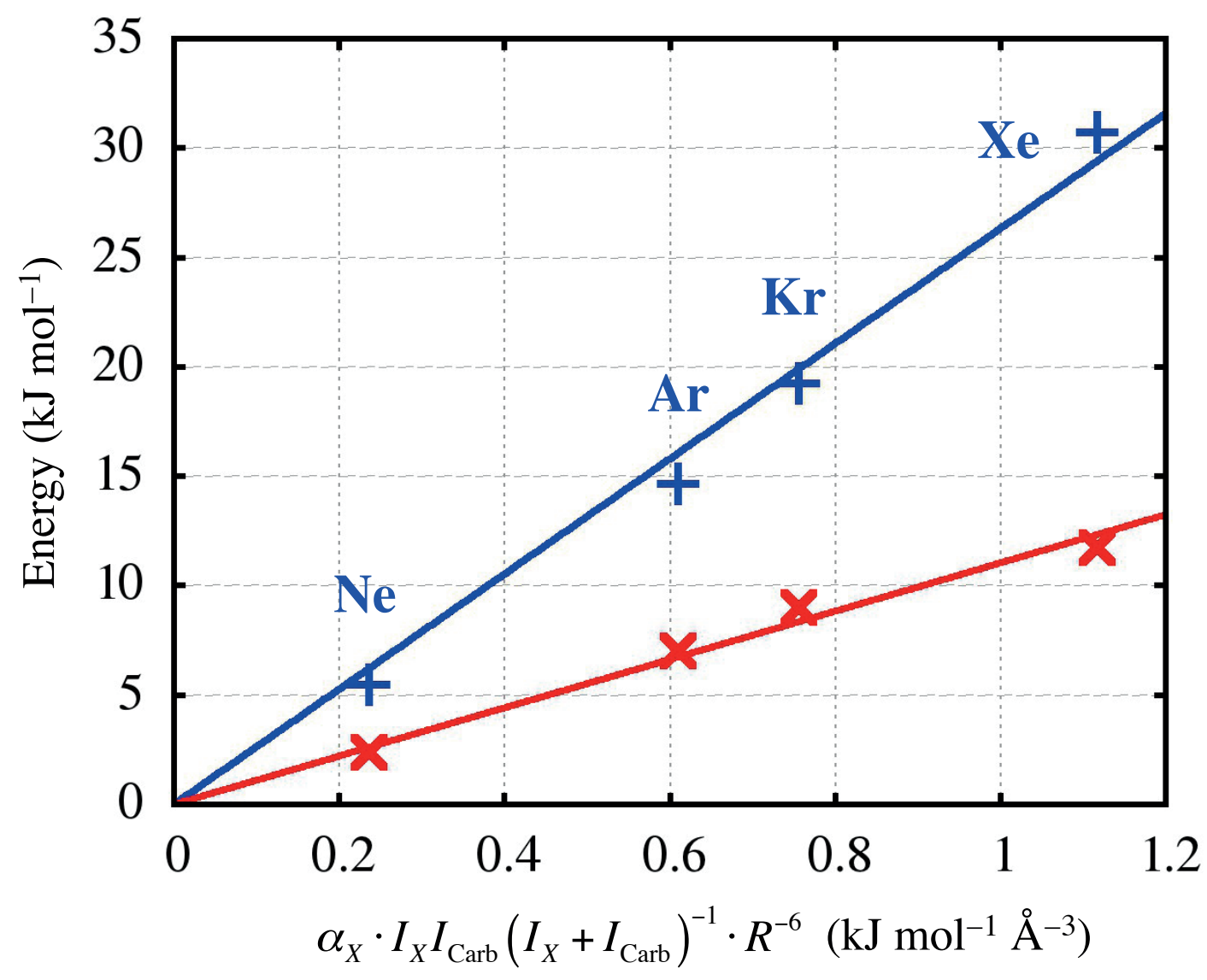

Figure 13: $E_{\mathrm{disp}}^{(2)}$ (blue points and line) and computed binding energy $D_{e}$ (red points and line) of the carbazole complexes with $\mathrm{X}=\mathrm{Ne}, \mathrm{Ar}, \mathrm{Kr}, \mathrm{Xe}$ as function of the static polarizability $\alpha_{\mathrm{X}}$. 
b)
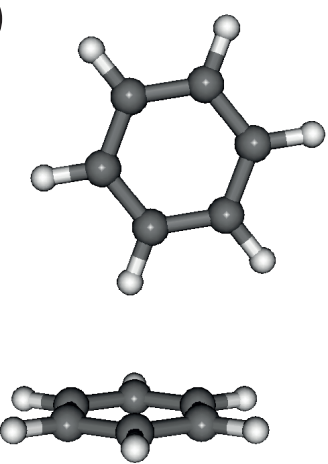

global minimum

$\mathrm{M} 2\left(\mathrm{C}_{\mathrm{s}}\right)$
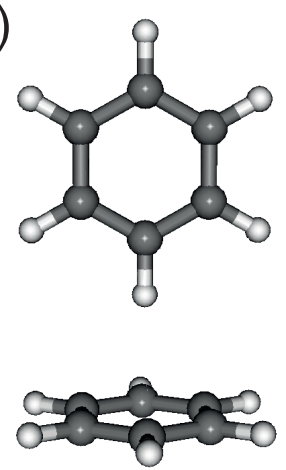

saddle point S3 ( $\left.\mathrm{C}_{2 \mathrm{v}}\right)$ c)
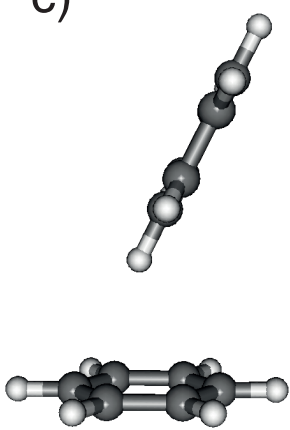

saddle point S3a $\left(\mathrm{C}_{\mathrm{s}}\right)$ d)
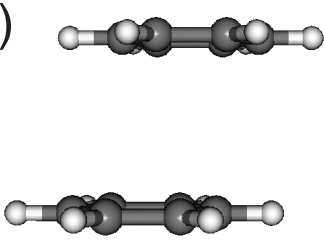

local minimum

$\mathrm{M} 1\left(\mathrm{C}_{2 h}\right)$ e)

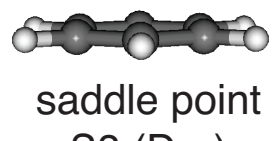

Figure 14: Neutral ground-state stationary points on the (benzene) $)_{2}$ DFT-SAPT intermolecular PES: ${ }^{233,234}$ (a) the tipped T-shaped global minimum M2, (b) the T-shaped $C_{2 v}$ saddle point S3, (c) the tilted T-shaped $C_{S}$ saddle point S3a, (d) the parallel-displaced $\left(C_{2 h}\right)$ local minimum M1, (e) the stacked-parallel $\left(D_{6 h}\right)$ saddle point S8. Nomenclature as defined in Refs. 233 and 234. 

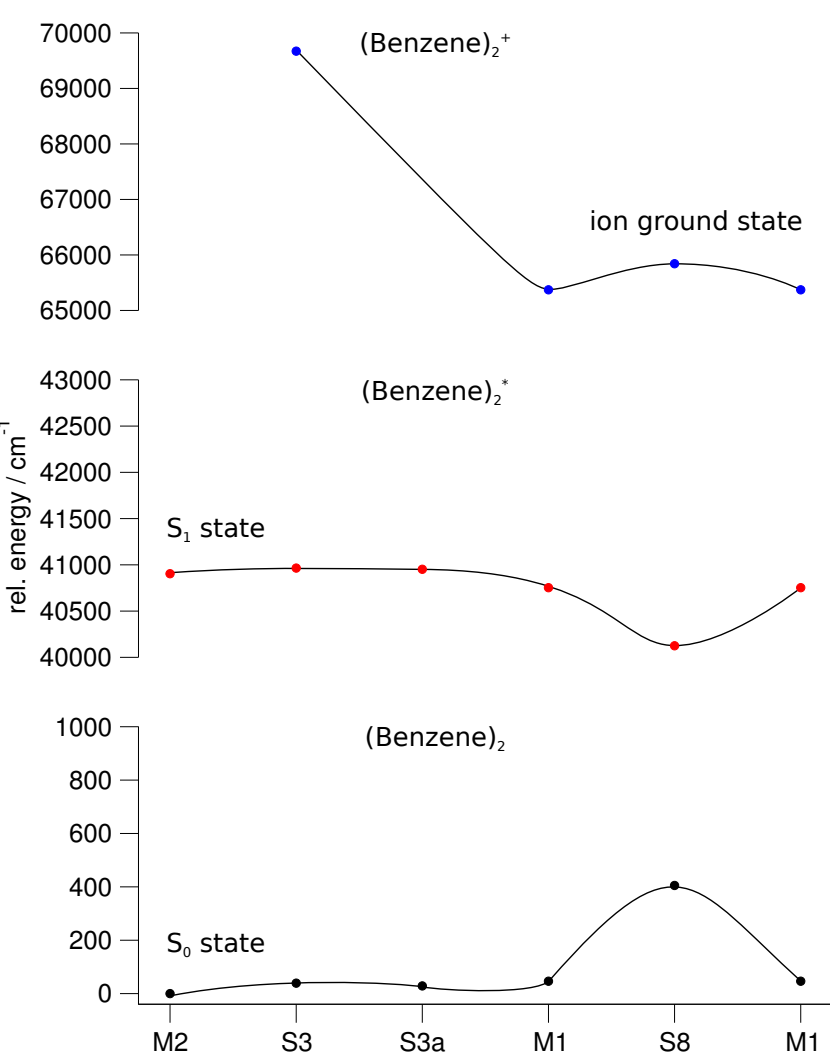

Figure 15: Calculated energies of (benzene) $)_{2}$ in the $S_{0}$ state, $S_{1}$ state and $\mathrm{Bz}_{2}^{+}$ion ground state energies for the different T-shaped and $\pi$-stacked conformers shown in Figure 14 . The energies of the different conformers are connected to indicate qualitative features of the $S_{0}$ (black), $S_{1}$ (red) and ion (blue) potential energy surfaces. The $S_{0}$ state energies of the M2, S3, S3a, M1 and S8 and $D_{e}\left(S_{0}\right)$ are from Ref. 234, the vertical SCS-CC2/aug-cc-pVTZ $S_{1}$ energies are from Ref. 263, the vertical and adiabatic EOM-CCSD energies are from Ref. 245. 
Table 1: Dissociation energies $D_{0}$ (in $\mathrm{kJ} / \mathrm{mol}$ ) of neutral type I M.S complexes measured by pulsed-field ionization zero electronic kinetic energy (PFI-ZEKE) spectroscopy, photofragment angular distributions (PHOFAD), position-sensitive translational spectroscopy (POSTS), Doppler spectroscopy and velocity map imaging (VMI).

\begin{tabular}{|c|c|c|c|c|c|}
\hline Complex & Method & Neutral $D_{0}$ & Calc. $D_{e}$ & Calc. $D_{0}$ & Refs. \\
\hline$(\mathrm{Ar})_{2}$ & PFI-ZEKE & 1.03 & & & 203 \\
\hline $\mathrm{I}_{2} \cdot \mathrm{Ar}$ T-shaped & VMI & $2.88 \pm 0.04$ & & & 264 \\
\hline $\mathrm{I}_{2} \cdot \mathrm{Ar}$ linear & VMI & $2.99 \pm 0.03$ & & & 264 \\
\hline$\left(\mathrm{H}_{2} \mathrm{O}\right)_{2}$ & VMI & $13.2 \pm 0.11$ & 20.8 & 13.2 & 211,213 \\
\hline$\left(\mathrm{D}_{2} \mathrm{O}\right)_{2}$ & VMI & $14.9 \pm 0.12$ & 20.8 & $14.9 \pm 0.06$ & 212,214 \\
\hline $\mathrm{H}_{2} \mathrm{O} \cdot \mathrm{NH}_{3}$ & VMI & $18.4 \pm 0.12$ & 27.0 & 17.6 & 265,266 \\
\hline$\left(\mathrm{NH}_{3}\right)_{2}$ & VMI & $7.90 \pm 0.24$ & 13.8 & 7.91 & 217,267 \\
\hline$(\mathrm{HCl})_{2}$ & POSTS & $5.25 \pm 0.01$ & & & 268 \\
\hline$(\mathrm{HCl})_{2}$ & PHOFAD & $5.25 \pm 0.1$ & 8.30 & 5.09 & 269 \\
\hline $\mathrm{H}_{2} \mathrm{O} \cdot \mathrm{HCl}$ & VMI & $15.96 \pm 0.12$ & 23.01 & 16.12 & 216,270 \\
\hline$(\mathrm{HF})_{2}$ & PHOFAD & $12.70 \pm 0.02$ & 19.1 & 12.7 & 271,272 \\
\hline$H F \cdot D F$ & PHOFAD & $12.94 \pm 0.02$ & 19.1 & 12.9 & 273,274 \\
\hline $\mathrm{DF} \cdot \mathrm{HF}$ & PHOFAD & $13.84 \pm 0.02$ & 19.1 & 13.8 & 273,274 \\
\hline $\mathrm{C}_{2} \mathrm{H}_{2} \cdot \mathrm{HF}$ & PHOFAD & $13.0 \pm 0.02$ & & & 275 \\
\hline $\mathrm{C}_{2} \mathrm{H}_{2} \cdot \mathrm{HCl}$ & PHOFAD & $9.93 \pm 0.07$ & & & 276 \\
\hline $\mathrm{C}_{2} \mathrm{H}_{2} \cdot \mathrm{HCl}$ & VMI & 8.37 & & & 277 \\
\hline $\mathrm{C}_{2} \mathrm{H}_{2} \cdot \mathrm{NH}_{3}$ & VMI & 10.8 & & & 278 \\
\hline ortho- $\mathrm{D}_{2} \cdot \mathrm{HF}$ & PHOFAD & $0.61 \pm 0.02$ & & & 279 \\
\hline para- $\mathrm{D}_{2} \cdot \mathrm{HF}$ & PHOFAD & $0.81 \pm 0.02$ & & & 279 \\
\hline $\mathrm{N}_{2} \cdot \mathrm{HF}$ & PHOFAD & $4.76 \pm 0.02$ & 9.12 & 4.98 & 280,281 \\
\hline $\mathrm{CO}_{2} \cdot \mathrm{HF}$ & PHOFAD & $8.04 \pm 0.5$ & & & 282 \\
\hline $\mathrm{HCN} \cdot \mathrm{HF}$ & PHOFAD & $23.6 \pm 0.12$ & & & 283 \\
\hline $\mathrm{NO} \cdot \mathrm{HF}$ & Doppler & 5.36 & & & 284 \\
\hline$(\mathrm{NO})_{2}$ & Doppler & $8.49 \pm 0.48$ & & & 285 \\
\hline $\mathrm{NO} \cdot \mathrm{CH}_{4}$ & VMI & $1.23 \pm 0.02$ & & & 286 \\
\hline
\end{tabular}


Table 2: Selected ion and neutral dissociation energies $D_{0}$ (in $\mathrm{kJ} / \mathrm{mol}$ ) of dispersively bound typeII $\mathrm{M} \cdot \mathrm{X}$ complexes with $\mathrm{X}=\mathrm{Ar}, \mathrm{Kr}$, measured by mass-analyzed threshold ionization (MATI), ion breakdown measurements (BM) two-color appearance potential (2C-AP), dispersed fluorescence (DIS) and velocity-map imaging (VMI).

\begin{tabular}{|c|c|c|c|c|c|c|}
\hline Complex & Method & Ion $D_{0}^{+}$ & Neutral $D_{0}$ & Calc. $D_{e}$ & Calc. $D_{0}$ & Refs. \\
\hline Benzene.Ar & $\mathrm{BM}$ & $3.7 \pm 0.6$ & $1.6 \pm 0.5$ & & & 32 \\
\hline Benzene.Ar & MATI & $<6.1$ & $<4.1$ & 4.62 & & $26,221,287$ \\
\hline Benzene.Ar & VMI & $5.82 \pm 0.06$ & $3.77 \pm 0.08$ & & & 34 \\
\hline Benzene $\cdot \mathrm{Kr}$ & MATI & $<7.6$ & $<4.8$ & $5.80-$ & - & $26,287,288$ \\
\hline s-Tetrazine $\cdot A r$ & DIS & & $3.0-4.3$ & $4.9-5.0$ & & 15,289 \\
\hline Fluorobenzene.Ar & MATI & 6.5 & 3.84 & 4.68 & 4.05 & 30,290 \\
\hline$p$-Difluorobenzene.Ar & VMI & $6.84 \pm 0.07$ & $4.03 \pm 0.05$ & 4.77 & 4.17 & $16,17,34,195$ \\
\hline$p$-Difluorobenzene.Ar & DIS & & $1.91-2.54$ & 4.81 & 4.20 & 291,292 \\
\hline$p$-Difluorobenzene $\cdot \mathrm{Kr}$ & VMI & $8.61 \pm 0.07$ & $4.75 \pm 0.08$ & & & 17 \\
\hline Phenylacetylene.Ar & - & & & 5.01 & 4.40 & 194 \\
\hline Styrene.Ar & $\mathrm{R} 2 \mathrm{PI}$ & & $4.7 \pm 0.2$ & & & 293 \\
\hline$p$-Xylene.Ar & $2 \mathrm{C}-\mathrm{AP}$ & & 1.4 & & & 294 \\
\hline Phenol-Ar & MATI & $6.40 \pm 0.06$ & $4.35 \pm 0.16$ & 5.19 & 4.65 & 295 \\
\hline Phenol·Ar & & & & 5.02 & 4.44 & 296 \\
\hline$p$-Fluorotoluene $\cdot \mathrm{Ar}$ & MATI & $6.10 \pm 0.24$ & $3.94 \pm 0.24$ & & & 297 \\
\hline Anisole $\cdot A r$ & MATI & $6.95 \pm 0.06$ & $4.61 \pm 0.06$ & 5.48 & 4.64 & 298 \\
\hline Indole $\cdot A r$ & MATI & $6.4 \pm 0.1$ & $5.4 \pm 0.2$ & & & 33 \\
\hline Indole.Ar & DIS & & $<6.5$ & & & 299 \\
\hline 1-Methylindole.Ar & DIS & & $5.4-7.8$ & & & 300 \\
\hline Dibenzofuran $\cdot A r$ & MATI & $<7.6$ & $<6.2$ & & & 31 \\
\hline Dibenzo- $p$-dioxin $\cdot A r$ & MATI & $8.8 \pm 0.1$ & $6.3 \pm 0.2$ & & & 31 \\
\hline Dibenzo- $p$-dioxin $\cdot A r$ & MATI & 8.58 & 6.04 & & & 301 \\
\hline Dibenzo- $p$-dioxin $\cdot \mathrm{Kr}$ & MATI & 10.98 & 7.66 & & & 301 \\
\hline$o$-Dichlorobenzene $\cdot \mathrm{Ar}$ & MATI & $6.04-7.85$ & $3.48-5.29$ & & & 302 \\
\hline$m$-Dichlorobenzene.Ar & MATI & $4.83-7.81$ & $2.65-5.63$ & & & 302 \\
\hline$p$-Dichlorobenzene $\cdot \mathrm{Ar}$ & MATI & $6.90-7.85$ & $4.90-5.85$ & & & 302 \\
\hline
\end{tabular}


Table 3: Dissociation energies $D_{0}$ of dispersively bound type-II $\mathrm{M} \cdot \mathrm{X}$ and $\mathrm{M} \cdot \mathrm{S}$ complexes, measured by the stimulated-emission pumping/resonant two-photon ionization (SEP-R2PI) method, see Figure 4.

\begin{tabular}{|c|c|c|}
\hline Complex & $D_{0}(\mathrm{~kJ} / \mathrm{mol})$ & Refs. \\
\hline Carbazole $\cdot \mathrm{Ne}$ & $2.565 \pm 0.004$ & 38 \\
\hline Carbazole $\cdot A r$ & $6.339 \pm 0.0017$ & 38,43 \\
\hline Carbazole $\cdot \mathrm{Kr}$ & $8.230 \pm 0.046$ & 38 \\
\hline Carbazole $\cdot \mathrm{Xe}$ & $10.66 \pm 0.02$ & 38 \\
\hline Carbazole $\cdot \mathrm{N}_{2}$ & $7.49 \pm 0.08$ & 39 \\
\hline Carbazole $\cdot \mathrm{CO}$ & $8.58 \pm 0.38$ & 39 \\
\hline Carbazole $\cdot \mathrm{CH}_{4}$ & $7.99 \pm 0.17$ & 39 \\
\hline 1-Naphthol·benzene & $21.21 \pm 0.25$ & 41 \\
\hline 1-Naphthol-benzene- $d_{6}$ & $21.25 \pm 0.25$ & 41 \\
\hline 1-Naphthol-cyclohexane & $28.95 \pm 0.13$ & 41 \\
\hline
\end{tabular}


Table 4: PBE0AC-SAPT results $(\mathrm{kJ} / \mathrm{mol})$ obtained at the $\operatorname{CCSD}(\mathrm{F} 12)+\left(\mathrm{T}^{*}\right)$ optimized geometries of the ImBz and PyBz complexes (see Ref. 110 for details). The reference values are $\Delta E_{2}=-22.8$ $(\mathrm{ImBz})$ and $-20.5 \mathrm{~kJ} / \mathrm{mol}(\mathrm{PyBz})$. Data taken from Ref. 110.

\begin{tabular}{|c|c|c|c|c|c|c|}
\hline \multirow{2}{*}{$\begin{array}{l}\text { Contribution }^{a} \\
\mathrm{~A}=\mathrm{Im}, \mathrm{Py}\end{array}$} & \multicolumn{2}{|c|}{ aug-cc-pVDZ } & \multicolumn{2}{|c|}{ aug-cc-pVTZ } & \multicolumn{2}{|c|}{ aug-cc-pV(DT) $Z^{b}$} \\
\hline & $\mathrm{ImBz}$ & $\mathrm{PyBz}$ & $\operatorname{ImBz}$ & $\mathrm{PyBz}$ & $\mathrm{ImBz}$ & $\mathrm{PyBz}$ \\
\hline$E_{\mathrm{pol}}^{(1)}$ & -17.25 & -14.94 & -17.34 & -15.05 & & \\
\hline$E_{\text {exch }}^{(1)}$ & 23.22 & 21.69 & 23.14 & 21.63 & & \\
\hline$E_{\text {ind }}^{(2)}(\mathrm{Bz} \rightarrow \mathrm{A})$ & -2.82 & -2.53 & -2.99 & -2.68 & & \\
\hline$E_{\text {ind }}^{(2)}(\mathrm{A} \rightarrow \mathrm{Bz})$ & -7.59 & -6.17 & -7.71 & -6.34 & & \\
\hline$E_{\text {exch-ind }}^{(2)}(\mathrm{A} \leftarrow \mathrm{Bz})$ & 2.49 & 2.18 & 2.66 & 2.33 & & \\
\hline$E_{\text {exch-ind }}^{(2)}(\mathrm{Bz} \leftarrow \mathrm{A})$ & 3.74 & 3.22 & 3.81 & 3.33 & & \\
\hline$E_{\text {disp }}^{(2)}$ & -26.33 & -25.53 & -24.84 & -24.39 & -24.32 & -24.01 \\
\hline$E_{\text {exch-disp }}^{(2)}$ & 3.24 & 3.08 & 3.13 & 3.02 & 3.10 & 3.00 \\
\hline$\delta(\mathrm{HF})$ & -2.89 & -2.53 & -2.95 & -2.61 & & \\
\hline Total $\left(\Delta E_{2}\right)$ & -24.18 & -21.54 & -23.08 & -20.74 & & \\
\hline
\end{tabular}

a) $E_{\text {disp }}^{(2)}$ and $E_{\text {exch-disp }}^{(2)}$ have been scaled by the ratio of the corresponding $\operatorname{CCSD}(\mathrm{F} 12)+\left(\mathrm{T}^{*}\right)$ and $\operatorname{CCSD}(\mathrm{T})$ electron-correlation contributions to the counterpoise-corrected interaction energy $\Delta E_{2}^{\mathrm{cp}}$.

b) Two-point $X^{-3}$ extrapolation from the aug-cc-pVDZ and aug-cc-pVTZ energies. 
Table 5: DFT-D3(BJ) results. Interaction energy $\left(\Delta E_{2}\right.$ in $\mathrm{kJ} / \mathrm{mol}$, with and without counterpoise correction) of the ImBz and PyBz complexes at their $\operatorname{CCSD}(\mathrm{F} 12)+\left(\mathrm{T}^{*}\right)$ optimized geometries. All results were obtained in the aug-cc-pVTZ basis. The reference values are $\Delta E_{2}=-22.8(\operatorname{ImBz})$ and $-20.5 \mathrm{~kJ} / \mathrm{mol}$ (PyBz). Data taken from Ref. 110.

\begin{tabular}{lrrrrr}
\hline \hline \multirow{2}{*}{ Method } & \multicolumn{2}{c}{ ImBz } & & \multicolumn{2}{c}{ PyBz } \\
\cline { 2 - 3 } \cline { 6 - 6 } PBE & w/o CP & w/CP & & w/o CP & w/CP \\
PBE0 & -23.08 & -22.24 & & -20.99 & -20.16 \\
TPSS & -24.35 & -23.51 & & -21.91 & -21.08 \\
TPSSh & -22.77 & -21.93 & & -20.67 & -19.86 \\
B2PLYP & -23.46 & -22.59 & & -21.23 & -20.39 \\
B2PLYP-F12 & -27.31 & -22.25 & & -25.00 & -19.98 \\
Frozen-core-B2PLYP & -26.18 & -22.52 & & -23.84 & -20.22 \\
Frozen-core-B2PLYP-F12 & -24.66 & -22.21 & & -22.40 & -19.95 \\
\hline \hline
\end{tabular}


Table 6: RPA results. Interaction energy ( $\Delta E_{2}$ in $\mathrm{kJ} / \mathrm{mol}$, with and without counterpoise correction) of the ImBz and PyBz complexes at their $\operatorname{CCSD}(\mathrm{F} 12)+\left(\mathrm{T}^{*}\right)$ optimized geometries. All results were obtained in the aug-cc-pVTZ basis. The reference values are $\Delta E_{2}=-22.8(\mathrm{ImBz})$ and -20.5 $\mathrm{kJ} / \mathrm{mol}(\mathrm{PyBz})$. Data taken from Ref. 110.

\begin{tabular}{lcccrr}
\hline \hline \multirow{2}{*}{ Method } & \multicolumn{2}{c}{ ImBz } & & \multicolumn{2}{c}{ PyBz } \\
\cline { 2 - 3 } \cline { 5 - 6 } dRPA(PBE) & -29.43 & -19.34 & & -27.20 & -17.09 \\
dRPA(PBE)+F12 & -21.79 & -20.78 & & -19.36 & -18.34 \\
dRPA(PBE)(F12) & -22.29 & -20.75 & & -19.87 & -18.30 \\
\hline \hline
\end{tabular}


Table 7: MP2-F12, SCS-MP2-F12, and SAPT-F12(MP2) binding energies ( $D_{0}$ in kJ/mol) obtained at SCS-CC2/aug-cc-pVTZ optimized geometries of the carbazole $\cdots X$ complexes $(\mathrm{X}=\mathrm{Ne}, \mathrm{Ar}, \mathrm{Kr}$, $\mathrm{Xe}, \mathrm{CO}, \mathrm{N}_{2}$, and $\mathrm{CH}_{4}$ ). Harmonic ZPVE from PBE-D3/def2-TZVP computations.

\begin{tabular}{|c|c|c|c|c|c|c|c|}
\hline Method & $\mathrm{Ne}$ & $\overline{\mathrm{Ar}}$ & $\mathrm{Kr}$ & $\overline{\mathrm{Xe}}$ & $\mathrm{CO}$ & $\mathrm{N}_{2}$ & $\overline{\mathrm{C} \mathrm{CH}_{4}}$ \\
\hline PBE-D3/def2-TZVP & 2.23 & 5.45 & 7.19 & 9.34 & 9.18 & 7.79 & 7.20 \\
\hline SCS-CC2/aug-cc-pVTZ & 1.00 & 5.42 & 7.39 & 9.42 & 7.19 & 6.78 & 6.55 \\
\hline MP2-F12 & 3.53 & 10.87 & 13.50 & 19.58 & 12.59 & 13.20 & 12.36 \\
\hline SCS-MP2-F12 & 2.54 & 7.43 & 9.05 & 12.62 & 8.25 & 8.60 & 7.88 \\
\hline SAPT-F12(MP2) & 1.91 & 6.38 & 8.47 & 11.04 & 7.42 & 6.90 & 7.86 \\
\hline Experiment $^{38,39,43}$ & 2.57 & 6.34 & 8.23 & 10.66 & 8.58 & 7.49 & 7.99 \\
\hline Exptl. error bar & \pm 0.004 & \pm 0.002 & \pm 0.05 & \pm 0.02 & \pm 0.38 & \pm 0.08 & \pm 0.17 \\
\hline
\end{tabular}


Table 8: Contributions (kJ/mol) to the PBE0AC-SAPT/aug-cc-pVTZ binding energies obtained at SCS-CC2/aug-cc-pVTZ optimized geometries of the carbazole $\cdots X$ complexes $(X=\mathrm{Ne}, \mathrm{Ar}, \mathrm{Kr}$, $\mathrm{Xe}, \mathrm{CO}, \mathrm{N}_{2}$, and $\mathrm{CH}_{4}$ ).

\begin{tabular}{|c|c|c|c|c|c|c|c|}
\hline Contribution & $\mathrm{Ne}$ & $\overline{\mathrm{Ar}}$ & $\mathrm{Kr}$ & $\mathrm{Xe}$ & $\mathrm{CO}$ & $\mathrm{N}_{2}$ & $\mathrm{CH}_{4}$ \\
\hline$E_{\mathrm{pol}}^{(1)}$ & -1.33 & -3.88 & -5.75 & -12.57 & -7.12 & -6.41 & -6.01 \\
\hline$E_{\mathrm{exch}}^{(1)}$ & 4.32 & 11.08 & 15.19 & 28.70 & 16.65 & 16.49 & 17.58 \\
\hline$E_{\text {ind }}^{(2)}$ & -1.63 & -5.14 & -9.24 & -22.39 & -6.21 & -5.66 & -5.29 \\
\hline$E_{\text {exch-ind }}^{(2)}$ & 1.70 & 4.95 & 8.87 & 19.23 & 5.68 & 5.31 & 4.99 \\
\hline$E_{\text {disp }}^{(2)}$ & -5.46 & -14.66 & -19.25 & -30.74 & -19.03 & -18.84 & -21.93 \\
\hline$E_{\text {exch-disp }}^{(2)}$ & 0.44 & 1.59 & 2.39 & 4.95 & 2.31 & 2.21 & 2.84 \\
\hline$\delta(\mathrm{HF})$ & -0.25 & -0.63 & -0.80 & 2.31 & -1.23 & -1.34 & -0.99 \\
\hline$\Delta \mathrm{F} 12$ & -0.17 & -0.32 & -0.43 & -1.26 & -0.40 & -0.38 & -0.29 \\
\hline $\operatorname{Total}\left(\Delta E_{2}\right)$ & -2.39 & -7.01 & -9.02 & -11.76 & -9.35 & -8.62 & -9.10 \\
\hline$\Delta E_{1}$ & 0.00 & 0.03 & 0.05 & 0.14 & 0.16 & 0.11 & 0.12 \\
\hline ZPVE/PBE-D3 & 0.48 & 0.60 & 0.49 & 0.58 & 1.77 & 1.61 & 1.12 \\
\hline$D_{0}$ SAPT-F12(MP2) & 1.91 & 6.38 & 8.47 & 11.04 & 7.42 & 6.90 & 7.86 \\
\hline$D_{0}$ Experiment $38,39,43$ & 2.57 & 6.34 & 8.23 & 10.66 & 8.58 & 7.49 & 7.99 \\
\hline Exptl. error bar & \pm 0.004 & \pm 0.002 & \pm 0.05 & \pm 0.02 & \pm 0.38 & \pm 0.08 & \pm 0.17 \\
\hline
\end{tabular}


Table 9: Selected ion and neutral dissociation energies $D_{0}$ (in $\mathrm{kJ} / \mathrm{mol}$ ) of dispersively bound aromatic M.S type-II complexes, measured by mass-analyzed threshold ionization (MATI), breakdown measurements (BM) two-color appearance potential (2C-AP), dispersed fluorescence (DIS) and velocity-map imaging (VMI).

\begin{tabular}{|c|c|c|c|c|c|c|}
\hline Complex & Method & Ion $D_{0}^{+}$ & Neutral $D_{0}$ & Calc. $D_{e}$ & Calc. $D_{0}$ & Refs. \\
\hline Benzene- $h_{6} \cdot \mathrm{CH}_{4}$ & MATI & $7.9 \pm 0.2$ & $4.5 \pm 0.2$ & 5.97 & 4.73 & 20 \\
\hline Benzene- $d_{6} \cdot \mathrm{CH}_{4}$ & MATI & $8.7 \pm 1.0$ & $5.5 \pm 1.0$ & 5.97 & 4.77 & 20 \\
\hline Benzene-ethene & 2C-AP & & $5.9 \pm 0.8$ & 9.06 & 7.2 & 21 \\
\hline Benzene-acetylene & 2C-AP & & $11.3 \pm 0.8$ & 11.5 & 10.0 & 21 \\
\hline Benzene $\cdot \mathrm{CH}_{2} \mathrm{Cl}_{2}$ & $2 \mathrm{C}-\mathrm{AP}$ & $7.6-8.1$ & $15.9 \pm 0.8$ & 18.7 & & 22 \\
\hline Benzene. $\mathrm{CHCl}_{3}$ & $2 \mathrm{C}-\mathrm{AP}$ & $7.6-9.7$ & $21.8 \pm 0.8$ & 22.8 & & 22 \\
\hline Benzene $\cdot \mathrm{N}_{2}$ & $\mathrm{BM}$ & & $3.8 \pm 0.3$ & & & 32 \\
\hline Benzene.cyclohexane & $\mathrm{BM}$ & & $7.7 \pm 2$ & & & 32 \\
\hline Benzene.cyclohexane & 2C-AP & & $11.3 \pm 1.3$ & & & 23 \\
\hline$p$-Difluorobenzene-benzene & $\mathrm{BM}$ & & $7.7 \pm 2$ & & & 25 \\
\hline$p$-Difluorobenzene $\cdot \mathrm{N}_{2}$ & DIS & & $<2.55$ & & & 303 \\
\hline Anisole $\cdot$ benzene & DIS & & $16.3-18.0$ & & & 255 \\
\hline Perylene $\cdot \mathrm{CO}_{2}$ & DIS & & $<14.8$ & & & 18 \\
\hline Perylene-ethylene & DIS & & $\approx 13.9$ & & & 18 \\
\hline Indole $\cdot \mathrm{CH}_{4}$ & DIS & & $<9.02$ & & & 299 \\
\hline Indole-benzene & MATI & & $21.8 \pm 0.18$ & & 22.2 & 29,33 \\
\hline Indole $\cdot$ benzene- $d_{6}$ & MATI & & $22.3 \pm 0.18$ & & & 29 \\
\hline
\end{tabular}


Table 10: Selected ion and neutral dissociation energies $D_{0}$ (in $\mathrm{kJ} / \mathrm{mol}$ ) of dispersively bound $\mathrm{M}_{2}$ and M·M' (type-III complexes) measured by Raman mass-analyzed threshold ionization (MATI), breakdown measurements (BM) two-color appearance potential (2C-AP), dispersed fluorescence (DIS) and velocity-map imaging (VMI).

\begin{tabular}{|c|c|c|c|c|c|c|}
\hline Complex & Method & Ion $D_{0}^{+}$ & Neutral $D_{0}$ & Calc. $D_{e}$ & Calc. $D_{0}$ & Refs. \\
\hline \multirow[t]{3}{*}{ (Benzene $)_{2}$} & Raman & & $11.87-12.4$ & 11.7 & 10.4 & $234,235,247$ \\
\hline & $\mathrm{BM}$ & $63.7 \pm 2$ & $6.75 \pm 1$ & & & 25 \\
\hline & 1C-AP & $64.03 \pm 4$ & $10.0 \pm 1.7$ & & & 237 \\
\hline$(\text { Naphthalene })_{2}$ & $\mathrm{BM}$ & 65.61 & 12.1 & 23.5 & & 304,305 \\
\hline \multirow[t]{2}{*}{$(\text { Anisole })_{2}$} & VMI & 73.5 & 47.0 & & & 36 \\
\hline & 2C-AP & $48.1 \pm 2.4$ & $21.5 \pm 1.2$ & $21.1 \pm 0.2$ & & 37 \\
\hline (Toluene $)_{2}$ & $\mathrm{BM}$ & & $14.5 \pm 1$ & 14.52 & & 25,250 \\
\hline Benzene toluene & $\mathrm{BM}$ & & $12.5 \pm 1$ & & & 25 \\
\hline$(p \text {-Difluorobenzene })_{2}$ & $\mathrm{BM}$ & & $8.7 \pm 2$ & & & 25 \\
\hline
\end{tabular}




\section{References}

(1) Meyer, E. A.; Castellano, R. K.; Diederich, F. Interactions with Aromatic Rings in Chemical and Biological Recognition. Angew. Chem. Int. Ed. 2003, 42, 1210-1250.

(2) Hobza, P.; Müller-Dethlefs, K. Non-Covalent Interactions: Theory and Experiment; The Royal Society of Chemistry: Cambridge, 2009.

(3) London, F. Zur Theorie und Systematik der Molekularkräfte. Z. Phys. 1930, 63, 245-279.

(4) Mons, M.; Dimicoli, I.; Piuzzi, F. Gas Phase Hydrogen-Bonded Complexes of Aromatic Molecules: Photoionization and Energetics. Int. Rev. Phys. Chem. 2002, 21, 101-135.

(5) Hobza, P.; Havlas, Z. Blue-Shifting Hydrogen Bonds. Chem. Rev. 2000, 100, 4253-4264.

(6) Braun, J. E.; Mehnert, T.; Neusser, H. J. Binding Energy of van der Waals- and Hydrogenbonded Clusters by Threshold Ionization Techniques. Int. J. Mass. Spec. 2000, 203, 1-18.

(7) Wormer, P. E. S.; van der Avoird, A. Intermolecular Potentials, Internal Motions, and Spectra of van der Waals and Hydrogen-Bonded Complexes. Chem. Rev. 2000, 100, 4109-4144.

(8) Topp, M. R. Dynamics and Structure of Aromatic Molecular van der Waals Complexes. Int. Rev. Phys. Chem. 1993, 12, 149-204.

(9) Haas, Y.; Kendler, S. Binding Energies of Aromatic 1:1 Hetero-Clusters in a Supersonic Jet. Isr. J. Chem. 1997, 37, 427-438.

(10) Haldar, S.; Gnanasekaran, R.; Hobza, P. A Comparison of Ab Initio Quantum-mechanical and Experimental $D_{0}$ Binding Energies of Eleven H-bonded and Eleven Dispersion-bound Complexes. Phys. Chem. Chem. Phys. 2015, 17, 26645-26652.

(11) Šponer, J.; Jurečka, P.; Hobza, P. Accurate Interaction Energies of Hydrogen-Bonded Nucleic Acid Base Pairs. J. Am. Chem. Soc. 2004, 126, 10142-10151. 
(12) Baer, T.; Hase, W. L. Unimolecular Reaction Dynamics; Oxford University Press (Oxford, New York), 1996.

(13) Meot-Ner, M.; Hamlet, P.; Hunter, E. P.; Field, F. H. Bonding Energies in Association Ions of Aromatic Compounds. Correlations with Ionization Energies. J. Am. Chem. Soc. 1978, $100,5466-5471$.

(14) Blazy, J. A.; DeKoven, B. M.; Russell, T. D.; Levy, D. H. The Binding Energy of IodineRare Gas van der Waals Molecules. J. Chem. Phys. 1980, 72, 2439-2444.

(15) Brumbaugh, D. V.; Kenny, J. E.; Levy, D. H. Vibrational Predissociation and Intramolecular Vibrational Relaxation in Electronically Excited s-Tetrazine-Argon van der Waals Complex. J. Chem. Phys. 1983, 78, 3415-3434.

(16) Bellm, S. M.; Gascooke, J. R.; Lawrance, W. D. The Dissociation Energy of van der Waals Complexes Determined by Velocity Map Imaging: Values for $S_{0}$ and $S_{1}$ p-DifluorobenzeneAr and $D_{0}$ (p-Difluorobenzene-Ar). Chem. Phy. Lett. 2000, 330, 103-109.

(17) Bellm, S. M.; Moulds, R. J.; Lawrance, W. D. The Binding Energies of p-DifluorobenzeneAr, Kr Measured by Velocity Map Imaging: Limitations of Dispersed Fluorescence in Determining Binding Energies. J. Chem. Phys. 2001, 115, 10709-10717.

(18) Wittmeyer, S. A.; Topp, M. R. Hot-Band Spectroscopy of Ground-State Levels of Perylene Following Predissociation of van der Waals Complexes. J. Phys. Chem. 1993, 97, 87188726.

(19) Tsuzuki, S.; Honda, K.; Uchimaru, T.; Mikami, M.; Fujii, A. Magnitude and Directionality of the Interaction of the Aliphatic CH/pi Interaction: Significant Difference from Hydrogen Bond. J. Phys. Chem. A 2006, 110, 10163-10168.

(20) Shibasaki, K.; Fujii, A.; Mikami, N.; Tsuzuki, S. Magnitude of the CH/pi Interaction in the 
Gas Phase: Experimental and Theoretical Determination of the Accurate Interaction Energy in Benzene-Methane. J. Phys. Chem. A 2006, 110, 4397-4404.

(21) Shibasaki, K.; Fujii, A.; Mikami, N.; Tsuzuki, S. Magnitude and Nature of Interactions in Benzene-X (X= Ethylene and Acetylene) in the Gas Phase: Significantly Different CH/pi Interaction of Acetylene As Compared with Those of Ethylene and Methane. J. Phys. Chem. A 2007, 111, 753-758.

(22) Tsuzuki, S.; Fujii, A. Nature and Physical Origins of CH/pi Interaction: Significant Difference from Conventional Hydrogen Bonds. Phys. Chem. Chem. Phys. 2008, 10, 2584-2594.

(23) Fujii, A.; Hayashi, H.; Park, J. W.; Kazama, T.; Mikami, N.; Tsuzuki, S. Experimental and Theoretical Determination of the Accurate $\mathrm{CH} /$ pi Interaction Energies in Benzene-Alkane Clusters: Correlation between Interaction Energy and Polarizability. Phys. Chem. Chem. Phys. 2011, 13, 14131-14141.

(24) Kiermeier, A.; Ernstberger, B.; Neusser, H. J.; Schlag, E. W. Multiphoton Mass Spectrometry of Clusters: Dissociation Kinetics of the Benzene Cluster Ions. J. Phys. Chem. 1988, 92, 3785-3789.

(25) Ernstberger, B.; Krause, H.; Kiermeier, A.; Neusser, H. J. Multiphoton Ionization and Dissociation of Mixed van der Waals Clusters in a Linear Reflectron Time-of-flight Mass Spectrometer. J. Chem. Phys 1990, 92, 5285-5296.

(26) Krause, H.; Neusser, H. J. Dissociation of State-Selected Complex-Ions Studied by Massselective Pulsed Field Threshold Ionization Spectroscopy. J. Chem. Phys. 1992, 97, 59235926.

(27) Krause, H.; Neusser, H. J. Dissociation-energy of Neutral and Ionic Benzene-Noble Gas Dimers by Pulsed-Field Threshold Ionization Spectroscopy. J. Chem. Phys. 1993, 99, 62786286. 
(28) Neusser, H. J.; Krause, H. Binding Energy and Structure of van der Waals Complexes of Benzene. Chem. Rev. 1994, 94, 1829-1843.

(29) Braun, J.; Neusser, H. J.; Hobza, P. NH. $\cdots \pi$ Interactions in Indole.benzene and Indole benzene- $h_{6}, d_{6}$ Radical Cation Complexes, Mass Analyzed Threshold Ionization Experiments and Correlated ab Initio Quantum Chemical Calculations. J. Phys. Chem. A 2003, $107,3918-3924$.

(30) Grebner, T. L.; Neusser, H. J. Vibrational Spectroscopy of Molecular and van der Waals Complex Cations by Mass Analyzed Pulsed Field Threshold Ionization Spectroscopy. Int. J. Mass Spectrom. Ion Processes 1996, 159, 137-152.

(31) Grebner, T. L.; Stumpf, R.; Neusser, H. J. Mass Analyzed Threshold Ionization of van der Waals Complexes: Binding Energies of Dibenzofuran·Ar and Dibenzo-p-dioxin·Ar. Int. J. Mass Spectrom. Ion Processes 1997, 167/168, 649-660.

(32) Ernstberger, B.; Krause, H.; Neusser, H. Metastable Decay and Binding Energies of van der Waals Cluster Ions. Z. Angew. Phys. D 1991, 20, 189-192.

(33) Braun, J. E.; Grebner, T. L.; Neusser, H. J. Van der Waals versus Hydrogen-bonding in Complexes of Indole with Argon, Water, and Benzene by Mass-analyzed Pulsed Field Threshold Ionization. J. Phys. Chem. A 1998, 102, 3273-3278.

(34) Sampson, R. K.; Lawrance, W. D. The Dissociation Energy of the Benzene-Argon van der Waals Complex Determined by Velocity Map Imaging. Aust. J. Chem. 2003, 56, 275-277.

(35) Bellm, S. M.; Moulds, R. J.; van Leeuwen, M. P.; Lawrance, W. D. A Velocity Map ion Imaging Study of Difluorobenzene-Water Complexes: Binding Energies and Recoil Distributions. J. Chem. Phys. 2008, 128, 114314.

(36) Mazzoni, F.; Pasquini, M.; Pietraperzia, G.; Becucci, M. Binding Energy Determination in 
a pi-stacked Aromatic Cluster: The Anisole Dimer. Phys. Chem. Chem. Phys. 2013, 15, $11268-11274$.

(37) Řezáč, J.; Nachtigallová, D.; Mazzoni, F.; Pasquini, M.; Pietraperzia, G.; Becucci, M.; Müller-Dethlefs, K.; Hobza, P. Binding Energies of the pi-Stacked Anisole Dimer: New Molecular Beam-Laser Spectroscopy Experiments and CCSD(T) Calculations. Chem. Eur. J. 2015, 21, 6740-6746.

(38) Droz, T.; Bürgi, T.; Leutwyler, S. Van der Waals Binding Energies and Intermolecular Vibrations of Carbazole-R (R=Ne, Ar, Kr, Xe). J. Chem. Phys. 1995, 103, 4035-4045.

(39) Bürgi, T.; Droz, T.; Leutwyler, S. Binding Energies of Carbazole-S van der Waals Complexes $\left(\mathrm{S}=\mathrm{N}_{2}, \mathrm{CO}\right.$, and $\left.\mathrm{CH}_{4}\right)$. J. Chem. Phys. 1995, 103, 7228-7239.

(40) Bürgi, T.; Droz, T.; Leutwyler, S. Accurate Hydrogen-Bonding Energies Between 1Naphthol and Water, Methanol and Ammonia. Chem. Phys. Lett. 1995, 246, 291-299.

(41) Wickleder, C.; Droz, T.; Bürgi, T.; Leutwyler, S. Accurate Intermolecular Binding Energies of 1-Naphthol to Benzene and Cyclohexane. Chem. Phys. Lett. 1997, 264, 257-264.

(42) Dai, H.-L.; Field, R. W. Molecular Dynamics and Spectroscopy by Stimulated Emission Pumping; World Scientific, Singapore, 1995.

(43) Bürgi, T.; Droz, T.; Leutwyler, S. Ground-State Binding Energy and Vibrations of the Carbazole-Ar van der Waals Complex by Pump/Dump-R2PI Measurements. Chem. Phys. Lett. 1994, 225, 351-358.

(44) Wickleder, C.; Henseler, D.; Leutwyler, S. Accurate Dissociation Energies of O-H..O hydrogen-bonded 1-Naphthol Solvent Complexes. J. Chem. Phys. 2002, 116, 1850-1857.

(45) Riley, K. E.; Pitoňák, M.; Jurečka, P.; Hobza, P. Stabilization and Structure Calculations for Noncovalent Interactions in Extended Molecular Systems Based on Wave Function and Density Functional Theories. Chem. Rev. 2010, 110, 5023-5063. 
(46) Hobza, P. Calculations on Noncovalent Interactions and Databases of Benchmark Interaction Energies. Acc. Chem. Res. 2012, 45, 663-672.

(47) Soydaş, E.; Bozkaya, U. Accurate Open-Shell Noncovalent Interaction Energies from the Orbital-Optimized Mø ller-Plesset Perturbation Theory: Achieving CCSD Quality at the MP2 Level by Orbital Optimization. J. Chem. Theory Comput. 2013, 9, 4679-4683.

(48) Bozkaya, U.; Sherrill, C. D. Orbital-optimized MP2.5 and its Analytic Gradients: Approaching CCSD(T) Quality for Noncovalent Interactions. J. Chem. Phys. 2014, 141, 204105.

(49) Pirani, F.; Maciel, G. S.; Cappelletti, D.; Aquilanti, V. Experimental Benchmarks and Phenomenology of Interatomic Forces: Open-Shell and Electronic Anisotropy Effects. Int. Rev. Phys. Chem. 2006, 25, 165-199.

(50) Hapka, M.; Żuchowski, P. S.; Szczȩśniak, M. M.; Chałasiński, G. Symmetry-adapted Perturbation Theory based on Unrestricted Kohn-Sham Orbitals for High-spin Open-shell van der Waals complexes. J. Chem. Phys. 2012, 137, 164104.

(51) Neiss, C.; Hättig, C.; Klopper, W. Extensions of R12 Corrections to CC2-R12 for Excited States. J. Chem. Phys. 2006, 125, 064111.

(52) Neiss, C.; Hättig, C. Frequency-Dependent Nonlinear Optical Properties with Explicitly Correlated Coupled-Cluster Response Theory Using the CCSD(R12) Model. J. Chem. Phys. 2007, $126,154101$.

(53) Köhn, A. A Modified Ansatz for Explicitly Correlated Coupled-cluster Wave Functions that is Suitable for Response Theory. J. Chem. Phys. 2009, 130, 104104.

(54) Hanauer, M.; Köhn, A. Response Properties with Explicitly Correlated Coupled-cluster Methods using a Slater-type Correlation Factor and Cusp Conditions. J. Chem. Phys. 2009, $131,124118$. 
(55) Pople, J. A.; Head-Gordon, M.; Raghavachari, K. Quadratic Configuration Interaction. A General Technique for Determining Electron Correlation Energies. J. Chem. Phys. 1987, $87,5968-5975$.

(56) Raghavachari, K.; Trucks, G. W.; Pople, J. A.; Head-Gordon, M. A Fifth-Order Perturbation Comparison of Electron Correlation Theories. Chem. Phys. Lett. 1989, 157, 479-483.

(57) Lee, T. J.; Rendell, A. P.; Taylor, P. R. Comparison of the Quadratic Configuration Interaction and Coupled-cluster Approaches to Electron Correlation Including the Effect of Triple Excitations. J. Phys. Chem. 1990, 94, 5463-5468.

(58) Bartlett, R. J. Coupled-cluster Theory and its Equation-of-motion Extensions. WIREs Comput. Mol. Sci. 2012, 2, 126-138.

(59) Cremer, D. From Configuration Interaction to Coupled-cluster Theory: The Quadratic Configuration Interaction Approach. WIREs Comput. Mol. Sci. 2013, 3, 482-503.

(60) Dunning, Jr., T. H. Gaussian Basis Sets for use in Correlated Molecular Calculations. I.The atoms Boron through Neon and Hydrogen. J. Chem. Phys. 1989, 90, 1007-1023.

(61) Kendall, R. A.; Dunning, Jr., T. H.; Harrison, R. J. Electron Affinities of the First-row Atoms Revisited. Systematic Basis Sets and Wave Functions. J. Chem. Phys. 1992, 96, 6796-6806.

(62) Raghavachari, K.; Saha, A. Accurate Composite and Fragment-Based Quantum Chemical Models for Large Molecules. Chem. Rev. 2015, 115, 5643-5677.

(63) Stoll, H. The Correlation Energy of Crystalline Silicon. Chem. Phys. Lett. 1992, 191, 548552.

(64) Stoll, H. Correlation Energy of Diamond. Phys. Rev. B 1992, 46, 6700-6704.

(65) Shukla, A.; Dolg, M.; Fulde, P.; Stoll, H. Wave-Function-Based Correlated Ab Initio Calculations on Crystalline Solids. Phys. Rev. B 1999, 60, 5211-5216. 
(66) Stoll, H.; Paulus, B.; Fulde, P. On the Accuracy of Correlation-Energy Expansions in Terms of Local Increments. J. Chem. Phys. 2005, 123, 144108.

(67) Li, S.; Shen, J.; Li, W.; Jiang, Y. An Efficient Implementation of the "Cluster-in-Molecule“ Approach for Local Electron Correlation Calculations. J. Chem. Phys. 2006, 125, 074109.

(68) Li, W.; Piecuch, P.; Gour, J. R.; Li, S. Local Correlation Calculations using Standard and Renormalized Coupled-cluster Approaches. J. Chem. Phys. 2009, 131, 114109.

(69) Li, W.; Chen, C.; Zhao, D.; Li, S. LSQC: Low Scaling Quantum Chemistry Program. Int. J. Quantum Chem. 2015, 115, 641-646.

(70) Kitaura, K.; Ikeo, E.; Asada, T.; Nakano, T.; Uebayasi, M. Fragment Molecular Orbital Method: An Approximate Computational Method for Large Molecules. Chem. Phys. Lett. 1999, 313, 701-706.

(71) Fedorov, D. G.; Kitaura, K. Coupled-Cluster Theory based upon the Fragment MolecularOrbital Method. J. Chem. Phys. 2005, 123, 134103.

(72) Hirata, S.; Valiev, M.; Dupuis, M.; Xantheas, S. S.; Sugiki, S.; Sekino, H. Fast Electron Correlation Methods for Molecular Clusters in the Ground and Excited States. Mol. Phys. 2005, 103, 2255-2265.

(73) Nakata, H.; Fedorov, D. G.; Nagata, T.; Kitaura, K.; Nakamura, S. Simulations of Chemical Reactions with the Frozen Domain Formulation of the Fragment Molecular Orbital Method. J. Chem. Theory Comput. 2015, 11, 3053-3064.

(74) Ziółkowski, M.; Jansík, B.; Kjærgaard, T.; Jørgensen, P. Linear Scaling Coupled Cluster Method with Correlation Energy Based Error Control. J. Chem. Phys. 2010, 133, 014107.

(75) Kristensen, K.; Ziółkowski, M.; Jansík, B.; Kjærgaard, T.; Jørgensen, P. A Locality Analysis of the Divide-Expand-Consolidate Coupled Cluster Amplitude Equations. J. Chem. Theory Comput. 2011, 7, 1677-1694. 
(76) Høyvik, I.-M.; Kristensen, K.; Jansík, B.; Jørgensen, P. The Divide-Expand-Consolidate Family of Coupled-cluster Methods: Numerical Illustrations using Second order MøllerPlesset Perturbation Theory. J. Chem. Phys. 2012, 136, 014105.

(77) Dahlke, E. E.; Truhlar, D. G. Electrostatically Embedded Many-Body Correlation Energy, with Applications to the Calculation of Accurate Second-Order Møller-Plesset Perturbation Theory Energies for Large Water Clusters. J. Chem. Theory Comput. 2007, 3, 1342-1348.

(78) Bygrave, P. J.; Allan, N. L.; Manby, F. R. The Embedded Many-body Expansion for Energetics of Molecular Crystals. J. Chem. Phys. 2012, 137, 164102.

(79) Friedrich, J.; Yu, H.; Leverentz, H. R.; Bai, P.; Siepmann, J. I.; Truhlar, D. G. Water 26mers Drawn from Bulk Simulations: Benchmark Binding Energies for Unprecedentedly Large Water Clusters and Assessment of the Electrostatically Embedded Three-Body and Pairwise Additive Approximations. J. Phys. Chem. Lett. 2014, 5, 666-670.

(80) Friedrich, J.; Hanrath, M.; Dolg, M. Fully Automated Implementation of the Incremental Scheme: Application to CCSD Energies for Hydrocarbons and Transition Metal Compounds. J. Chem. Phys. 2007, 126, 154110.

(81) Friedrich, J.; Tew, D. P.; Klopper, W.; Dolg, M. Automated Incremental Scheme for Explicitly Correlated Methods. J. Chem. Phys. 2010, 132, 164114.

(82) Friedrich, J.; Walczak, K. Incremental CCSD(T)(F12)|MP2-F12: A Method to Obtain Highly Accurate CCSD(T) Energies for Large Molecules. J. Chem. Theory Comput. 2013, 9, 408-417.

(83) Friedrich, J.; Hänchen, J. Incremental CCSD(T)(F12*)|MP2: A Black Box Method To Obtain Highly Accurate Reaction Energies. J. Chem. Theory Comput. 2013, 9, 5381-5394.

(84) Pulay, P. Localizability of Dynamic Electron Correlation. Chem. Phys. Lett. 1983, 100, 151154. 
(85) Hampel, C.; Werner, H.-J. Local Treatment of Electron Correlation in Coupled-cluster Theory. J. Chem. Phys. 1996, 104, 6286-6297.

(86) Schütz, M.; Werner, H.-J. Local Perturbative Triples Correction (T) with Linear Cost Scaling. Chem. Phys. Lett. 2000, 318, 370-378.

(87) Schütz, M. Low-Order Scaling Local Electron Correlation Methods. III. Linear Scaling Local Perturbative Triples Correction (T). J. Chem. Phys. 2000, 113, 9986-10001.

(88) Schütz, M.; Werner, H.-J. Low-Order Scaling Local Electron Correlation Methods. IV. Linear Scaling Local Coupled-Cluster (LCCSD). J. Chem. Phys. 2001, 114, 661-681.

(89) Werner, H.-J.; Schütz, M. An Efficient Local Coupled Cluster Method for Accurate Thermochemistryof Large Systems. J. Chem. Phys. 2011, 135, 144116.

(90) Masur, O.; Usvyat, D.; Schütz, M. Efficient and Accurate Treatment of Weak Pairs in Local CCSD(T) Calculations. J. Chem. Phys. 2013, 139, 164116.

(91) Schütz, M.; Masur, O.; Usvyat, D. Efficient and Accurate Treatment of Weak Pairs in Local $\operatorname{CCSD}(\mathrm{T})$ Calculations. II. Beyond the Ring Approximation. J. Chem. Phys. 2014, 140, 244107.

(92) Schwilk, M.; Usvyat, D.; Werner, H.-J. Communication: Improved Pair Approximations in Local Coupled-Cluster Methods. J. Chem. Phys. 2015, 142, 121102.

(93) Neese, F.; Wennmohs, F.; Hansen, A. Efficient and Accurate Local Approximations to Coupled-Electron Pair Approaches: An Attempt to Revive the Pair Natural Orbital Method. J. Chem. Phys. 2009, 130, 114108.

(94) Neese, F.; Hansen, A.; Liakos, D. G. Efficient and Accurate Approximations to the Local Coupled Cluster Singles Doubles Method Using a Truncated Pair Natural Orbital Basis. J. Chem. Phys. 2009, 131, 064103. 
(95) Liakos, D. G.; Hansen, A.; Neese, F. Weak Molecular Interactions Studied with Parallel Implementations of the Local Pair Natural Orbital Coupled Pair and Coupled Cluster Methods. J. Chem. Theory Comput. 2011, 7, 76-87.

(96) Riplinger, C.; Neese, F. An Efficient and Near Linear Scaling Pair Natural Orbital Based Local Coupled Cluster Method. J. Chem. Phys. 2013, 138, 034106.

(97) Riplinger, C.; Sandhoefer, B.; Hansen, A.; Neese, F. Natural Triple Excitations in Local Coupled Cluster Calculations with Pair Natural Orbitals. J. Chem. Phys. 2013, 139, 134101.

(98) Rolik, Z.; Szegedy, L.; Ladjánszki, I.; Ladóczki, B.; Kállay, M. An Efficient Linear-Scaling CCSD(T) Method Based on Local Natural Orbitals. J. Chem. Phys. 2013, 139, 094105.

(99) Řezáč, J.; Riley, K. E.; Hobza, P. S66: A Well-balanced Database of Benchmark Interaction Energies Relevant to Biomolecular Structures. J. Chem. Theory Comput. 2011, 7, 24272438.

(100) Řezáč, J.; Riley, K. E.; Hobza, P. Erratum to' S66: A Well-balanced Database of Benchmark Interaction Energies Relevant to Biomolecular Structures '. J. Chem. Theory Comput. 2014, $10,1359-1360$.

(101) Schmitz, G.; Hättig, C.; Tew, D. P. Explicitly Correlated PNO-MP2 and PNO-CCSD and Their Application to the S66 Set and Large Molecular Systems. Phys. Chem. Chem. Phys. 2014, 16, 22167-22178.

(102) Liakos, D. G.; Sparta, M.; Kesharwani, M. K.; Martin, J. M. L.; Neese, F. Exploring the Accuracy Limits of Local Pair Natural Orbital Coupled-Cluster Theory. J. Chem. Theory Comput. 2015, 11, 1525-1539.

(103) Liakos, D. G.; Neese, F. Domain Based Pair Natural Orbital Coupled Cluster Studies on Linear and Folded Alkane Chains. J. Chem. Theory Comput. 2015, 11, 2137-2143. 
(104) Adler, T. B.; Werner, H.-J. An Explicitly Correlated Local Coupled Cluster Method for Calculations of Large Molecules Close to the Basis Set Limit. J. Chem. Phys. 2011, 135, 144117.

(105) Tew, D. P.; Helmich, B.; Hättig, C. Local Explicitly Correlated Second-Order Møller-Plesset Perturbation Theory with Pair Matural Orbitals. J. Chem. Phys. 2011, 135, 074107.

(106) Hättig, C.; Tew, D. P.; Helmich, B. Local Explicitly Correlated Second- and Third-order Møller-Plesset Perturbation Theory with Pair Natural Orbitals. J. Chem. Phys. 2012, 136, 204105.

(107) Krause, C.; Werner, H.-J. Comparison of Explicitly Correlated Local Coupled-cluster Methods with various Choices of Virtual Orbitals. Phys. Chem. Chem. Phys. 2012, 14, 75917604.

(108) Pavošević, F.; Neese, F.; Valeev, E. F. Geminal-Spanning Orbitals make Explicitly Correlated Reduced-Scaling Coupled-Cluster Methods Robust, Yet Simple. J. Chem. Phys. 2014, $141,054106$.

(109) Harding, M. E.; Klopper, W. Benchmarking the Lithium-Thiophene Complex. ChemPhysChem 2013, 14, 708-715.

(110) Ahnen, S.; Hehn, A.-S.; Vogiatzis, K. D.; Trachsel, M. A.; Leutwyler, S.; Klopper, W. Accurate Computations of the Structures and Binding Energies of the Imidazolebenzene and Pyrrolebenzene Complexes. Chem. Phys. 2014, 441, 17-22.

(111) Fliegl, H.; Klopper, W.; Hättig, C. Coupled-cluster Theory with Simplified Linear-R12 Corrections: The CCSD(R12) Model. J. Chem. Phys. 2005, 122, 084107.

(112) Ten-no, S.; Noga, J. Explicitly Correlated Electronic Structure Theory from R12/F12 Ansätze. WIREs Comput. Mol. Sci. 2012, 2, 114-125. 
(113) Kong, L.; Bischoff, F. A.; Valeev, E. F. Explicitly Correlated R12/F12 Methods for Electronic Structure. Chem. Rev. 2012, 112, 75-107.

(114) Hättig, C.; Klopper, W.; Köhn, A.; Tew, D. P. Explicitly Correlated Electrons in Molecules. Chem. Rev. 2012, 112, 4-74.

(115) Knizia, G.; Adler, T. B.; Werner, H.-J. Simplified CCSD(T)-F12 Methods: Theory and Benchmarks. J. Chem. Phys. 2009, 130, 054104.

(116) van Duijneveldt, F. B.; van Duijneveldt-van de Rijdt, J. G. C. M.; van Lenthe, J. H. State of the Art in Counterpoise Theory. Chem. Rev. 1994, 94, 1873-1885.

(117) Burns, L. A.; Marshall, M. S.; Sherrill, C. D. Comparing Counterpoise-Corrected, Uncorrected, and Averaged Binding Energies for Benchmarking Noncovalent Interactions. J. Chem. Theory Comput. 2014, 10, 49-57.

(118) Leist, R.; Frey, J.; Ottiger, P.; Frey, H.-M.; Leutwyler, S.; Bachorz, R.; Klopper, W. Nucleobase-Fluorobenzene Interactions: Hydrogen Bonding Wins over $\pi$ Stacking. Angew. Chem. Int. Ed. 2007, 46, 7449-7452.

(119) Pitoňák, M.; Neogrády, P.; Černý, J.; Grimme, S.; Hobza, P. Scaled MP3 NonCovalent Interaction Energies Agree Closely with Accurate CCSD(T) Benchmark Data. ChemPhysChem 2009, 10, 282-289.

(120) Vogiatzis, K. D.; Klopper, W. Accurate Non-Covalent Interactions with Basis-Set Corrections from Interference-Corrected Perturbation Theory: Comparison with the S22B Database. Mol. Phys. 2013, 111, 2299-2305.

(121) Jurečka, P.; Šponer, J.; Černý, J.; Hobza, P. Benchmark Database of Accurate (MP2 and CCSD(T) Complete Basis Set Limit) Interaction Energies of Small Model Complexes, DNA Base Pairs, and Amino Acid Pairs. Phys. Chem. Chem. Phys. 2006, 8, 1985-1993. 
(122) Marshall, M. S.; Burns, L. A.; Sherrill, C. D. Basis Set Convergence of the Coupled-cluster Correction, MP2CCSD(T): Best Practices for Benchmarking Non-covalent Interactions and the Attendant Revision of the S22, NBC10, HBC6, and HSG Databases. J. Chem. Phys. 2011, 135, 194102.

(123) Vogiatzis, K. D.; Klopper, W.; Friedrich, J. Non-Covalent Interactions of $\mathrm{CO}_{2}$ with Functional Groups of Metal-Organic Frameworks from a CCSD(T) Scheme Applicable to Large Systems. J. Chem. Theory Comput. 2015, 11, 1574-1584.

(124) Halkier, A.; Helgaker, T.; Jørgensen, P.; Klopper, W.; Koch, H.; Olsen, J.; Wilson, A. K. Basis-set Convergence in Correlated Calculations on $\mathrm{Ne}, \mathrm{N}_{2}$, and $\mathrm{H}_{2} \mathrm{O}$. Chem. Phys. Lett. 1998, 286, 243-252.

(125) Halkier, A.; Helgaker, T.; Jørgensen, P.; Klopper, W.; Olsen, J. Chem. Phys. Lett. 1999, 302, 437-446.

(126) Sinnokrot, M. O.; Valeev, E. F.; Sherrill, C. D. Estimates of the Ab Initio Limit for $\pi-\pi$ Interactions: The Benzene Dimer. J. Am. Chem. Soc. 2002, 124, 10887-10893.

(127) Schwenke, D. W. The Extrapolation of One-Electron Basis Sets in Electronic Structure Calculations: How it Should Work and How it Can Be Made to Work. J. Chem. Phys. 2005, $122,014107$.

(128) Sedlak, R.; Janowski, T.; Pitoňák, M.; Řezáč, J.; Pulay, P.; Hobza, P. Accuracy of Quantum Chemical Methods for Large Noncovalent Complexes. J. Chem. Theory Comput. 2013, 9 , $3364-3374$.

(129) Papajak, E.; Zheng, J.; Xu, X.; Leverentz, H. R.; Truhlar, D. G. Perspectives on Basis Sets Beautiful: Seasonal Plantings of Diffuse Basis Functions. J. Chem. Theory Comput. 2011, 7, 3027-3034. 
(130) Grimme, S.; Goerigk, L.; Fink, R. F. Spin-component-scaled Electron Correlation Methods. WIREs Comput. Mol. Sci. 2012, 2, 886-906.

(131) Pfaffen, C.; Frey, H.-M.; Ottiger, P.; Leutwyler, S.; Bachorz, R. A.; Klopper, W. LargeAmplitude Vibrations of an NH- $\pi$ Hydrogen Bonded cis-Amide-Benzene Complex. Phys. Chem. Chem. Phys. 2010, 12, 8208-8218.

(132) Ottiger, P.; Pfaffen, C.; Leist, R.; Leutwyler, S.; Bachorz, R. A.; Klopper, W. Strong NH $\cdots \pi$ Hydrogen Bonding in Amide-Benzene Interactions. J. Phys. Chem. B 2009, 113, 29372943.

(133) Bachorz, R. A.; Bischoff, F. A.; Hofener, S.; Klopper, W.; Ottiger, P.; Leist, R.; Frey, J. A.; Leutwyler, S. Scope and Limitations of the SCS-MP2 Method for Stacking and Hydrogen Bonding Interactions. Phys. Chem. Chem. Phys. 2008, 10, 2758-2766.

(134) Goerigk, L.; Grimme, S. Assessment of TD-DFT Methods and of Various Spin-scaled CIS(D) and CC2 Versions for the Treatment of Low-lying Valence Excitations of Large Organic Dyes. J. Chem. Phys. 2010, 132, 184103.

(135) Grimme, S.; Izgorodina, E. I. Calculation of 0âĂŞ0 Excitation Energies of Organic Molecules by CIS(D) Quantum Chemical Methods. Chem. Phys. 2004, 305, 223-230.

(136) Hättig, C. In Response Theory and Molecular Properties (A Tribute to Jan Linderberg and Poul Jørgensen); Jensen, H. J. A., Ed.; Adv. Quantum Chem.; Academic Press, 2005; Vol. 50; pp 37-60.

(137) Rhee, Y. M.; Head-Gordon, M. Scaled Second-Order Perturbation Corrections to Configuration Interaction Singles: Efficient and Reliable Excitation Energy Methods. J. Phys. Chem. A 2007, 111, 5314-5326.

(138) Hellweg, A.; Grün, S. A.; Hättig, C. Benchmarking the Performance of Spin-component- 
scaled CC2 in Ground and Electronically Excited States. Phys. Chem. Chem. Phys. 2008, $10,4119-4127$.

(139) Misquitta, A. J.; Szalewicz, K. Intermolecular Forces from Asymptotically Corrected Density Functional description of Monomers. Chem. Phys. Lett. 2002, 357, 301-306.

(140) Misquitta, A. J.; Jeziorski, B.; Szalewicz, K. Dispersion Energy from Density-Functional Theory Description of Monomers. Phys. Rev. Lett. 2003, 91, 033201.

(141) Misquitta, A. J.; Szalewicz, K. Symmetry-Adapted Perturbation-Theory Calculations of Intermolecular Forces employing Density-Functional Description of Monomers. J. Chem. Phys. 2005, 122, 214109.

(142) Jeziorski, B.; Moszynski, R.; Szalewicz, K. Perturbation Theory Approach to Intermolecular Potential Energy Surfaces of van der Waals Complexes. Chem. Rev. 1994, 94, 1887-1930.

(143) Szalewicz, K. Symmetry-Adapted Perturbation Theory of Intermolecular Forces. WIREs Comput. Mol. Sci. 2012, 2, 254-272.

(144) Jansen, G. Symmetry-adapted Perturbation Theory based on Density Functional Theory for Noncovalent Interactions. WIREs Comput. Mol. Sci. 2014, 4, 127-144.

(145) Lao, K. U.; Herbert, J. M. Accurate and Efficient Quantum Chemistry Calculations for Noncovalent Interactions in Many-Body Systems: The XSAPT Family of Methods. J. Phys. Chem. A 2015, 119, 235-252.

(146) Sherrill, C. D. Energy Component Analysis of $\pi$ Interactions. Acc. Chem. Res. 2013, 46, $1020-1028$.

(147) Misquitta, A. J.; Podeszwa, R.; Jeziorski, B.; Szalewicz, K. Intermolecular Potentials based on Symmetry-adapted Perturbation Theory with Dispersion Energies from Time-dependent Density-functional calculations. J. Chem. Phys. 2005, 123, 214103. 
(148) Heßelmann, A.; Janssen, G. Chem. Phys. Lett. 2002, 362, 319-325.

(149) Heßelmann, A.; Janssen, G.; Schütz, M. Density-functional Theory-symmetry-adapted Intermolecular Perturbation Theory with Density Fitting: A new Efficient Method to Study Intermolecular Interaction Energies. J. Chem. Phys. 2005, 122, 014103.

(150) Perdew, J. P.; Ernzerhof, M.; Burke, K. Rationale for Mixing Exact Exchange with Density Functional Approximations. J. Chem. Phys. 1996, 105, 9982-9985.

(151) Grüning, M.; Gritsenko, O. V.; van Gisbergen, S. J. A.; Baerends, E. J. Shape Corrections to Exchange-correlation Potentials by Gradient-regulated Seamless Connection of Model Potentials for Inner and Outer Region. J. Chem. Phys. 2001, 114, 652-660.

(152) Grimme, S. Density Functional Theory with London Dispersion Corrections. WIREs Comput. Mol. Sci. 2011, 1, 211-228.

(153) Ehrlich, S.; Moellmann, J.; Grimme, S. Dispersion-Corrected Density Functional Theory for Aromatic Interactions in Complex Systems. Acc. Chem. Res. 2013, 46, 916-926.

(154) Tkatchenko, A.; Scheffler, M. Accurate Molecular van der Waals Interactions from GroundState Electron Density and Free-Atom Reference Data. Phys. Rev. Lett. 2009, 102, 073005.

(155) Ferri, N.; DiStasio, Jr., R. A.; Ambrosetti, A.; Car, R.; Tkatchenko, A. Electronic Properties of Molecules and Surfaces with a Self-Consistent Interatomic van der Waals Density Functional. Phys. Rev. Lett. 2015, 114, 176802.

(156) Becke, A. D.; Johnson, E. R. Exchange-hole Dipole Moment and the Dispersion Interaction. J. Chem. Phys. 2005, 122, 154104.

(157) Becke, A. D.; Johnson, E. R. A Density-functional Model of the Dispersion Interaction. $J$. Chem. Phys. 2005, 123, 154101.

(158) Becke, A. D.; Johnson, E. R. Exchange-hole Dipole Moment and the Dispersion Interaction Revisited. J. Chem. Phys. 2007, 127, 154108. 
(159) Toulouse, J.; Zhu, W.; Ángyán, J. G.; Savin, A. Range-Separated Density-Functional Theory with the Random-Phase Approximation: Detailed Formalism and Illustrative Applications. Phys. Rev. A 2010, 82, 032502.

(160) Zhu, W.; Toulouse, J.; Savin, A.; Ángyán, J. G. Range-Separated Density-Functional Theory with Random Phase Approximation Applied to Noncovalent Intermolecular Interactions. J. Chem. Phys. 2010, 132, 244108.

(161) Eshuis, H.; Bates, J. E.; Furche, F. Electron Correlation Methods based on the Randomphase Approximation. Theor. Chem. Acc. 2012, 131, 1084.

(162) Eshuis, H.; Furche, F. Basis Set Convergence of Molecular Correlation Energy Differences within the Random-phase Approximation. J. Chem. Phys. 2012, 136, 084105.

(163) Heßelmann, A.; Görling, A. On the Short-Range Behavior of Correlated Pair Functions from the Adiabatic-Connection Fluctuation-Dissipation Theorem of Density-Functional Theory. J. Chem. Theory Comput. 2013, 9, 4382-4395.

(164) Bleiziffer, P.; Heßelmann, A.; Görling, A. Efficient Self-consistent Treatment of Electron Correlation within the Random Phase Approximation. J. Chem. Phys. 2013, 139, 084113.

(165) Burow, A. M.; Bates, J. E.; Furche, F.; Eshuis, H. Analytical First-Order Molecular Properties and Forces within the Adiabatic Connection Random Phase Approximation. J. Chem. Theory Comput. 2014, 10, 180-194.

(166) Krause, K.; Klopper, W. Communication: Two-component Ring-coupled-cluster Computation of the Correlation Energy in the Random-phase Approximation. J. Chem. Phys. 2013, $139,191102$.

(167) Kühn, M. Correlation Energies from the Two-Component Random Phase Approximation. J. Chem. Theory Comput. 2014, 10, 623-633. 
(168) Kállay, M. Linear-scaling Implementation of the Direct Random-phase Approximation. $J$. Chem. Phys. 2015, 142, 204105.

(169) Hehn, A.-S.; Klopper, W. Communication: Explicitly-correlated Second-order Correction to the Correlation Energy in the Random-phase Approximation. J. Chem. Phys. 2013, 138, 181104.

(170) Hehn, A.-S.; Tew, D. P.; Klopper, W. Explicitly Correlated Ring-coupled-cluster-doubles Theory. J. Chem. Phys. 2015, 142, 194106.

(171) Bates, J. E.; Furche, F. Communication: Random Phase Approximation Renormalized Many-body Perturbation Theory. J. Chem. Phys. 2013, 139, 171103.

(172) Grüneis, A.; Marsman, M.; Harl, J.; Schimka, L.; Kresse, G. Making the Random-phase Approximation to Electronic Correlation Accurate. J. Chem. Phys. 2009, 131, 154115.

(173) Ren, X.; Rinke, P.; Scuseria, G. E.; Scheffler, M. Renormalized Second-Order Perturbation Theory for the Electron Correlation energy: Concept, Implementation, and Benchmarks. Phys. Rev. B 2013, 88, 035120.

(174) Franck, O.; Mussard, B.; Luppi, E.; Toulouse, J. Basis Convergence of Range-separated Density-functional Theory. J. Chem. Phys. 2015, 142, 074107.

(175) Korth, M.; Pitoňák, M.; Řezáč, J.; Hobza, P. A Transferable H-Bonding Correction for Semiempirical Quantum-Chemical Methods. J. Chem. Theory Comput. 2010, 6, 344-352.

(176) Elstner, M.; Hobza, P.; Frauenheim, T.; Suhai, S.; Kaxiras, E. Hydrogen Bonding and Stacking Interactions of Nucleic Acid Base Pairs: A Density-functional Theory based Treatment. J. Chem. Phys. 2001, 114, 5149-5155.

(177) Brandenburg, J. G.; Grimme, S. Accurate Modeling of Organic Molecular Crystals by Dispersion-Corrected Density Functional Tight Binding (DFTB). J. Phys. Chem. Lett. 2014, $5,1785-1789$. 
(178) Dubecký, M.; Jurečka, P.; Derian, R.; Hobza, P.; Otyepka, M.; Mitas, L. Quantum Monte Carlo Methods Describe Noncovalent Interactions with Subchemical Accuracy. J. Chem. Theory Comput. 2013, 9, 4287-4292.

(179) Dubecký, M.; Derian, R.; Jurečka, P.; Mitas, L.; Hobza, P.; Otyepka, M. Quantum Monte Carlo for Noncovalent Interactions: An Efficient Protocol Attaining Benchmark Accuracy. Phys. Chem. Chem. Phys. 2014, 16, 20915-20923.

(180) Řezáč, J.; Dubecký, M.; Jurečka, P.; Hobza, P. Extensions and Applications of the A24 Data Set of Accurate Interaction Energies. Phys. Chem. Chem. Phys. 2015, 17, 19268-19277.

(181) Gosling, M. P.; Cockett, M. C. R. Hydrogen-bonding in the Pyrimidine- $\mathrm{NH}_{3}$ van der Waals Complex: Experiment and Theory. Phys. Chem. Chem. Phys. 2014, 16, 14195-14205.

(182) Cui, G.; Guan, P.-J.; Fang, W.-H. Photoinduced Proton Transfer and Isomerization in a Hydrogen-Bonded Aromatic Azo Compound: A CASPT2//CASSCF Study. J. Phys. Chem. A 2014, 118, 4732-4739.

(183) Huenerbein, R.; Grimme, S. Time-dependent Density Functional Study of Excimers and Exciplexes of Organic Molecules. Chem. Phys. 2008, 343, 362-371.

(184) Furche, F.; Ahlrichs, R.; Hättig, C.; Klopper, W.; Sierka, M.; Weigend, F. Turbomole. WIREs Comput. Mol. Sci. 2014, 4, 91-100.

(185) TURBOMOLE, Version 7.0, a Development of Universität Karlsruhe (TH) and Forschungszentrum Karlsruhe GmbH, 1989-2007, TURBOMOLE GmbH, since 2007. Available from http: / / www.turbomole.com.

(186) Weigend, F.; Furche, F.; Ahlrichs, R. Gaussian Basis Sets of Quadruple Zeta Valence Quality for Atoms H-Kr. J. Chem. Phys. 2003, 119, 12753-12762.

(187) Christiansen, O.; Stanton, J. F.; Gauss, J. A Coupled-cluster Study of the ${ }^{1} A_{1 g}$ and ${ }^{1} B_{2 u}$ States of Benzene. J. Chem. Phys. 1998, 108, 3987-4001. 
(188) Kolmann, S. J.; D’Arcy, J. H.; Jordan, M. J. T. Quantum Effects and Anharmonicity in the H2-Li+-Benzene Complex: A Model for Hydrogen Storage Materials. J. Chem. Phys. 2013, $139,234305$.

(189) Mandziuk, M.; Bačić, Z. Van der Waals Vibrational States of Atom-Large Molecule Complexes by a 3D Discrete Variable Representation Method: Naphthalene-Ar. J. Chem. Phys. 1993, 98, 7165-7178.

(190) Mandziuk, M.; Bačić, Z.; Droz, T.; Leutwyler, S. Intermolecular Vibrations of the 2,3-Dimethylnaphthalene-Ar van der Waals complex: Experiment and Quantum Threedimensional Calculations. J. Chem. Phys. 1994, 100, 52-62.

(191) Droz, T.; Leutwyler, S.; Mandziuk, M.; Bačić, Z. Intermolecular Vibrations of o-Xylene-Ar in the $S_{0}$ and $S_{1}$ States: Experiment and Quantum Three Dimensional Calculations. J. Chem. Phys. 1994, 101, 6412-6423.

(192) Droz, T.; Leutwyler, S.; Mandziuk, M.; Bačić, Z. Van der Waals Vibrations and Isomers of 2,3-Dimethylnaphthalene-Ne: Experiment and Quantum Three-Dimensional Calculations. J. Chem. Phys. 1995, 103, 4855-4868.

(193) Bach, A.; Leutwyler, S.; Sabo, D.; Bačić, Z. Very Large Amplitude Intermolecular Vibrations and Wave Function Delocalization in 2,3-Dimethylnaphthalene-He van der Waals Complex. J. Chem. Phys. 1997, 107, 8781-8793.

(194) Cybulski, H.; Fernández, B.; Henriksen, C.; Felker, P. M. Ab Initio Ground State Phenylacetylene-Argon Intermolecular Potential Energy Surface and Rovibrational Spectrum. J. Chem. Phys. 2012, 137, 074305.

(195) Cagide Fajín, J. L.; Fernández, B.; Felker, P. M. p-Difluorobenzene-Argon Ground State Intermolecular Potential Energy Surface. J. Phys. Chem. A 2005, 109, 11602-11608. 
(196) Cagide Fajín, J. L.; Fernández, B.; Felker, P. M. The p-Difluorobenzene-Argon $S_{1}$ Excited State Intermolecular Potential Energy Surface. J. Phys. Chem. A 2006, 110, 13259-13263.

(197) Bettens, R. P. A.; Collins, M. A. Learning to Interpolate Molecular Potential Energy Surfaces with Confidence: A Bayesian Approach. J. Chem. Phys. 1999, 111, 816-826.

(198) Kalescky, R.; Kraka, E.; Cremer, D. Accurate Determination of the Binding Energy of the Formic Acid Dimer: The Importance of Geometry Relaxation. J. Chem. Phys. 2014, 140, 084315.

(199) Panek, P. T.; Jacob, C. R. Efficient Calculation of Anharmonic Vibrational Spectra of Large Molecules with Localized Modes. ChemPhysChem 2014, 15, 3365-3377.

(200) Pfeiffer, F.; Rauhut, G.; Feller, D.; Peterson, K. A. Anharmonic Zero Point Vibrational Energies: Tipping the Scales in Accurate Thermochemistry Calculations? J. Chem. Phys. 2013, 138, 044311.

(201) Weitzel, K.-M.; Mähnert, J. The Binding Energies of Small $\mathrm{Ar}_{n},(\mathrm{CO})_{n}$ and $\left(\mathrm{N}_{2}\right)_{n}$ Cluster Ions. Int. J. Mass Spectrom. 2002, 214, 175-212.

(202) Colbourn, E. A.; Douglas, A. E. The Spectrum and Ground State Potential Curve of Ar $2 . J$. Chem. Phys. 1976, 65, 1741-1745.

(203) Herman, P. R.; LaRocque, P. E.; Stoicheff, B. P. Vacuum Ultraviolet Laser Spectroscopy. V. Rovibronic Spectra of $\mathrm{Ar}_{2}$ and Constants of the Ground and Excited States. J. Chem. Phys. 1988, $89,4535-4549$.

(204) Signorell, R.; Merkt, F. The First Electronic States of $\mathrm{Ar}_{2}^{+}$Studied by High Resolution Photoelectron Spectroscopy. J. Chem. Phys. 1998, 109, 9762-9771.

(205) Signorell, R.; Wüst, A.; Merkt, F. The First Adiabatic Ionization Potential of Ar $2 . J$. Chem. Phys. 1997, 107, 10819-10822. 
(206) Onuma, T.; Yoshii, H.; Ishijima, H.; Itou, Y.; Hayaishi, T.; Morioka, Y. Pulsed-Field Ionization Zero-Kinetic-Energy Photoelectron Spectra of $\mathrm{Ar}_{2}$. J. Mol. Spectrosc. 1999, 198, 209-217.

(207) Signorell, R.; Hollenstein, U.; Merkt, F. High-Resolution Photoelectron Spectroscopic Study of the First Electronic States of $\mathrm{Kr}_{2}^{+}$. J. Chem. Phys. 2001, 114, 9840-9851.

(208) Rupper, P.; Zehnder, O.; Merkt, F. Potential Energy Curves of Diatomic Molecular Ions from High-Resolution Photoelectron Spectra. II. The First Six Electronic States of $\mathrm{Xe}_{2}^{+} . J$. Chem. Phys. 2004, 121, 8279-8290.

(209) Zehnder, O.; Merkt, F. The Low-Lying Electronic States of $\mathrm{KrXe}^{+}$and their Potential Energy Functions. Mol. Phys. 2008, 106, 1215-1226.

(210) Zehnder, O.; Merkt, F. The Low-Lying Electronic States of $\mathrm{ArXe}^{+}$and their Potential Energy Functions. J. Chem. Phys. 2008, 128, 014306.

(211) Rocher-Casterline, B. E.; Ch’'ng, L. C.; Mollner, A. K.; Reisler, H. Communication: Determination of the Bond Dissociation Energy $\left(D_{0}\right)$ of the Water Dimer, $\left(\mathrm{H}_{2} \mathrm{O}\right)_{2}$, by Velocity Map Imaging. J. Chem. Phys 2011, 134, 211101.

(212) Ch’ng, L. C.; Samanta, A. K.; Czakó, G.; Bowman, J. M.; Reisler, H. Experimental and Theoretical Investigations of Energy Transfer and Hydrogen-Bond Breaking in the Water Dimer. J. Am. Chem. Soc. 2012, 134, 15430-15435.

(213) Shank, A.; Wang, Y.; Kaledin, A.; Braams, B. J.; Bowman, J. M. Accurate ab initio and "Hybrid" Potential Energy Surfaces, Intramolecular Vibrational Energies, and Classical IR Spectrum of the Water Dimer. J. Chem. Phys. 2009, 130, 144314.

(214) Czakó, G.; Wang, Y.; Bowman, J. M. Communication: Quasiclassical Trajectory Calculations of Correlated Product-state Distributions for the Dissociation of $\left(\mathrm{H}_{2} \mathrm{O}\right)_{2}$ and $\left(\mathrm{D}_{2} \mathrm{O}\right)_{2}$. J. Chem. Phys 2011, 135, 151102. 
(215) Klopper, W.; van Duijneveldt-van de Rijdt, J. G. C. M.; van Duijneveldt, F. B. Computational Determination of Equilibrium Geometry and Dissociation Energy of the Water Dimer. Phys. Chem. Chem. Phys. 2000, 2, 2227-2234.

(216) Samanta, A. K.; Czakó, G.; Wang, Y.; Mancini, J. S.; Bowman, J. M.; Reisler, H. Experimental and Theoretical Investigations of Energy Transfer and Hydrogen-Bond Breaking in Small Water and HCl Clusters. Acc. Chem. Res. 2014, 47, 2700-2709.

(217) Case, A. S.; Heid, C. G.; Kable, S. H.; Crim, F. F. Dissociation Energy and Vibrational Predissociation Dynamics of the Ammonia Dimer. J. Chem. Phys. 2011, 135, 084312.

(218) Oudejans, L.; Miller, R. E. Photofragment Translational Spectroscopy of Weakly Bound Complexes: Probing the Interfragment Correlated Final State Distributions. Annu. Rev. Phys. Chem. 2001, 52, 607-637.

(219) Neusser, H. J.; Sussmann, R.; Smith, A. M.; Riedle, E.; Weber, T. High-Resolution UV Spectroscopy of Molecular Complexes. Ber. Bunsenges. Phys. Chem. 1992, 96, 1252-1259.

(220) Chewter, L. A.; Müller-Dethlefs, K.; Schlag, E. W. Determination of the Ionization Energy of the Benzene-Argon Complex by Zero Kinetic Energy Photoelectron Spectroscopy. Chem. Phys. Lett. 1987, 135, 219-222.

(221) Koch, H.; Fernández, B.; Makarewicz, J. Ground State Benzene-Argon Intermolecular Potential Energy Surface. J. Chem. Phys. 1999, 111, 198-204.

(222) Klopper, W.; Lüthi, H. P.; Brupbacher, T.; Bauder, A. Ab initio Computations close to the One-particle Basis Set Limit on the weakly bound van der Waals Complexes Benzene-Neon and Benzene-Argon. J. Chem. Phys. 1994, 101, 9747-9754.

(223) Peterson, K. A.; Adler, T. B.; Werner, H.-J. Systematically Convergent Basis Sets for Explicitly Correlated Wavefunctions: The atoms H, He, B-Ne, and Al-Ar. J. Chem. Phys. 2008, $128,084102$. 
(224) Yousaf, K. E.; Peterson, K. A. Optimized Auxiliary Basis Sets for Explicitly Correlated Methods. J. Chem. Phys. 2008, 129, 184108.

(225) Hill, J. G.; Peterson, K. A. Correlation Consistent Basis Sets for Explicitly Correlated Wavefunctions: Pseudopotential-based Basis Sets for the post-d Main Group Elements Ga - Rn. J. Chem. Phys. 2014, 141, 094106.

(226) Bachorz, R. A.; Bischoff, F. A.; Glöß, A.; Hättig, C.; Höfener, S.; Klopper, W.; Tew, D. P. The MP2-F12 Method in the TURBOMOLE Program Package. J. Comput. Chem. 2011, 32, $2492-2513$.

(227) MOLPRO, version 2012.1, a Package of Ab Initio Programs, H.-J. Werner, P. J. Knowles, G. Knizia, F. R. Manby, M. Schütz, P. Celani, T. Korona, R. Lindh, A. Mitrushenkov, G. Rauhut, K. R. Shamasundar, T. B. Adler, R. D. Amos, A. Bernhardsson, A. Berning, D. L. Cooper, M. J. O. Deegan, A. J. Dobbyn, F. Eckert, E. Goll, C. Hampel, A. Hesselmann, G. Hetzer, T. Hrenar, G. Jansen, C. Köppl, Y. Liu, A. W. Lloyd, R. A. Mata, A. J. May, S. J. McNicholas, W. Meyer, M. E. Mura, A. Nicklass, D. P. O’Neill, P. Palmieri, D. Peng, K. Pflüger, R. Pitzer, M. Reiher, T. Shiozaki, H. Stoll, A. J. Stone, R. Tarroni, T. Thorsteinsson, and M. Wang, see http: //www. molpro.net.

(228) Werner, H.-J.; Knowles, P. J.; Knizia, G.; Manby, F. R.; Schütz, M. Molpro: a GeneralPurpose Quantum Chemistry Program Package. WIREs: Comput. Mol. Sci. 2012, 2, 242253.

(229) Perdew, J. P.; Burke, K.; Ernzerhof, M. Generalized Gradient Approximation Made Simple. Phys. Rev. Lett. 1996, 77, 3865-3868.

(230) Weigend, F.; Ahlrichs, R. Balanced Basis Sets of Split Valence, Triple Zeta Valence and Quadruple Zeta Valence Quality for H to Rn: Design and Assessment of Accuracy. Phys. Chem. Chem. Phys. 2005, 7, 3297-3305. 
(231) Steed, J. M.; Dixon, T. A.; Klemperer, W. Molecular-Beam Studies of Benzene Dimer, Hexafluorobenzene Dimer and Benzene-Hexafluorobenzene. J. Chem. Phys. 1979, 70, 49404946.

(232) Arunan, E.; Gutowsky, H. S. The Rotational Spectrum, Structure and Dynamics of a Benzene Dimer. J. Chem. Phys. 1993, 98, 4294-4296.

(233) Podeszwa, R.; Bukowski, R.; Szalewicz, K. Potential Energy Surface for the Benzene Dimer and Perturbational Analysis of pi-pi Interactions. J. Phys. Chem. A 2006, 110, 10345-10354.

(234) van der Avoird, A.; Podeszwa, R.; Szalewicz, K.; Leforestier, C.; van Harrevelt, R.; Bunker, P. R.; Schnell, M.; von Helden, G.; Meijer, G. Vibration-Rotation-Tunneling States of the Benzene Dimer: An Ab Initio Study. Phys. Chem. Chem. Phys. 2010, 12, 8219-8240.

(235) Schnell, M.; Erlekam, U.; Bunker, P. R.; von Helden, G.; Grabow, J.-U.; Meijer, G.; van der Avoird, A. Structure of the Benzene Dimer - Governed by Dynamics. Angew. Chem. Int. Ed. 2013, 52, 5180-5183.

(236) Schnell, M.; Erlekam, U.; Bunker, P. R.; von Helden, G.; Grabow, J.-U.; Meijer, G.; van der Avoird, A. Unraveling the Internal Dynamics of the Benzene Dimer: A Combined Theoretical and Microwave Spectroscopy Study. Phys. Chem. Chem. Phys. 2013, 15, 10207-10223.

(237) Grover, J. R.; Walters, E. A.; Hui, E. T. Dissociation Energies of the Benzene Dimer and Dimer Cation. J. Phys. Chem. 1987, 91, 3233-3237.

(238) Krause, H.; Ernstberger, B.; Neusser, H. Binding Energies of small Benzene Clusters. Chem. Phys. Lett. 1991, 184, 411-417.

(239) Nishiyama, I.; Hanazaki, I. Infrared Photodissociation of the Benzene Dimer. Translational Energy Distribution of Dissociation Fragments. Chem. Phys. Lett. 1985, 117, 99-102. 
(240) Chewter, L. A.; Sander, M.; Müller-Dethlefs, K.; Schlag, E. W. High Resolution Zero Kinetic Energy Photoelectron Spectroscopy of Benzene and Determination of the Ionization Potential. J. Chem. Phys. 1987, 86, 4737-4744.

(241) Nemeth, G. I.; Selzle, H. L.; Schlag, E. W. Magnetic \{ZEKE\} Experiments with Mass Analysis. Chem. Phys. Lett. 1993, 215, 151-155.

(242) Boernsen, K. O.; Selzle, H. L.; Schlag, E. W. Photoionization of Benzene Complexes. J. Phys. Chem. 1988, 92, 5482-5485.

(243) Grover, J. R.; Walters, E. A.; Baumgaertel, H. Comparison of One-photon with Two-photon Photoionization of Benzene Dimer near Threshold: Comment. J. Phys. Chem. 1989, 93, $7534-7535$.

(244) Selzle, H. L.; Neusser, H. J.; Ernstberger, B.; Krause, H.; Schlag, E. W. Ionization Potential of the Benzene Dimer: Reply to Comment. J. Phys. Chem. 1989, 93, 7535-7536.

(245) Pieniazek, P. A.; Krylov, A. I.; Bradforth, S. E. Electronic Structure of the Benzene Dimer Cation. J. Chem. Phys. 2007, 127, 044317.

(246) Johnson, R. D.; Burdenski, S.; Hoffbauer, M. A.; Giese, C. F.; Gentry, W. R. Infrared Photodissociation of Benzene Dimers in the $1000 \mathrm{~cm}^{-1}$ Frequency Region. J. Chem. Phys. 1986, 84, 2624-2629.

(247) Henson, B. F.; Hartland, G. V.; Venturo, V. A.; Felker, P. M. Time-resolved Raman spectroscopy of Benzene Dimer: Nanosecond-plus Lifetime at $992 \mathrm{~cm}^{-1}$ Vibrational Energy. $J$. Chem. Phys. 1989, 91, 2751-2753.

(248) Tsuzuki, S.; Honda, K.; Uchimaru, T.; Mikami, M. Ab Initio Calculations of Structures and Interaction Eenergies of Toluene Dimers Including CCSD(T) Level Electron Correlation correction. J. Chem. Phys. 2005, 122, 144323. 
(249) Chipot, C.; Jaffe, R.; Maigret, B.; Pearlman, D. A.; Kollman, P. A. Benzene Dimer:âĂL' A Good Model for pi-pi Interactions in Proteins? A Comparison between the Benzene and the Toluene Dimers in the Gas Phase and in an Aqueous Solution. J. Am. Chem. Soc. 1996, 118, $11217-11224$.

(250) Rogers, D. M.; Hirst, J. D.; Lee, E. P.; Wright, T. G. Ab Initio Study of the Toluene Dimer. Chem. Phys. Lett. 2006, 427, 410-413.

(251) Law, K.; Schauer, M.; Bernstein, E. R. Dimers of Aromatic Molecules: (Benzene) ${ }_{2}$, (Toluene) $)_{2}$, and Benzene-Toluene. J. Chem. Phys. 1984, 81, 4871-4882.

(252) Ishikawa, S.; Ebata, T.; Ishikawa, H.; Inoue, T.; Mikami, N. Hole-Burning and Stimulated RamanâĹ̌̌UV Double Resonance Spectroscopies of Jet-Cooled Toluene Dimer. J. Phys. Chem. 1996, 100, 10531-10535.

(253) Geng, Y.; Takatani, T.; Hohenstein, E. G.; Sherrill, C. D. Accurately Characterizing the pipi Interaction Energies of Indole-Benzene Complexes. J. Phys. Chem. A 2010, 114, 35763582.

(254) Biswal, H. S.; Gloaguen, E.; Mons, M.; Bhattacharyya, S.; Shirhatti, P. R.; Wategaonkar, S. Structure of the Indole-Benzene Dimer Revisited. J. Phys. Chem. A 2011, 115, 9485-9492.

(255) Lahmani, F.; Lardeux-Dedonder, C.; Solgadi, D.; Zehnacker, A. Spectroscopic Study of the Anisole-Benzene Complex Formed in a Supersonic Free Jet. J. Phys. Chem. 1989, 93, 3984-3989.

(256) Reimann, B.; Buchhold, K.; Barth, H.-D.; Brutschy, B.; Tarakeshwar, P.; Kim, K. S. Anisole- $\left(\mathrm{H}_{2} \mathrm{O}\right)_{n}(\mathrm{n}=1-3)$ Complexes: An Experimental and Theoretical Investigation of the Modulation of Optimal Structures, Binding Energies, and Vibrational Spectra in Both the Ground and First Excited States. J. Chem. Phys. 2002, 117, 8805-8822. 
(257) Ribblett, J. W.; Sinclair, W. E.; Borst, D. R.; Yi, J. T.; Pratt, D. W. High Resolution Electronic Spectra of Anisole and Anisole-Water in the Gas Phase: Hydrogen Bond Switching in the $S_{1}$ State. J. Phys. Chem. A 2006, 110, 1478-1483.

(258) Biczysko, M.; Piani, G.; Pasquini, M.; Schiccheri, N.; Pietraperzia, G.; Becucci, M.; Pavone, M.; Barone, V. On the Properties of Microsolvated Molecules in the Ground (S0) and Excited (S1) States: The Anisole-Ammonia 1:1 Complex. J. Chem. Phys. 2007, 127, 144303.

(259) Heger, M.; Altnoder, J.; Poblotzki, A.; Suhm, M. A. To pi or Not to pi - How does Methanol Dock onto Anisole? Phys. Chem. Chem. Phys. 2015, 17, 13045-13052.

(260) Giuliano, B. M.; Maris, A.; Melandri, S.; Caminati, W. Pure Rotational Spectrum and Model Calculations of Anisole-Ammonia. J. Phys. Chem. A 2009, 113, 14277-14280.

(261) Pietraperzia, G.; Pasquini, M.; Schiccheri, N.; Piani, G.; Becucci, M.; Castellucci, E.; Biczysko, M.; Bloino, J.; Barone, V. The Gas Phase Anisole Dimer: A Combined HighResolution Spectroscopy and Computational Study of a Stacked Molecular System. J. Phys. Chem. A 2009, 113, 14343-14351.

(262) Pasquini, M.; G.Pietraperzia,; Piani, G.; Becucci, M. Excitonic Coupling in van der Waals Complexes: The Anisole Dimers. J. Mol. Struct. 2011, 993, 491-494.

(263) Balmer, F. A.; Trachsel, M. A.; van der Avoird, A.; Leutwyler, S. The Elusive $S_{2}$ State, the $S_{1} / S_{2}$ Splitting, and the Excimer States of the Benzene Dimer. J. Chem. Phys. 2015, 142, 234306.

(264) Wei, J.; Makarem, C.; Reinitz, A. L.; Darr, J. P.; Loomis, R. A. Accurate Measurement of the T-Shaped and Linear Binding Energies Using Vibronic-Specific $\mathrm{I}_{2}(\mathrm{~B}, \mathrm{v})$ Fragment Velocity-Map Imaging. Chem. Phys. 2012, 399, 172-179. 
(265) Mollner, A. K.; Casterline, B. E.; Ch' ng, L. C.; Reisler, H. Imaging the State-Specific Vibrational Predissociation of the Ammonia - Water Hydrogen-Bonded Dimer. J. Phys. Chem. A 2009, 113, 10174-10183.

(266) Lane, J. R.; Vaida, V.; Kjaergaard, H. G. Calculated Electronic Transitions of the Water Ammonia Complex. J. Chem. Phys. 2008, 128, 034302.

(267) Slipchenko, M. N.; Sartakov, B. G.; Vilesov, A. F.; Xantheas, S. S. Study of NH Stretching Vibrations in Small Ammonia Clusters by Infrared Spectroscopy in He Droplets and ab Initio Calculations. J. Phys. Chem. A 2007, 111, 7460-7471.

(268) Ni, H.; Serafin, J. M.; Valentini, J. J. Dynamics of the Vibrational Predissociation of HCl Dimer. J. Chem. Phys. 2000, 113, 3055-3066.

(269) Vissers, G. W. M.; Oudejans, L.; Miller, R. E.; Groenenboom, G. C.; van der Avoird, A. Vibrational Predissociation in the HCl Dimer. J. Chem. Phys. 2004, 120, 9487-9498.

(270) Mancini, J. S.; Bowman, J. M. A New Many-Body Potential Energy Surface for HCl Clusters and Its Application to Anharmonic Spectroscopy and Vibration-Vibration Energy Transfer in the $\mathrm{HCl}$ Trimer. J. Phys. Chem. A 2014, 118, 7367-7374.

(271) Bohac, E. J.; Marshall, M. D.; Miller, R. E. Initial State Effects in the Vibrational Predissociation of Hydrogenfluoride Dimer. J. Chem. Phys. 1992, 96, 6681-6695.

(272) Klopper, W.; Quack, M.; Suhm, M. A. HF Dimer: Empirically Refined Analytical Potential Energy and Dipole Hypersurfaces from Ab Initio Calculations. J. Chem. Phys. 1998, 108, 10096-10115.

(273) Oudejans, L.; Miller, R. E. Dissociation Dynamics of Oriented DF-HF and HF-DF Complexes: Evidence for Direct and Indirect Dissociation. J. Phys. Chem. A 1997, 101, 75827592. 
(274) Klopper, W.; Quack, M.; Suhm, M. A. A New Ab Initio Based Six-dimensional Semiempirical Pair Interaction Potential for HF. Chem. Phys. Lett. 1996, 261, 35-44.

(275) Oudejans, L.; Moore, D. T.; Miller, R. E. State-to-State Vibrational Predissociation Dynamics of the Acetylene-HF complex. J. Chem. Phys. 1999, 110, 209-219.

(276) Oudejans, L.; Miller, R. E. State-to-State Vibrational Predissociation Dynamics of the Acetylene-HCl Complex. J. Phys. Chem. A 1999, 103, 4791-4797.

(277) Li, G.; Parr, J.; Fedorov, I.; Reisler, H. Imaging Study of Vibrational Predissociation of the HCl-Acetylene Dimer: Pair-correlated Distributions. Phys. Chem. Chem. Phys. 2006, 8, 2915-2924.

(278) Parr, J. A.; Li, G.; Fedorov, I.; McCaffery, A. J.; Reisler, H. Imaging the State-Specific Vibrational Predissociation of the $\mathrm{C}_{2} \mathrm{H}_{2}-\mathrm{NH}_{3}$ Hydrogen-Bonded Dimer. J. Phys. Chem. A 2007, 111, 7589-7598.

(279) Bohac, E. J.; Miller, R. E. State-to-state Vibrational Predissociation of $\mathrm{H}_{2}-\mathrm{HF}$ and $\mathrm{D}_{2}-\mathrm{HF}$. Direct Comparisons between Theory and Experiment. J. Chem. Phys. 1993, 98, 2604-2613.

(280) Bemish, R. J.; Bohac, E. J.; Wu, M.; Miller, R. E. Photofragment Vibrational, Rotational, and Translational Distributions for $\mathrm{N}_{2}-\mathrm{HF}$ (v=1). J. Chem. Phys. 1994, 101, 9457-9468.

(281) Jankowski, P.; Tsang, S. N.; Klemperer, W.; Szalewicz, K. Spectra of $\mathrm{N}_{2}-\mathrm{HF}$ from Symmetry-adapted Perturbation Theory Potential. J. Chem. Phys. 2001, 114, 8948-8963.

(282) Oudejans, L.; Miller, R. E. Intermolecular V-V Energy Transfer in the Photodissociation of $\mathrm{CO}_{2}-\mathrm{HF}(\mathrm{v}=1)$. J. Chem. Phys. 1998, 109, 3474-3484.

(283) Oudejans, L.; Miller, R. Mode Dependence of the State-to-State Vibrational Dynamics of HCN-HF. Chem. Phys. 1998, 239, 345 - 356.

(284) Shorter, J. H.; Casassa, M. P.; King, D. S. Fragment State Correlations in the Dissociation of NO-HF(v=1). J. Chem. Phys. 1992, 97, 1824-1831. 
(285) Hetzler, J. R.; Casassa, M. P.; King, D. S. Product Energy Correlations in the Dissociation of Overtone Excited Nitric Oxide Dimer. J. Phys. Chem. 1991, 95, 8086-8095.

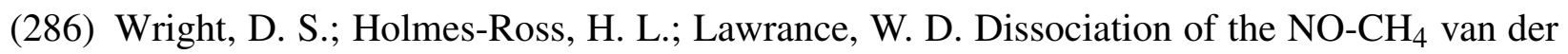
Waals Complex: Binding Energy and Correlated Motion of the Molecular Fragments. Chem. Phys. Lett. 2007, 435, 19-23.

(287) Krause, H.; Neusser, H. J. The van der Waals modes of the (Benzene-Ar) ${ }^{+}$Complex Studied by Mass-selective Pulsed-field Threshold Ionization. Chem. Phys. Lett. 1993, 213, 603-609.

(288) Hobza, P.; Bludsky, O.; Selzle, H. L.; Schlag, E. W. Ab-Initio Second- and Fourthorder Moller-Plesset Study on Structure, Stabilization Energy, and Stretching Vibration of Benzene-X (X=He, Ne, Ar, Kr, Xe) van der Waals molecules. J. Chem. Phys. 1992, 97, $335-340$.

(289) Makarewicz, J. Fully Dimensional Ab Initio Description of the Structure and Energetics of Azabenzene-Argon Complexes. J. Chem. Phys. 2005, 123, 154302.

(290) Cagide Fajín, J. L.; López Cacheiro, J.; Fernández, B.; Makarewicz, J. Fluorobenzene-Argon Ground-state Intermolecular Potential Energy Surface. J. Chem. Phys. 2004, 120, 8582-8586.

(291) Su, M.-C.; O, H.-K.; Parmenter, C. S. A Fluorescence Characterization of the pDifluorobenzene-Argon van der Waals Complex. Energy Levels, Geometries and Dissociation Energies. Chem. Phys. 1991, 156, 261-280.

(292) Makarewicz, J. Potential-energy Surface, Dynamics of van der Waals Motions, and Vibronic Transitions in p-Difluorobenzene-Argon Complex. J. Chem. Phys. 2005, 122, 114312.

(293) Dimopoulou-Rademann, O.; Even, U.; Amirav, A.; Jortner, J. Binding energy of the Styrene-Argon van der Waals Molecule. J. Phys. Chem. 1988, 92, 5371-5373. 
(294) Dao, P.; Morgan, S.; Jr., A. C. Two-color Resonance Enhanced Multiphoton Ionization of van der Waals Molecules: Studies of Spectroscopy Shifts and Ionization Thresholds of pXylene Clustered with Argon. Chem. Phys. Lett. 1985, 113, 219-224.

(295) Černý, J.; Tong, X.; Hobza, P.; Müller-Dethlefs, K. State of the Art Theoretical Study and Comparison to Experiment for the Phenol-..Argon Complex. J. Chem. Phys. 2008, 128, 114319.

(296) Makarewicz, J. Potential Energy Surface, van der Waals Motions, and Vibronic Transitions in Phenol-Argon Complex. J. Chem. Phys. 2006, 124, 084310.

(297) Georgiev, S.; Chakraborty, T.; Neusser, H. J. Binding Energy and Intermolecular Vibrations of Neutral and Ionized p-Fluorotoluene-Ar Cluster by Mass Analyzed Threshold Ionization. J. Phys. Chem. A 2004, 108, 3304-3307.

(298) Mazzoni, F.; Becucci, M.; Rezac, J.; Nachtigallová, D.; Michels, F.; Hobza, P.; MüllerDethlefs, K. Structure and Energetics of the Anisole-Ar $\operatorname{Ar}_{n}(\mathrm{n}=1,2,3)$ Complexes: Highresolution Resonant Two-photon and Threshold Ionization Experiments, and Quantum Chemical Calculations. Phys. Chem. Chem. Phys. 2015, 12530-12537.

(299) Outhouse, E. A.; Bickel, G. A.; Demmer, D. R.; Wallace, S. C. Vibrational Predissociation in $S_{1}$ Indole van der Waals Clusters. J. Chem. Phys. 1991, 95, 6261-6270.

(300) Outhouse, E. A.; Demmer, D. R.; Leach, G. W.; Wallace, S. C. Vibrational Predissociation in $\mathrm{S}_{1}$ 1-Methylindole van der Waals Clusters. J. Chem. Phys. 1993, 99, 80-90.

(301) Grebner, T. L.; Neusser, H. J. Pulsed Field Threshold Ionization of van der Waals Complexes. The Dissociation Energy of Ionic and Neutral Dibenzo-p-dioxin·Ar and -84Kr. Chem. Phys. Lett. 1995, 245, 578-584.

(302) Gaber, A.; Riese, M.; Witte, F.; Grotemeyer, J. Determination of the Binding Energies in Aromatic Clusters: Resonance-enhanced Multi-photon Ionization and Mass Analyzed 
Threshold Ionization Investigation of the Dichlorobenzene-Argon Complexes. Eur. J. Mass Spectrom. 2009, 15, 349-359.

(303) Gilbert, B. D.; Parmenter, C. S.; Oh, H.-K. Vibrational Predissociation Dynamics of pDifluorobenzene- $\mathrm{N}_{2}$ Complexes. Comparison with p-Difluorobenzene-Ar. J. Phys. Chem. 1995, 99, 2444-2458.

(304) Fujiwara, T.; Lim, E. C. Binding Energies of the Neutral and Ionic Clusters of Naphthalene in Their Ground Electronic States. J. Phys. Chem. A 2003, 107, 4381-4386.

(305) Podeszwa, R.; Szalewicz, K. Physical Origins of Interactions in Dimers of Polycyclic Aromatic Hydrocarbons. Phys. Chem. Chem. Phys. 2008, 10, 2735-2746. 